\title{
Cochrane
}

Library

Cochrane Database of Systematic Reviews

\section{Interventions for treating urinary incontinence after stroke in adults} (Review)

Thomas LH, Coupe J, Cross LD, Tan AL, Watkins CL

Thomas LH, Coupe J, Cross LD, Tan AL, Watkins CL.

Interventions for treating urinary incontinence after stroke in adults.

Cochrane Database of Systematic Reviews 2019, Issue 2. Art. No.: CD004462.

DOI: 10.1002/14651858.CD004462.pub4.

www.cochranelibrary.com 
TABLE OF CONTENTS

ABSTRAC

PLAIN LANGUAGE SUMMARY

SUMMARY OF FINDINGS

BACKGROUND

OBJECTIVES

METHODS

RESULTS

Figure 1.

Figure 2.

Figure 3.

DISCUSSION

AUTHORS' CONCLUSIONS

ACKNOWLEDGEMENTS

REFERENCES

CHARACTERISTICS OF STUDIES

DATA AND ANALYSES

Analysis 1.1. Comparison 1 Intervention versus no intervention/usual care, Outcome 1 Number of participants continent after treatment.

Analysis 1.2. Comparison 1 Intervention versus no intervention/usual care, Outcome 2 Number of incontinent episodes in 24 hours (mean).

Analysis 1.3. Comparison 1 Intervention versus no intervention/usual care, Outcome 3 Number of participants cured of all four urinary symptoms.

Analysis 1.4. Comparison 1 Intervention versus no intervention/usual care, Outcome 4 Urinary symptoms - frequency (continuous variables).

Analysis 1.5. Comparison 1 Intervention versus no intervention/usual care, Outcome 5 Urinary symptoms - frequency (dichotomous variables).

Analysis 1.6. Comparison 1 Intervention versus no intervention/usual care, Outcome 6 Urinary symptoms - urgency. ............ Analysis 1.7. Comparison 1 Intervention versus no intervention/usual care, Outcome 7 Urinary symptoms - nocturia (continuous variables).

Analysis 1.8. Comparison 1 Intervention versus no intervention/usual care, Outcome 8 Urinary symptoms - nocturia (dichotomous variables).

Analysis 1.9. Comparison 1 Intervention versus no intervention/usual care, Outcome 9 Physical measures: mean function of the pelvic floor muscle.

Analysis 1.10. Comparison 1 Intervention versus no intervention/usual care, Outcome 10 Health status and quality of life health status, mean total score 36-Item Short Form.

Analysis 1.11. Comparison 1 Intervention versus no intervention/usual care, Outcome 11 Health status and quality of life quality of life.

Analysis 1.12. Comparison 1 Intervention versus no intervention/usual care, Outcome 12 Functional ability - mean Barthel score (continuous variables)

Analysis 1.13. Comparison 1 Intervention versus no intervention/usual care, Outcome 13 Participant satisfaction - numbers who were dissatisfied.

Analysis 2.1. Comparison 2 Intervention versus placebo, Outcome 1 Number of participants continent after treatment. .......... Analysis 2.2. Comparison 2 Intervention versus placebo, Outcome 2 Number of incontinent episodes - mean per day. ........... Analysis 2.3. Comparison 2 Intervention versus placebo, Outcome 3 Number of incontinent episodes - mean per week. ......... Analysis 2.4. Comparison 2 Intervention versus placebo, Outcome 4 Health status and quality of life - quality of life. .............. Analysis 4.1. Comparison 4 Combined intervention versus single intervention, Outcome 1 Number of participants continent after treatment.

Analysis 4.2. Comparison 4 Combined intervention versus single intervention, Outcome 2 Number of incontinent episodes mean.

Analysis 5.1. Comparison 5 Specific intervention versus attention control, Outcome 1 Number of participants continent after treatment. 
[Intervention Review]

\section{Interventions for treating urinary incontinence after stroke in adults}

Lois H Thomas ${ }^{1}$, Jacqueline Coupe ${ }^{1}$, Lucy D Cross², Aidan L Tan ${ }^{3}$, Caroline L Watkins ${ }^{1}$

1Faculty of Health and Wellbeing, University of Central Lancashire, Preston, UK. ${ }^{2}$ Research Support Team, Faculty of Health and Wellbeing, University of Central Lancashire, Preston, UK. ${ }^{3}$ Department of Preventive Medicine, National University Hospital, 5 Lower Kent Ridge Road, Singapore

Contact: Lois H Thomas, Faculty of Health and Wellbeing, University of Central Lancashire, Room 416, Brook Building, Preston, Lancashire, PR1 2HE, UK. Ihthomas@uclan.ac.uk.

Editorial group: Cochrane Incontinence Group.

Publication status and date: New search for studies and content updated (no change to conclusions), published in Issue 2, 2019.

Citation: Thomas LH, Coupe J, Cross LD, Tan AL, Watkins CL. Interventions for treating urinary incontinence after stroke in adults. Cochrane Database of Systematic Reviews 2019, Issue 2. Art. No.: CD004462. DOI: 10.1002/14651858.CD004462.pub4.

Copyright ( 2019 The Cochrane Collaboration. Published by John Wiley \& Sons, Ltd.

\section{A B S T R A C T}

\section{Background}

Urinary incontinence can affect $40 \%$ to $60 \%$ of people admitted to hospital after a stroke, with $25 \%$ still having problems when discharged from hospital and $15 \%$ remaining incontinent after one year.

This is an update of a review published in 2005 and updated in 2008.

\section{Objectives}

To assess the effects of interventions for treating urinary incontinence after stroke in adults at least one-month post-stroke.

\section{Search methods}

We searched the Cochrane Incontinence and Cochrane Stroke Specialised Registers (searched 30 October 2017 and 1 November 2017 respectively), which contain trials identified from the Cochrane Central Register of Controlled Trials (CENTRAL), MEDLINE, MEDLINE InProcess, MEDLINE Epub Ahead of Print, CINAHL, ClinicalTrials.gov, WHO ICTRP and handsearched journals and conference proceedings.

\section{Selection criteria}

We included randomised or quasi-randomised controlled trials.

\section{Data collection and analysis}

Two review authors independently undertook data extraction, risk of bias assessment and implemented GRADE.

\section{Main results}

We included 20 trials (reporting 21 comparisons) with 1338 participants. Data for prespecified outcomes were not available except where reported below.

\section{Intervention versus no intervention/usual care}

Behavioural interventions: Low-quality evidence suggests behavioural interventions may reduce the mean number of incontinent episodes in 24 hours (mean difference (MD) -1.00, 95\% confidence interval $(\mathrm{Cl})-2.74$ to $0.74 ; 1$ trial; 18 participants; $P=0.26)$. Further, low-quality evidence from two trials suggests that behavioural interventions may make little or no difference to quality of life (SMD $-0.99,95 \% \mathrm{Cl}-2.83$ to $0.86 ; 55$ participants). 
Specialised professional input interventions: One trial of moderate-quality suggested structured assessment and management by continence nurse practitioners probably made little or no difference to the number of people continent three months after treatment (risk ratio (RR) $1.28,95 \% \mathrm{Cl} 0.81$ to $2.02 ; 121$ participants; equivalent to an increase from 354 to 453 per $1000,95 \% \mathrm{Cl} 287$ to 715 ).

Complementary therapy: Five trials assessed complementary therapy using traditional acupuncture, electroacupuncture and ginger-saltpartitioned moxibustion plus routine acupuncture. Low-quality evidence from five trials suggested that complementary therapy may increase the number of participants continent after treatment; participants in the treatment group were three times more likely to be continent (RR 2.82, 95\% Cl 1.57 to 5.07; 524 participants; equivalent to an increase from 193 to 544 per 1000, 95\% Cl 303 to 978). Adverse events were reported narratively in one study of electroacupuncture, reporting on bruising and postacupuncture abdominal pain in the intervention group.

Physical therapy: Two trials reporting three comparisons suggest that physical therapy using transcutaneous electrical nerve stimulation (TENS) may reduce the mean number of incontinent episodes in 24 hours (MD $-4.76,95 \% \mathrm{Cl}-8.10$ to -1.41 ; 142 participants; low-quality evidence). One trial of TENS reporting two comparisons found that the intervention probably improves overall functional ability (MD 8.97, $95 \% \mathrm{Cl} 1.27$ to 16.68 ; 81 participants; moderate-quality evidence).

\section{Intervention versus placebo}

Physical therapy: One trial of physical therapy suggests TPTNS may make little or no difference to the number of participants continent after treatment (RR $0.75,95 \% \mathrm{Cl} 0.19$ to $3.04 ; 54$ participants) or number of incontinent episodes (MD $-1.10,95 \% \mathrm{Cl}-3.99$ to $1.79 ; 39$ participants). One trial suggested improvement in the TPTNS group at 26 -weeks (OR $0.04,95 \% \mathrm{Cl} 0.004$ to 0.41 ) but there was no evidence of a difference in perceived bladder condition at six weeks (OR 2.33, $95 \% \mathrm{Cl} 0.63$ to 8.65 ) or 12 weeks (OR $1.22,95 \% \mathrm{Cl} 0.29$ to 5.17 ). Data from one trial provided no evidence that TPTNS made a difference to quality of life measured with the ICIQLUTSqol (MD 3.90, 95\% Cl -4.25 to $12.05 ; 30$ participants). Minor adverse events, such as minor skin irritation and ankle cramping, were reported in one study.

Pharmacotherapy interventions: There was no evidence from one study that oestrogen therapy made a difference to the mean number of incontinent episodes per week in mild incontinence (paired samples, MD $-1.71,95 \% \mathrm{Cl}-3.51$ to 0.09 ) or severe incontinence (paired samples, $\mathrm{MD}-6.40,95 \% \mathrm{Cl}-9.47$ to -3.33$)$. One study reported no adverse events.

\section{Specific intervention versus another intervention}

Behavioural interventions: One trial comparing a behavioural intervention (timed voiding) with a pharmacotherapy intervention (oxybutynin) contained no useable data.

Complementary therapy: One trial comparing different acupuncture needles and depth of needle insertion to assess the effect on incontinence reported that, after four courses of treatment, $78.1 \%$ participants in the elongated needle group had no incontinent episodes versus $40 \%$ in the filiform needle group (57 participants). This trial was assessed as unclear or high for all types of bias apart from incomplete outcome data.

\section{Combined intervention versus single intervention}

One trial compared a combined intervention (sensory motor biofeedback plus timed prompted voiding) against a single intervention (timed voiding). The combined intervention may make little or no difference to the number of participants continent after treatment (RR $0.55,95 \% \mathrm{Cl} 0.06$ to $5.21 ; 23$ participants; equivalent to a decrease from 167 to 92 per $1000,95 \% \mathrm{Cl} 10$ to 868 ) or to the number of incontinent episodes (MD 2.20, 95\% Cl 0.12 to $4.28 ; 23$ participants).

\section{Specific intervention versus attention control}

Physical therapy interventions: One study found TPTNS may make little or no difference to the number of participants continent after treatment compared to an attention control group undertaking stretching exercises (RR 1.33, 95\% Cl 0.38 to 4.72; 24 participants; equivalent to an increase from 250 to 333 per 1000, 95\% Cl 95 to 1000).

\section{Authors' conclusions}

There is insufficient evidence to guide continence care of adults in the rehabilitative phase after stroke. As few trials tested the same intervention, conclusions are drawn from few, usually small, trials. Cls were wide, making it difficult to ascertain if there were clinically important differences. Only four trials had adequate allocation concealment and many were limited by poor reporting, making it impossible to judge the extent to which they were prone to bias. More appropriately powered, multicentre trials of interventions are required to provide robust evidence for interventions to improve urinary incontinence after stroke.

\section{PLAIN LANGUAGE SUMMARY}

\section{Treatments for urinary incontinence after stroke in adults}

\section{Review question}


We wanted to assess the effectiveness of interventions aimed at helping urinary incontinence in adults who had a stroke more than one month before.

\section{Background}

Half of people admitted to hospital with a stroke have urinary incontinence. As well as involuntary loss of urine, symptoms of urinary incontinence include having an urgent desire to pass urine (urge incontinence) or leaking urine when laughing or sneezing (stress incontinence). These symptoms are more severe in stroke survivors than in other people with urinary incontinence. They cause embarrassment and distress and affect people's ability to take part in rehabilitation. Urinary incontinence reduces feelings of self-worth and depression is common. It also has a major impact on families and may affect whether patients are able to return home.

\section{Search date}

The search is current to 1 November 2017.

\section{Study characteristics}

We identified 20 studies identifying 21 comparisons and involving 1338 people. These studies included a variety of behavioural therapies (e.g. pelvic floor muscle training), complementary therapies (e.g. manual acupuncture or electroacupuncture) and physical therapies (e.g. transcutaneous electrical nerve stimulation, TENS), as well as medicines (e.g. oxybutynin, oestrogen). One trial investigated the effect of assessment and treatment by a continence nurse practitioner. Control groups were generally 'usual care' or no treatment.

\section{Key results}

We found that behavioural interventions may reduce the mean number of incontinent episodes in 24 hours but may make little or no difference to quality of life. However, intervention from a continence nurse practitioner probably made little or no difference to the number of people continent three months after treatment. Complementary therapies such as acupuncture may increase the number of participants continent after treatment. Physical therapies, such as transcutaneous electrical nerve stimulation, may reduce the average number of incontinent episodes in 24 hours and probably improves functional ability.

\section{Quality of the evidence}

The quality of the evidence was limited due to poor reporting of study details (particularly in the earlier studies) and the small number of study participants in most comparisons. More than half of the studies did not provide information on side effects.

\section{Authors' conclusions}

High-quality trials comparing different treatments to usual care or no treatment involving larger numbers of participants are needed. 
SUMMARY OF FINDINGS

Summary of findings for the main comparison. Behavioural interventions compared with usual care or no treatment for treating urinary incontinence after stroke

Behavioural interventions compared with usual care or no treatment for treating urinary incontinence after stroke

Patient or population: people with stroke and urinary incontinence

Settings: hospital, clinic or home

Intervention: behavioural interventions

Comparison: no treatment/usual care

\begin{tabular}{|c|c|c|c|c|c|c|}
\hline \multirow[t]{3}{*}{ Outcomes } & \multicolumn{2}{|c|}{ Illustrative comparative risks* $(95 \% \mathrm{CI})$} & \multirow{3}{*}{$\begin{array}{l}\text { Relative effect } \\
(95 \% \mathrm{CI})\end{array}$} & \multirow{3}{*}{$\begin{array}{l}\text { No of partici- } \\
\text { pants } \\
\text { (studies) }\end{array}$} & \multirow{3}{*}{$\begin{array}{l}\text { Quality of the } \\
\text { evidence } \\
\text { (GRADE) }\end{array}$} & \multirow[t]{3}{*}{ Comments } \\
\hline & Assumed risk & $\begin{array}{l}\text { Corresponding } \\
\text { risk }\end{array}$ & & & & \\
\hline & Control & Intervention & & & & \\
\hline $\begin{array}{l}\text { Number of participants continent af- } \\
\text { ter treatment }\end{array}$ & \multicolumn{2}{|l|}{ Study population } & - & - & - & Not reported \\
\hline Number of incontinent episodes & $\begin{array}{l}\text { The mean num- } \\
\text { ber of incontinent } \\
\text { episodes in the } \\
\text { control group was } \\
1.2\end{array}$ & $\begin{array}{l}\text { The mean num- } \\
\text { ber of incontinent } \\
\text { episodes in the in- } \\
\text { tervention group } \\
\text { was } 0.2\end{array}$ & $\begin{array}{l}\text { MD -1.00 } \\
(-2.74 \text { to } 0.74)\end{array}$ & $\begin{array}{l}18 \text { participants } \\
\text { (1) }\end{array}$ & $\begin{array}{l}\oplus \oplus \odot \odot \\
\text { Low }^{a}\end{array}$ & $\begin{array}{l}\text { Outcome reported de- } \\
\text { scriptively for Tibaek } \\
2017 \text { : the reported num- } \\
\text { ber of Ul episodes per } 24 \\
\text { hours was very small (in- } \\
\text { tervention } 0 \text { at post-test } \\
\text { and } 1 \text { at follow-up; con- } \\
\text { trol } 0 \text { at post-test, } 0 \text { at } \\
\text { follow-up). }\end{array}$ \\
\hline Perception of improvement or cure & - & - & - & - & - & Not reported \\
\hline $\begin{array}{l}\text { Health status and quality of life } \\
\text { assessed by Bristol Female Lower Uri- } \\
\text { nary Tract Symptoms Questionnaire } \\
\text { (quality of life items, score range } 0 \text {, best } \\
\text { case, to } 18 \text {, worst case); mean total } \\
\text { score Incontinence Impact Question- }\end{array}$ & $\begin{array}{l}\text { The mean quality } \\
\text { of life score ranged } \\
\text { across control } \\
\text { groups from } 39.08 \\
\text { to } 47\end{array}$ & $\begin{array}{l}\text { The mean quali- } \\
\text { ty of life score in } \\
\text { the intervention } \\
\text { groups was } \\
24.38 \text { to } 44\end{array}$ & $\begin{array}{l}\text { SMD }-0.91 \\
(-1.50 \text { to }-0.32)\end{array}$ & $\begin{array}{l}55 \text { participants } \\
(2)\end{array}$ & $\begin{array}{l}\oplus \oplus \odot \odot \\
\text { Low }^{b}\end{array}$ & - \\
\hline
\end{tabular}




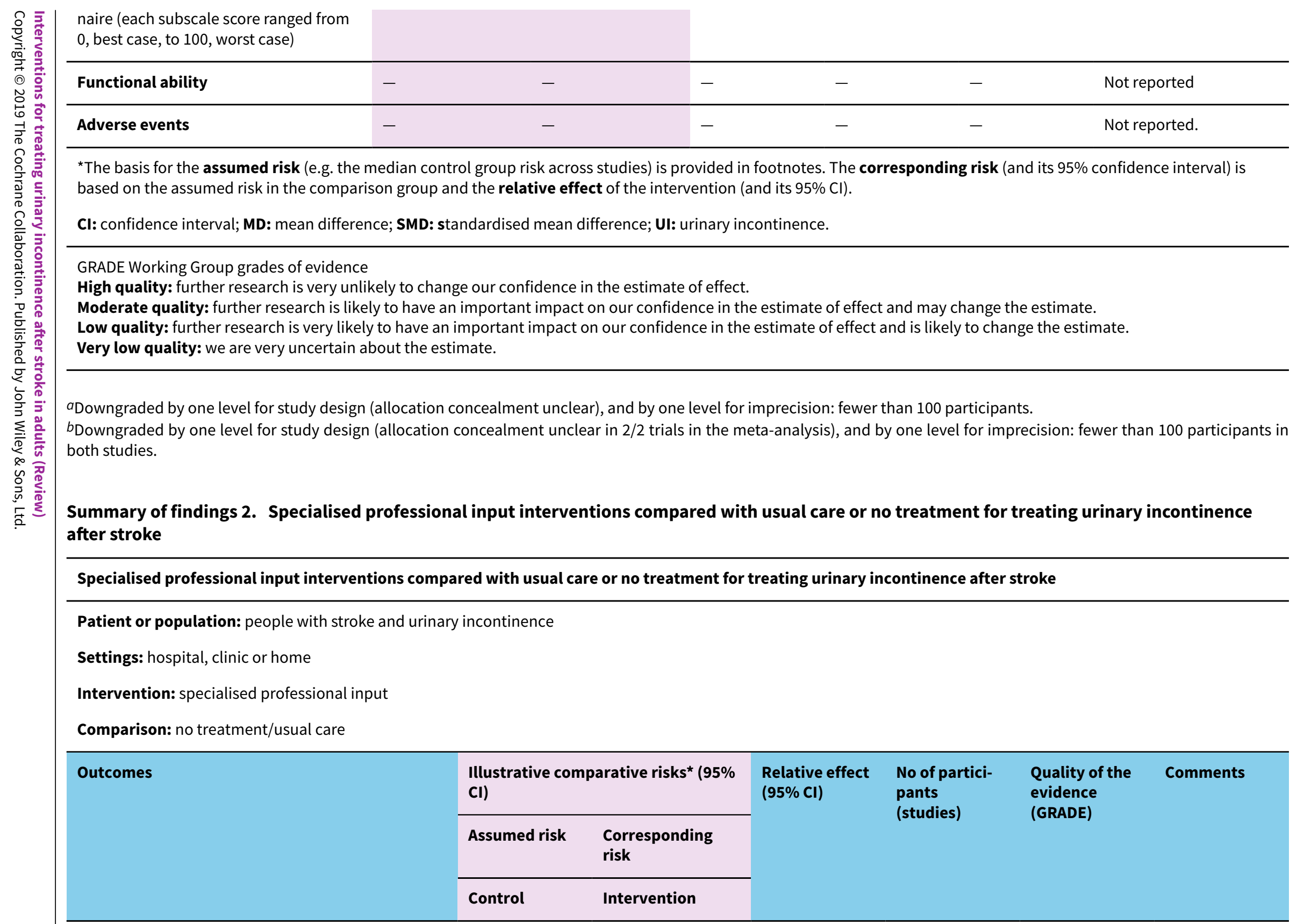




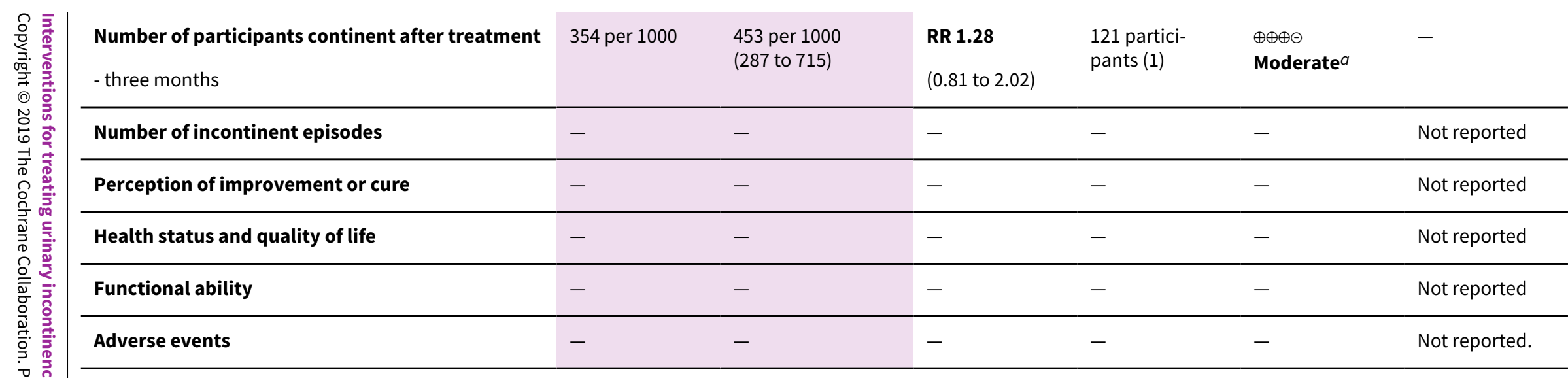

${ }^{*}$ The basis for the assumed risk (e.g. the median control group risk across studies) is provided in footnotes. The corresponding risk (and its $95 \%$ confidence interval) is based on the assumed risk in the comparison group and the relative effect of the intervention (and its $95 \% \mathrm{Cl}$ ).

Cl: confidence interval; RR: risk ratio.

GRADE Working Group grades of evidence

High quality: further research is very unlikely to change our confidence in the estimate of effect.

Moderate quality: further research is likely to have an important impact on our confidence in the estimate of effect and may change the estimate.

Low quality: further research is very likely to have an important impact on our confidence in the estimate of effect and is likely to change the estimate.

Very low quality: we are very uncertain about the estimate.

${ }^{a}$ Downgraded by one level for study design (allocation concealment unclear).

Summary of findings 3. Complementary therapy interventions compared with usual care or no treatment for treating urinary incontinence after stroke

Complementary therapy interventions compared with usual care or no treatment for treating urinary incontinence after stroke

Patient or population: people with stroke and urinary incontinence

Settings: hospital, clinic or home

Intervention: complementary therapy

Comparison: no treatment/usual care

\begin{tabular}{lllll}
\hline Outcomes & $\begin{array}{l}\text { Illustrative comparative risks } \\
(95 \% \mathrm{Cl})\end{array}$ & $\begin{array}{l}\text { Relative effect } \\
(95 \% \mathrm{Cl})\end{array}$ & $\begin{array}{l}\text { No of partici- } \\
\text { pants } \\
\text { (studies) }\end{array}$ & $\begin{array}{l}\text { Quality of the } \\
\text { evidence } \\
\text { (GRADE) }\end{array}$
\end{tabular}




\begin{tabular}{|c|c|c|c|c|c|c|}
\hline & $\begin{array}{l}\text { Assumed ris| } \\
\text { Control }\end{array}$ & $\begin{array}{l}\begin{array}{l}\text { Corresponding } \\
\text { risk }\end{array} \\
\text { Intervention }\end{array}$ & & & & \\
\hline \multirow{2}{*}{$\begin{array}{l}\text { Number of participants } \\
\text { continent after treat- } \\
\text { ment }\end{array}$} & \multicolumn{2}{|c|}{ Study population } & \multirow{2}{*}{$\begin{array}{l}\text { RR } 2.82 \\
\text { (1.57 to } 5.07)\end{array}$} & \multirow{2}{*}{$\begin{array}{l}524 \text { partici- } \\
\text { pants (5) }\end{array}$} & \multirow{2}{*}{$\begin{array}{l}\oplus \oplus \ominus \ominus \\
\text { Low } a\end{array}$} & \multirow[t]{2}{*}{-} \\
\hline & 193 per 1000 & $\begin{array}{l}544 \text { per } 1000 \\
(303 \text { to } 978)\end{array}$ & & & & \\
\hline $\begin{array}{l}\text { Number of incontinent } \\
\text { episodes }\end{array}$ & - & - & - & - & - & Not reported \\
\hline $\begin{array}{l}\text { Perception of improve- } \\
\text { ment or cure }\end{array}$ & - & - & - & - & - & Not reported \\
\hline $\begin{array}{l}\text { Health status and quality } \\
\text { of life }\end{array}$ & - & - & - & - & - & Not reported \\
\hline Functional ability & - & - & - & - & - & Not reported \\
\hline Adverse events & - & - & - & - & See comment & $\begin{array}{l}\text { Song 2013: } 45 / 136(33 \%) \text { in the intervention } \\
\text { group had bruises on arms and torso with full } \\
\text { recovery; } 17 / 136(13 \%) \text { had abdominal pain } \\
\text { post-acupuncture with resolution after warm } \\
\text { compress; no other adverse effects noted. } \\
\text { Chu 1997; Liu 2006; Zhang 2002; Zhou 1999: } \\
\text { unclear }\end{array}$ \\
\hline
\end{tabular}

${ }^{\star}$ The basis for the assumed risk (e.g. the median control group risk across studies) is provided in footnotes. The corresponding risk (and its $95 \%$ confidence interval) is based on the assumed risk in the comparison group and the relative effect of the intervention (and its $95 \% \mathrm{Cl}$ ).

Cl: confidence interval; RR: risk ratio.

GRADE Working Group grades of evidence

High quality: further research is very unlikely to change our confidence in the estimate of effect.

Moderate quality: further research is likely to have an important impact on our confidence in the estimate of effect and may change the estimate.

Low quality: further research is very likely to have an important impact on our confidence in the estimate of effect and is likely to change the estimate.

Very low quality: we are very uncertain about the estimate.

aDowngraded one level for study design (random sequence generation unclear in $3 / 5$ trials in the meta-analysis; allocation concealment unclear in $5 / 5$ trials), and by one level for imprecision: $3 / 5$ studies fewer than 100 participants. 
Physical therapy interventions compared with usual care or no treatment for treating urinary incontinence after stroke

Patient or population: people with stroke and urinary incontinence

Settings: hospital, clinic or home

Intervention: physical therapy

Comparison: no treatment/usual care

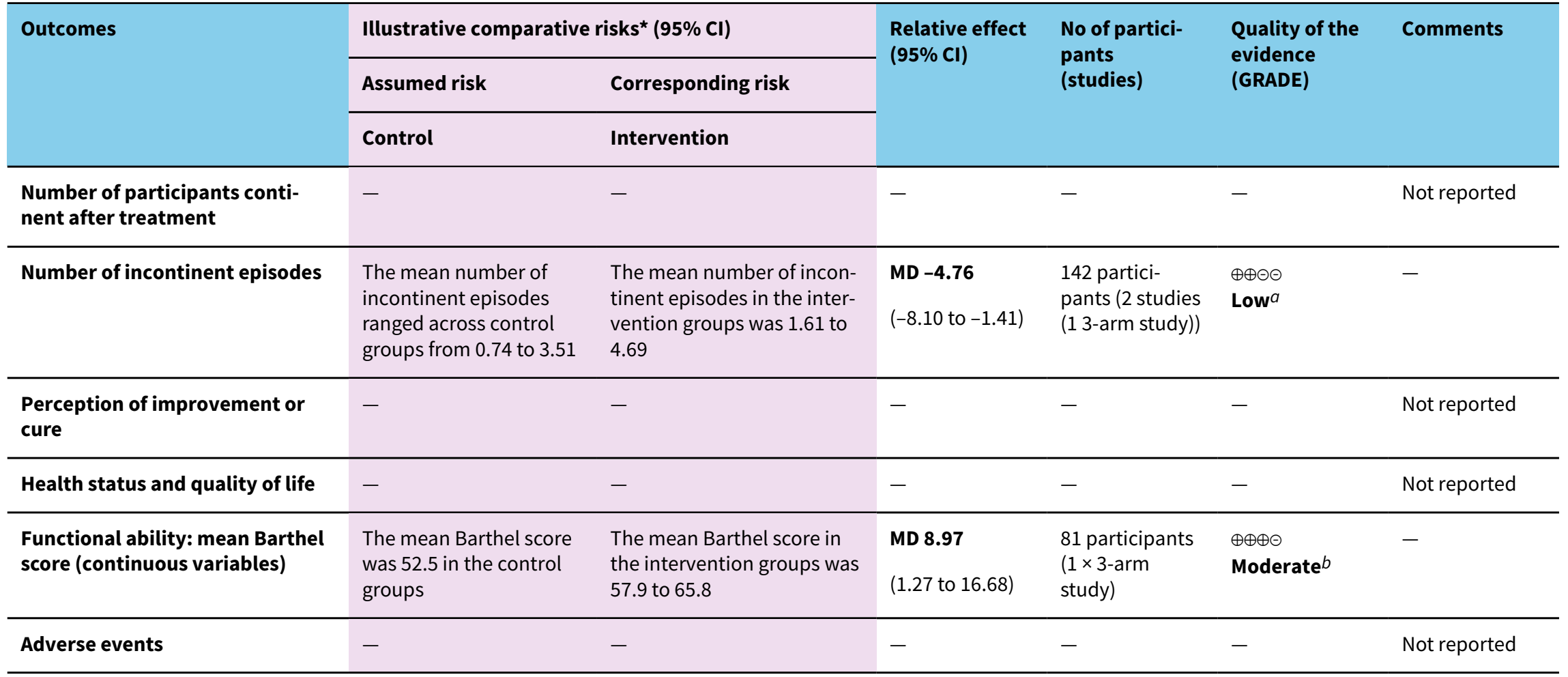

*The basis for the assumed risk (e.g. the median control group risk across studies) is provided in footnotes. The corresponding risk (and its $95 \%$ confidence interval) is based on the assumed risk in the comparison group and the relative effect of the intervention (and its $95 \% \mathrm{Cl}$ ).

Cl: confidence interval; MD: mean difference.

GRADE Working Group grades of evidence

High quality: further research is very unlikely to change our confidence in the estimate of effect.

Moderate quality: further research is likely to have an important impact on our confidence in the estimate of effect and may change the estimate. 


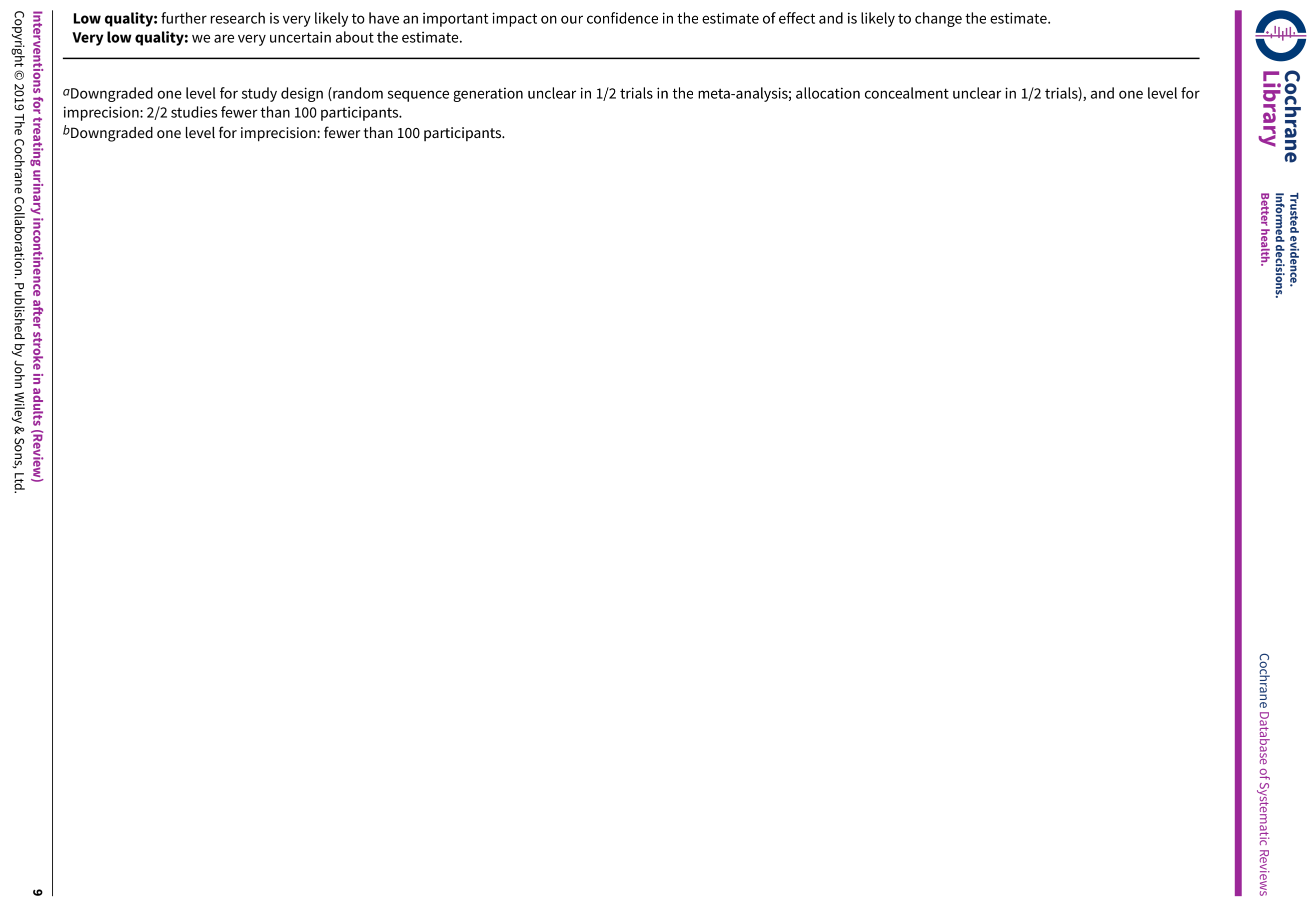




\section{B A C K G R O U N D}

Urinary incontinence (UI) may affect around half of stroke survivors in the acute phase of stroke recovery (during the first month). A significant proportion of stroke survivors, just less than half, will still be incontinent at three months and just over one-third of stroke survivors will remain incontinent at 12 months after stroke. This review focused on interventions for $\mathrm{UI}$ in stroke survivors who were at least one month post-stroke and considered to be in the rehabilitative phase of stroke recovery. A review is planned for interventions for stroke survivors with $\mathrm{UI}$ in the first month after stroke and considered to be in the acute phase of stroke recovery.

\section{Description of the condition}

$\mathrm{UI}$ is defined as the complaint of any involuntary leakage of urine (Abrams 2002). Up to 53\% of stroke survivors report UI at four weeks after stroke (Kolominsky-Rabas 2003). As many as $44 \%$ of stroke survivors are incontinent at three months post-stroke and $38 \%$ at one year after stroke (Williams 2012). UI often presents as a new problem after stroke or, if pre-existing, worsens significantly, adding to the disability and helplessness caused by neurological deficits (Brittain 2000a). The more severe the stroke, the greater the likelihood of UI (Brittain 1998b; Burney 1996a). Other factors include older age, female sex, speech difficulties, motor weakness, visual field defects or cognitive impairment (Barrett 2002).

Several studies have identified damage to the frontal lobe, the area believed to be responsible for control of micturition, as associated with urinary dysfunction after stroke. However, evidence suggests that the size of the lesion, rather than its location, is more likely to predict UI (Brittain 1999). It is unclear whether incontinence is a direct (i.e. site of brain lesion) or indirect (e.g. motor, visual or speech problems making the task of accessing toilet facilities a challenge) consequence of stroke. Other non-neurological factors contributing to UI, including premorbid continence state, sphincter incompetence and polyuria (Barrett 2002), are regarded as prevalent in the population of people with stroke (Brittain 1998b).

Problems experienced may range from urinary retention to complete incontinence. The most likely pattern of incontinence is urinary frequency, urgency (a sudden compelling desire to pass urine which is difficult to defer) and urge incontinence (involuntary leakage) (Marinkovic 2001). This is generally the result of detrusor overactivity (Talwar 1993), although this may depend on the site of the stroke lesion (Burney 1996b).

It is important to study $\mathrm{UI}$ in this population as symptoms are more severe and have more of an effect compared with other groups of people (Brittain 2000a). Incontinence is not just a physical problem but impacts on what people can do and how they feel (Williams 1993). Depression is twice as common in stroke survivors who are incontinent compared with those who are continent (Brittain 1998a; Limampai 2017). Negative social consequences for survivors and carers cannot be ignored; both may become isolated and marginalised (Brittain 2007).

Continuing incontinence is associated with poor outcome in both stroke survivors and carers (Arkan 2018; Nakayama 1997; Pettersen 2006; Tseng 2015). Conversely, stroke outcome is better in those stroke survivors who remain continent or regain continence (Barer 1989). Improvement is common over time (Marinkovic 2001), which suggests that problems with continence may be transient in some stroke survivors or amenable to intervention, or both. Factors predicting early improvements in continence status are less impairment on admission and the site of the stroke lesion (Ween 1996). Factors associated with poor recovery of continence include stroke type and being aged 75 or over (Patel 2001).

Incontinence is a strong predictor of stroke functional outcome (Meijer 2003). While there are problems with attributing better stroke outcome to improvements in continence, it is possible that recovery from incontinence may improve morale and self-esteem and, therefore, speed overall stroke recovery (Barer 1989; Patel 2001).

\section{Description of the intervention}

Current guidelines for the management of $\mathrm{UI}$ recommend an assessment to guide management (Intercollegiate Stroke Working Party 2016). This begins with physical assessment and historytaking, including identification of urological problems before the stroke occurred such as bladder outlet obstruction or stress incontinence. The choice of method to promote continence will then depend on the person's history and type of incontinence. Behavioural interventions are recommended as first-line therapy for managing UI (NICE 2012). These include interventions designed to promote continence, for example bladder training (appropriate for urge incontinence) and pelvic floor muscle training (appropriate for stress incontinence (Hay-Smith 2011)), and toileting assistance programmes such as prompted or timed voiding or habit retraining. These are designed to minimise incontinence episodes and are appropriate for people experiencing problems after stroke such as memory loss or restriction of movement (Eustice 2000; Ostaszkiewicz 2004a; Ostaszkiewicz 2004b; Roe 2007).

Other management techniques include: specialised professional input interventions (e.g. specialist continence advisors); complementary therapy interventions (e.g. acupuncture; Lim 2015); homeopathy; drug treatments (e.g. anticholinergics; Nabi 2006); physical therapies (e.g. electrical stimulation); physical aids (e.g. pessaries) and environmental or lifestyle interventions (e.g. diet and fluid management).

\section{How the intervention might work}

\section{Behavioural interventions}

Behavioural interventions are recommended as a first-line therapy for managing UI (NICE 2012). Bladder training aims to help people regain bladder control and continence through individualised voiding regimens designed to restore regular, normal voiding patterns by progressively lengthening the time interval between voids. PFMT involves contracting the pelvic floor muscles, raising the position of the levator ani muscles through increased volume and muscle stiffness, and, through this, providing more support to the bladder neck and proximal urethra (Ayeleke 2015). The duration, number and intensity of pelvic muscle floor contractions required to successfully treat $\mathrm{UI}$ is unclear. Timed voiding, prompted voiding and habit retraining (individualised toileting patterns) are examples of other behavioural interventions (International Continence Society 2015). 


\section{Specialised professional input interventions}

Specialised professional input interventions may include continence services provided by specialist nursing or continence teams in either a community or hospital setting. Professionals may include a range of staff, including general practitioners (GPs), continence nurse practitioners (CNPs), continence advisors, physiotherapists or occupational therapists. Individualised care pathways are implemented following holistic and in depth assessment, which may include urodynamic investigations. Interventions may include education or behavioural components.

\section{Complementary therapy interventions}

Complementary therapy interventions include acupuncture or homeopathy. Traditionally, acupuncture has been used in the treatment of pain relief (Paik 2013); this treatment method is now used for UI (Song 2011). In clinical practice, traditional manual acupuncture or electroacupuncture are the most commonly used techniques (Ju 2017), which involve the stimulation of specific acupuncture points on the skin by using thin disposable needles (VanderPloeg 2009). In treatment for the urinary system, acupuncture exerts an influence on both the autonomic and somatic nervous systems that control the muscles used in micturition and control UI (Paik 2013). The detrusor muscle is relaxed by sympathetic stimulation originating in the lumbar spinal cord region (T11-L2) and contracted by parasympathetic stimulation from the sacral spinal cord region (S2-4) (Paik 2013). The external urethral sphincter is under somatic control (Shefchyk 2001). Therapists report stimulating multiple acupoints in the treatment of UI (sacral, abdominal or leg regions of the body) but there is inconsistency in the depth of acupuncture (Paik 2013).

\section{Pharmacotherapy interventions}

Pharmacotherapy interventions may include anticholinergics (antimuscarinic agents), adrenergics or hormonal treatment. Anticholinergic drugs treat symptoms of overactive bladder, including incontinence, and act by decreasing the spontaneous muscle spasms of the bladder (Kuteesa 2006). Adrenergic agonists (alpha and beta) may be of benefit in promoting continence in the lower urinary tract by exerting effects on the contraction strength of the bladder neck and urethral sphincter muscle (alpha). Beta-adrenergics may promote continence through effects of the relaxation of the detrusor muscle, with or without contraction of the urethral sphincter (Alhasso 2005). Hormonal (oestrogen) therapy administered locally or systemically, may be prescribed for postmenopausal women and may help to keep the bladder and urethra healthy and functioning properly (Cody 2012). Oestrogen cream may help some women with UI by reversing atrophic vaginitis and urethritis. The effect of hormonal treatments in people post-stroke is unclear.

\section{Physical therapy interventions}

Physical therapy interventions include electrical stimulation or biofeedback. Neuromodulation of the sacral nerve plexus using electrical stimulation is an effective treatment to alleviate overactive bladder and urge UI (Staskin 2012). However, such an approach has not been previously tested in the stroke population. Electrically stimulating the sacral nerve plexus neuromodulates the L4-S3 nerve roots that control bladder function and urethral sphincter activity to eliminate inappropriate detrusor contractions while leaving the micturition reflex intact. Transcutaneous posterior tibial nerve stimulation (TPTNS) is a technique of noninvasive retrograde electrical stimulation of the sacral plexus via the sciatic nerve. The posterior tibial nerve, a tributary of the sciatic nerve, is accessed using surface electrodes applied to the medial malleolar area.

\section{Physical aids}

Physical aids are used to stop or control urinary leakage and include intravaginal devices (pessaries). Pessaries are used in stress $\mathrm{UI}$ to support the bladder neck. Some devices include a knob which compresses the urethra against the pubic bone to prevent leakage when intra-abdominal pressure increases (Al-Shaikh 2018).

\section{Environmental or lifestyle interventions}

Nutritional and metabolic mechanisms can affect the urinary tract. Lifestyle modifications (e.g. fluid and caffeine intake, smoking cessation, weight loss) may reduce UI. For example, weight loss may reduce higher intra-abdominal pressure associated with obesity through strengthening pelvic floor support structures (Imamura 2015).

\section{Why it is important to do this review}

This review aimed to evaluate the effects of a range of interventions designed to improve UI at least one month post-stroke by assessing the evidence available from randomised and quasi-randomised controlled trials. Current clinical guidelines are largely based on expert consensus (Intercollegiate Stroke Working Party 2016); there is little evidence of the effectiveness of interventions recommended (e.g. timed voiding, prompted voiding, bladder training) with the stroke population.

\section{OBJECTIVES}

To assess the effects of interventions for treating urinary incontinence after stroke in adults at least one month post-stroke.

\section{METHODS}

\section{Criteria for considering studies for this review}

\section{Types of studies}

We included randomised and quasi-randomised trials evaluating the effects of interventions designed to promote continence in people who have had a stroke. Quasi-random methods include allocation by the person's date of birth, by the day of the week or month of the year, by a person's medical record number or allocating every alternate person.

\section{Types of participants}

Adults (i.e. 18 years of age and over) with a diagnosis of stroke, including people with incontinence who have had a stroke identified as a subgroup within a larger group for whom relevant data were reported.

\section{Types of interventions}

One arm of the study must have included an intervention designed to promote urinary continence. We included trials evaluating any of the following in this review:

- behavioural interventions, for example prompted or scheduled voiding, bladder training, habit retraining (i.e. identification 
of voiding pattern and development of an individualised toileting schedule), PFMT or other behavioural management programmes;

- specialised professional input interventions, for example provision of information or education, assessment schedules, generic multidisciplinary rehabilitation programmes, continence advisors, home-support programmes or CNPs;

- complementary therapy interventions, for example homeopathy, acupuncture (traditional manual acupuncture or electroacupuncture);

- pharmacotherapy interventions, for example anticholinergics, adrenergics, hormonal treatment;

- physical therapy interventions, for example electrical stimulation, biofeedback;

- physical aids, for example pessaries, other appliances; and

- environmental or lifestyle interventions, for example voiding position, diet and fluid management.

Acceptable control interventions were usual care, no treatment, placebo or attention control (clinical attention in the form of an intervention inducing an expectation of therapeutic benefit; Freedland 2011). We believe that the comparison of particular interest to patients and practitioners in this review is intervention versus no intervention/usual care.

We excluded trials relating solely to surgical or physical interventions for pre-existing continence problems that were not associated with stroke (e.g. transurethral resection of the prostate) unless it was a cointervention in a wider trial testing an included method of continence promotion. We excluded trials relating to urological diagnosis, or to the management of incontinence or retention of urine in the acute phase of stroke (defined as up to one-month post-stroke). We also excluded trials if continence was not measured either by reporting participant symptoms or by a physical measure (e.g. a pad test).

\section{Types of outcome measures}

\section{Primary outcomes}

- Continence, measured by the following.

- Number of participants continent after treatment

- Number of incontinent episodes (indicated by bladder charts, total and mean number of episodes)

- Perception of improvement or cure (as reported by participant or carer)

\section{Secondary outcomes}

- Urinary symptoms, including frequency, urgency, nocturia

- Physical measures (e.g. pad tests of quantified leakage, postvoid retention of urine, void volume, urodynamic measures)

- Health status and quality of life (impact of incontinence e.g. Incontinence Impact Questionnaire (IIQ), 36-Item Short Form Health Survey Questionnaire (SF-36), Bristol Female Urinary Symptoms Questionnaire, knowledge, quality of life)

- Functional ability (activities of daily living e.g. Barthel Index)

- Participant satisfaction

- Adverse events

\section{'Summary of findings' tables}

We used GRADE to interpret findings and to create 'Summary of findings' tables for the main comparison (intervention versus no intervention/usual care) using the outcomes below for behavioural interventions, specialised professional input, complementary therapy and physical therapy:

- number of participants continent after treatment;

- number of incontinent episodes in 24 hours;

- perception of improvement or cure;

- health status and quality of life;

- functional ability; and

- adverse events.

We chose outcomes for the 'Summary of findings' tables based on the primary outcomes, outcomes of clinical importance, and outcomes of most importance to patients.

\section{Search methods for identification of studies}

We imposed no language or other restrictions on any of the searches.

\section{Electronic searches}

We used the search strategies developed for both Cochrane Incontinence and Cochrane Stroke. We initially identified relevant trials from the Groups' Specialised Registers of controlled trials.

For more details of the search methods used to build the Cochrane Incontinence Specialised Register, see the Group's webpages where details of the Register's development (from inception) and the most recent searches performed to populate the Register can be found. For more details of the search methods used to build the Cochrane Stroke Specialised Register, see the Cochrane Stroke webpages.

To summarise, both Registers contain trials identified from the Cochrane Central Register of Controlled Trials (CENTRAL), MEDLINE, MEDLINE In-Process, MEDLINE Epub Ahead of Print, CINAHL, ClinicalTrials.gov, WHO ICTRP, UK Clinical Research Network Portfolio, and handsearching of journals and conference proceedings. The Cochrane Stroke Specialised Register includes searches of many other sources of trials. Many of the trials in the Cochrane Incontinence and Cochrane Stroke Specialised Registers are also contained in CENTRAL. The dates of the most recent searches of the Specialised Registers for this review update were: 30 October 2017 (Cochrane Stroke Register) and 1 November 2017 (Cochrane Incontinence Register).

The terms used to search the Cochrane Incontinence and Cochrane Stroke Specialised Registers are given in Appendix 1.

For the first two versions of this review, extra specific searches were performed (Thomas 2005; Thomas 2008). For more details, including the search terms used, see Appendix 2.

\section{Searching other resources}

We searched the reference lists of all relevant reviews and trial reports to identify further relevant studies. 


\section{Data collection and analysis}

\section{Selection of studies}

Two review authors (of LT, JC and LC) independently screened titles and abstracts for potentially eligible studies. We resolved any disagreements regarding the inclusion or exclusion of individual studies by discussion or, if necessary, by consulting the third review author. We contacted authors of identified articles where there were missing or unclear data in order to inform study selection decisions. There was no masking of the source and authorship of the trial reports.

\section{Data extraction and management}

Pairs of review authors (of LT, JC and LC) performed independent data extraction of the included trials using a piloted data collection form. The data collected included information on study design, study population, interventions, outcomes measurement and results. We resolved any discrepancies in data extraction either by discussion between the two authors or with involvement of the third review author. Two review authors (LT and JC) entered data into Review Manager 5 (Review Manager 2014).

\section{Assessment of risk of bias in included studies}

Two review authors (LT and JC) independently assessed the risk of bias of included studies using Cochrane's 'Risk of bias' tool (Higgins 2011). The tool covers the domains of random sequence generation, allocation concealment, blinding of participants and personnel, blinding of outcome assessment, incomplete outcome data, selective outcome reporting and other bias. We classified each domain as either low risk, high risk or unclear risk of bias.

\section{Measures of treatment effect}

We based analyses on available data from all included trials relevant to the comparisons and outcomes of interest. We summarised effect estimates for continuous outcomes using mean difference (MD) and we summarised dichotomous outcomes using risk ratio (RR). For continuous outcome data, we used MD of posttreatment scores unless changes from baseline data were available.

\section{Unit of analysis issues}

For cross-over trials, we analysed the data using a paired samples mean and SE test. We analysed multi-arm trials comparing two interventions arms with one control group using methods described by Higgins 2011 (section 16.5.4). To prevent inappropriate double-counting of individuals, we analysed each treatment arm separately against the common control group but divided the sample size of the common comparator group proportionately across each intervention comparison.

\section{Dealing with missing data}

We attempted to obtain missing data, as well as data collected but not reported, by contacting trialists. We contacted study authors of trials which included a subgroup of people with stroke to obtain stroke subgroup data. If no response was received from study authors after one contact, we made a second request to obtain the required data.

\section{Assessment of heterogeneity}

We only combined and pooled data from trials if the types of interventions were similar enough to do so. We investigated differences between trials if heterogeneity $\left(\mathrm{I}^{2}\right)$ was greater than $50 \%$. If heterogeneity could not be explained, we considered using a random-effects model.

We described the statistical heterogeneity of the intervention effects by calculating the $\mathrm{I}^{2}$ statistic and using the $\mathrm{Chi}^{2}$ test. We interpreted heterogeneity as follows.

- $0 \%$ to $40 \%$ : represents low heterogeneity.

- $30 \%$ to $60 \%$ : may represent moderate heterogeneity.

- $50 \%$ to $90 \%$ : may represent substantial heterogeneity.

- $75 \%$ to $100 \%$ : represents considerable heterogeneity.

We used both random-effects and fixed-effect meta-analysis with $95 \% \mathrm{Cl}$ using Review Manager (Review Manager 2014). We pooled outcomes such as quality of life measured with different instruments using the standardised mean difference (SMD).

\section{Assessment of reporting biases}

We searched clinical trial registers to assist in reducing publication bias. We also investigated selective outcome reporting though the comparison of the methods section of papers with the results reported.

\section{Data synthesis}

Dichotomous outcomes reported as favourable events were the number of people continent after treatment and participant satisfaction. Outcomes reported as unfavourable events were number of incontinence episodes in 24 hours, urinary symptoms (including frequency, urgency, nocturia) and adverse events.

We summarised effect estimates for continuous outcomes using MD or SMD and we summarised dichotomous outcomes using RR. Where deemed appropriate, we pooled effects across similar studies using fixed- or random-effects meta-analysis techniques with $95 \%$ confidence intervals (Cls). We used random-effects metaanalysis (DerSimonian 1986) if the studies showed heterogeneity (defined by the studies' effects having an $\mathrm{I}^{2}$ statistic of greater than $50 \%$ ); otherwise, we used a fixed-effect analysis (Mantel-Haenszel method) for dichotomous and inverse variance for continuous data (Mantel 1959). For continuous outcome data, we used change from baseline data if available; otherwise, we used the raw outcome data.

\section{Subgroup analysis and investigation of heterogeneity}

Where data were available, we planned subgroup analyses for the effect of urological diagnosis (i.e. detrusor overactivity versus other) and time from stroke onset to recruitment to trial.

\section{Sensitivity analysis}

Where data allowed, we planned to explore the effects of including studies assessed as having a high risk of bias using sensitivity analyses.

\section{'Summary of findings' tables}

We prepared 'Summary of findings' tables for the main comparison. To ensure clarity, we created individual 'Summary of findings' tables for specific clinically important interventions within the main comparison. 
Three review authors (LT, JC, LC) assessed and documented the quality of evidence for the prespecified outcomes outlined in the Types of outcome measures based on the GRADE approach (Guyatt 2008). We downgraded the evidence from high-quality by one level for serious (or by two levels for very serious) study limitations:

- risk of bias due to flawed design or conduct of studies (random sequence generation, allocation concealment, blinding of participants and personnel, blinding of outcome assessment, and incomplete outcome data). We reassessed all studies from the original review (Thomas 2008) using the updated 'Risk of bias' tool (Higgins 2011);

- imprecision (e.g. when Cls for treatment effect were wide);

- inconsistency (e.g. when point estimates varied widely, the $\mathrm{I}^{2}$ was large);

- indirectness (e.g. variations in participants, interventions, comparisons and outcomes); and

- publication bias (may be explored with the use of funnel plots and classed as not suspected, suspected, strongly suspected or very strongly suspected).

\section{RES U LTS}

\section{Description of studies}

\section{Results of the search}

\section{Search for the 2019 review update}

The previous version of this review included 12 studies (Thomas 2008). The search for the 2019 version identified a further 10 studies (Booth 2016; Chu 2011; Guo 2014; Liu 2013; Liu 2016a; Liu 2016b; Monteiro 2014; Shin 2016a; Song 2013; Tibaek 2017; Zhang 1996; see Figure 1). One review author (AT) translated studies published in Chinese. Two studies were reported in the same conference abstract (Gelber 1997a; Gelber 1997b). Two trials originally included in the 2008 version were removed from this update as they were found not to be eligible (Wikander 1998; Zhu 2003). Two studies previously classified as awaiting assessment were excluded in this version as stroke subgroup data were not available (Engberg 2002; McDowell 1999). We identified two potentially relevant studies that are still ongoing (NCT02568774; Shin 2016b). We classified three studies as awaiting further assessment (ACTRN12617000162314; ChiCTR-INR-16010239; Wang 2014). 
Figure 1. PRISMA study flow diagram (2019 review update).

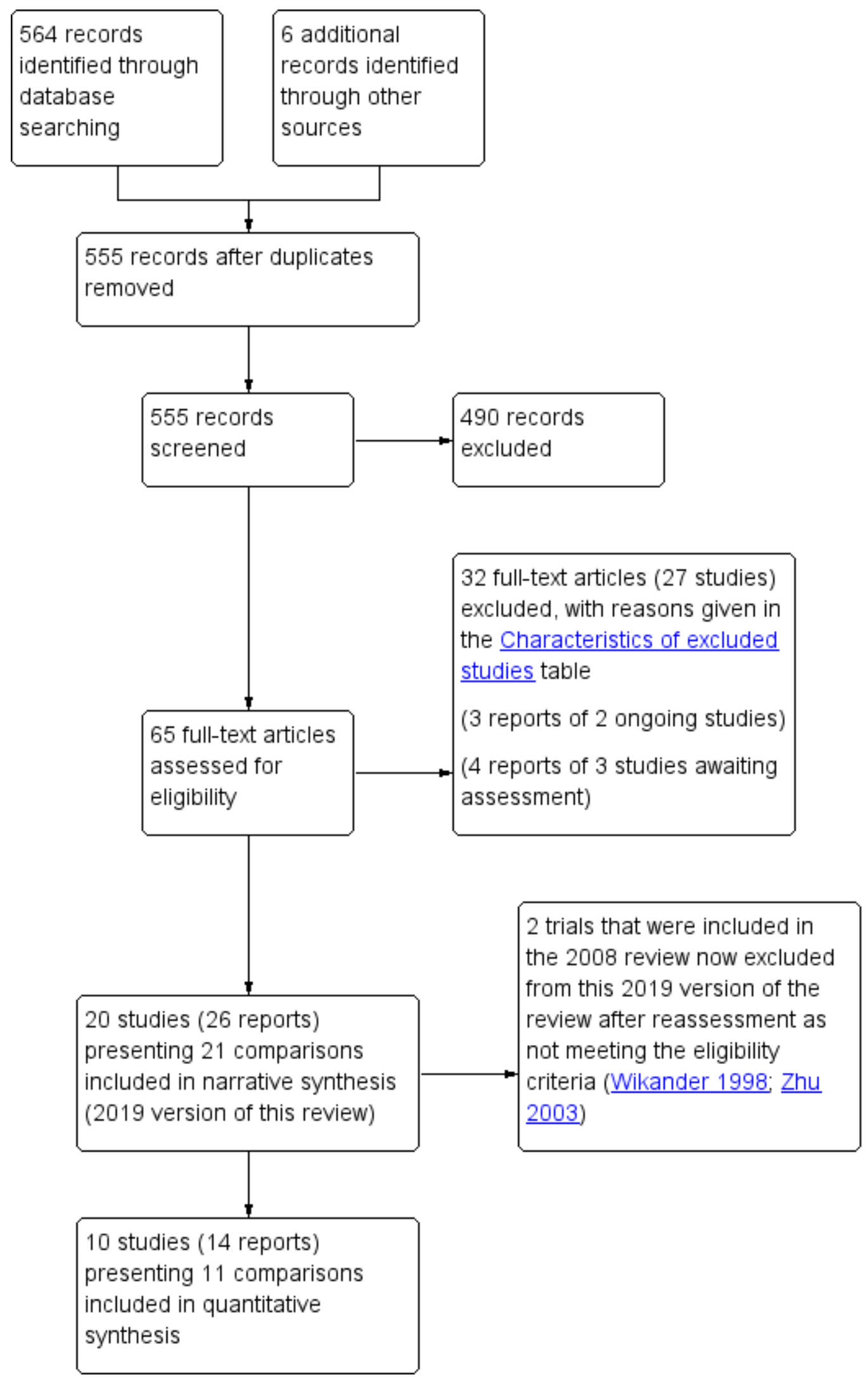


Therefore, the review has 20 included studies (reporting 21 comparisons), 27 excluded studies, two ongoing studies and three studies awaiting classification.

Appendix 3 shows the results of the search for the previous versions of this review (Thomas 2005; Thomas 2008).

\section{Included studies}

For details about the included studies, please see the Characteristics of included studies. Please note: Liu 2016a and Liu 2016b are two arms of a multi-arm RCT reporting on two separate comparisons. Henceforth, they are listed below as "Liu 2016a and Liu 2016b" to highlight their relation to one another.

\section{Design}

Of the 20 included trials, all were randomised controlled trials with the exception of Booth 2016, which was a randomised controlled feasibility trial and Judge 1969, which was a cross-over trial. The trial by Liu was a three-arm trial (Liu 2016a and Liu 2016b). All trials were single-centre with the exception of Booth 2016 and Song 2013.

\section{Study funding sources}

Twelve studies did not publish a funding statement (Chu 1997; Chu 2011; Gelber 1997a; Gelber 1997b; Guo 2014; Lewis 1990; Liu 2006; Monteiro 2014; Song 2013; Zhang 1996; Zhang 2002; Zhou 1999). Two studies reporting three comparisons reported that they received no funding grants (Liu 2016a and Liu 2016b; Shin 2016a). Six studies reported funding sources (Booth 2016; Brittain 2000b; Judge 1969; Liu 2013; Tibaek 2005; Tibaek 2017).

\section{Study dates}

Publication dates of the trials ranged from 1969 to 2016. Six studies did not report information on the period when participants were recruited to the trials or the dates the studies were conducted (Gelber 1997a; Gelber 1997b; Judge 1969; Lewis 1990; Shin 2016a; Zhang 1996). Five trials reported the study period as occurring between 1992 and 2001 (Brittain 2000b; Chu 1997; Tibaek 2005; Zhang 2002; Zhou 1999). One trial was conducted between 2004 and 2006 (Liu 2006). Four trials were conducted between 2007 and 2012 (Liu 2013; Monteiro 2014; Song 2013; Tibaek 2017). One trial was conducted between 2014 and 2016 (Booth 2016). One trial reported recruitment from hospital inpatients during the period 2007 to 2010 (Chu 2011). One trial reported hospitalisation dates of participants between 2010 and 2011 (Guo 2014). Liu 2016a and Liu 2016b reported that participants were in hospital between 2011 and 2013.

\section{Sample sizes}

Three trials included fewer than 25 participants (Judge 1969; Lewis 1990; Monteiro 2014). Five trials included between 26 and 49 participants (Gelber 1997a; Gelber 1997b; Shin 2016a; Tibaek 2005; Tibaek 2017). Eleven trials, including one trial that reported two comparisons, had 50 or more participants (Booth 2016; Brittain 2000b; Chu 1997; Chu 2011; Guo 2014; Liu 2006; Liu 2013; Liu 2016a and Liu 2016b; Zhang 1996; Zhang 2002; Zhou 1999).

\section{Setting}

Of the 20 trials, 10 were carried out in China, including one trial that reported two comparisons (Chu 1997; Chu 2011; Guo 2014; Liu 2006; Liu 2013; Liu 2016a and Liu 2016b; Song 2013; Zhang 1996; Zhang
2002; Zhou 1999), one in England (Brittain 2000b), two in Scotland (Booth 2016; Judge 1969), three in the USA (Gelber 1997a; Gelber 1997b; Lewis 1990), one in Brazil (Monteiro 2014), one in Korea (Shin 2016a), and two in Denmark (Tibaek 2005; Tibaek 2017).

Six trials took place in an outpatient setting (Guo 2014; Liu 2013; Monteiro 2014; Shin 2016a; Tibaek 2005; Tibaek 2017). One trial took place in a long-stay inpatient setting for elderly people (Judge 1969). Two trials took place in an inpatient setting (Liu 2006; Zhang 1996), and two trials, including one trial that reported two comparisons, were conducted in both an inpatient and outpatient setting (Liu 2016a and Liu 2016b; Song 2013). Two trials took place in participants' homes in the community (Booth 2016; Brittain $2000 \mathrm{~b})$. The remaining trials did not specify the location of care (Chu 1997; Chu 2011; Gelber 1997a; Gelber 1997b; Lewis 1990; Zhang 2002; Zhou 1999).

\section{Participants}

The 20 trials included 1338 participants. The numbers of participants in the individual trials ranged from 12 to 232 . Twelve trials, including one trial reporting two comparisons, included both genders (Booth 2016; Brittain 2000b; Chu 1997; Chu 2011; Guo 2014; Liu 2006; Liu 2013; Liu 2016a and Liu 2016b; Song 2013; Zhang 1996; Zhang 2002; Zhou 1999). Five trials included only women (Judge 1969; Shin 2016a; Tibaek 2005), or only men (Monteiro 2014; Tibaek 2017). Three trials did not report gender characteristics (Gelber 1997a; Gelber 1997b; Lewis 1990).

Three trials did not report the age of the participants (Gelber 1997a; Gelber 1997b; Lewis 1990). Six trials reported an age range of participants (Brittain 2000b: 40 to 96 years; Chu 1997: 45 to 71 years; Judge 1969: 66 to 92 years; Zhang 1996: 52 to 78 years; Zhang 2002: 42 to 62 years; Zhou 1999: 52 to 85 years). Eleven trials reported the mean and standard deviation (SD) of the ages of the participants in both the intervention and control groups. Of these, eight had a mean age in the intervention group of 62 to 68 years, including one trial that reported two different comparisons (Booth 2016; Chu 2011; Guo 2014; Liu 2006; Liu 2016a and Liu 2016b; Monteiro 2014; Shin 2016a; Tibaek 2017). Two trials reported a lower mean age in the intervention group: Liu 2013 (mean age 39 years) and Song 2013 (mean age 55 years). One trial reported a median age of 60 years (interquartile range (IQR) 56 to 74 years) (Tibaek 2005).

Eleven trials reported both inclusion and exclusion criteria, including one trial that reported two different comparisons (Booth 2016; Brittain 2000b; Guo 2014; Liu 2006; Liu 2013; Liu 2016a and Liu 2016b; Monteiro 2014; Shin 2016a; Song 2013; Tibaek 2005; Tibaek 2017). Seven trials did not report any inclusion and exclusion criteria (Chu 1997; Gelber 1997a; Gelber 1997b; Lewis 1990; Zhang 1996; Zhang 2002; Zhou 1999). One trial reported exclusion criteria only (Judge 1969), while one trial reported inclusion criteria only (Chu 2011).

Six trials, including one trial reporting two comparisons, included participants with either a first or recurrent stroke (i.e. only including participants after a first stroke was not mentioned in either inclusion or exclusion criteria) (Booth 2016; Guo 2014; Liu 2016a and Liu 2016b; Monteiro 2014; Shin 2016a; Tibaek 2017). With the exception of Tibaek 2005, it was not possible to determine whether only participants with a first stroke were included in the remaining trials. 


\section{Interventions}

The trials tested the following interventions.

Behavioural interventions (five trials):

- timed voiding versus void on request (Gelber 1997a);

- timed voiding versus oxybutynin (Gelber 1997b);

- PFMT versus usual care (Shin 2016a; Tibaek 2005; Tibaek 2017).

Specialised professional input interventions (one trial):

- care from a CNP versus usual care provided by the GP (Brittain 2000b).

Complementary therapy interventions (eight trials):

- scalp acupuncture versus no scalp acupuncture (Chu 1997);

- eye acupuncture and scalp electroacupuncture versus herbal medication therapy (Zhou 1999);

- traditional acupuncture (knee and inside of ankle) versus usual care (Zhang 2002);

- pelvic plexus acupuncture versus filiform needle (Zhang 1996);

- ginger-salt-partitioned moxibustion (involving filling the navel with salt, adding a piece of ginger and a taper and setting the taper alight) plus routine acupuncture versus routine acupuncture (Liu 2006);

- electroacupuncture (lumbar and sacral region) versus standard medical therapy and scalp and body acupuncture (Chu 2011);

- electroacupuncture combined with traditional acupuncture (sacral region) versus sham acupuncture (sacral region) (Liu 2013);

- electroacupuncture (multiple points on arms, legs and torso) versus indwelling catheter with bladder training (Song 2013).

Pharmacotherapy interventions (one trial):

- oestrogen versus placebo (Judge 1969).

Physical therapy interventions (five trials reporting six comparisons):

- sensory-motor biofeedback device (Uristop) combined with timed voiding against timed voiding alone (Lewis 1990);

- transcutaneous posterior tibial nerve stimulation (TPTNS) versus sham (Booth 2016), or versus attention control (Monteiro 2014);

- transcutaneous electrical nerve stimulation (TENS) versus usual care (Guo 2014), no treatment control or a different frequency of stimulation (Liu 2016a and Liu 2016b).

We found no trials evaluating interventions that included physical aids, or environmental or lifestyle interventions.

\section{Intervention comparisons}

The effects of the included intervention types (behavioural, specialised professional input, complementary, pharmacotherapy and physical therapy) were considered within the following five comparison categories:

- intervention versus no intervention/usual care;

- intervention versus placebo;
- specific intervention versus another intervention;

- combined intervention versus single intervention;

- specific intervention versus attention control.

\section{Intervention versus no intervention/usual care}

Twelve trials tested an intervention versus no intervention or usual care, including one trial reporting two comparisons (Brittain 2000b; Chu 1997; Gelber 1997a; Guo 2014; Liu 2006; Liu 2016a and Liu 2016b; Shin 2016a; Song 2013; Tibaek 2005; Tibaek 2017; Zhang 2002; Zhou 1999).

\section{Behavioural interventions}

One trial tested a behavioural intervention comprising timed voiding versus void on request for participants with normal urodynamic studies (Gelber 1997a). Normal urodynamic studies were not defined and no further details were given of the intervention. Two trials tested an intensive PFMT programme, comprising individual and group exercises and feedback to participants, compared with normal rehabilitation with no specific treatment of UI (Tibaek 2005; Tibaek 2017). One trial evaluated PFMT in addition to general rehabilitation training compared with general rehabilitation training alone (Shin 2016a).

\section{Specialised professional input interventions}

One trial tested a specialised professional input intervention comparing care given by a CNP versus usual care provided by a GP and existing specialised services for the management of continence (Brittain 2000b).

\section{Complementary therapy interventions}

Five trials tested complementary interventions: Chu 1997 tested scalp acupuncture plus usual care versus usual care; Zhang 2002 tested acupuncture versus general treatment; and Zhou 1999 tested eye and scalp electroacupuncture versus herbal medication therapy only, which we classed as usual care. Liu 2006 tested gingersalt-partitioned moxibustion plus routine acupuncture versus routine acupuncture. Song 2013 compared electroacupuncture versus indwelling catheter.

\section{Physical therapy interventions}

Two trials tested transcutaneous electrical nerve stimulation. In one trial, the comparison group received "basic therapy" (Guo 2014). The other trial, reporting two comparisons, compared different electrical frequencies against no treatment (Liu 2016a and Liu 2016b).

\section{Intervention versus placebo}

One cross-over trial tested a pharmacotherapy intervention (oestrogen) versus placebo (Judge 1969).

One feasibility trial compared TPTNS versus a sham intervention with electrodes positioned to avoid the tibial nerve (Booth 2016). One trial compared electroacupuncture trial combined with traditional acupuncture with sham acupuncture (Liu 2013).

\section{Specific intervention versus another intervention}

Gelber 1997b tested a specific intervention against another intervention, comparing the anticholinergic oxybutynin versus timed voiding in participants with bladder hyper-reflexia. Zhang 
1996 tested acupuncture with an elongated needle compared with a filiform needle.

\section{Combined intervention versus single intervention}

One trial tested a combined intervention (sensory-motor biofeedback device combined with timed voiding) versus a single intervention (timed voiding alone) (Lewis 1990).

\section{Specific intervention versus attention control}

Monteiro 2014 compared 12 sessions of electrical stimulation of the posterior tibialis nerve versus an attention control group. Participants in the attention control group undertook a task involving 12 sessions of muscle-stretching training exercises of the lower limb.

\section{Diagnosis}

\section{Pre-stroke continence status}

Only 11 trials, reporting 12 comparisons, included participants who were continent prior to the stroke (Booth 2016; Chu 2011; Guo 2014; Liu 2006; Liu 2013; Liu 2016a and Liu 2016b; Monteiro 2014; Shin 2016a; Song 2013; Tibaek 2005; Tibaek 2017). Nine trials did not specify whether UI was subsequent to the occurrence of stroke (Brittain 2000b; Chu 1997; Gelber 1997a; Gelber 1997b; Judge 1969; Lewis 1990; Zhang 1996; Zhang 2002; Zhou 1999), although Lewis 1990 described participants as having "post-stroke urinary urge incontinence."

Further information on the continence status of participants prior to stroke as defined by study authors can be found in Table 1 .

\section{Diagnostic criteria for urinary incontinence used by study authors}

Eight trials reporting nine comparisons gave diagnostic criteria for incontinence (Brittain 2000b; Chu 2011; Guo 2014; Liu 2013; Liu 2016a and Liu 2016b; Shin 2016a; Tibaek 2005; Tibaek 2017). Four trials specified a urological diagnosis: normal urodynamic studies (Gelber 1997a); bladder hyper-reflexia (Gelber 1997b); urge incontinence (Lewis 1990); and urge, stress and mixed stress/urge incontinence (Tibaek 2005). Judge 1969 reported data for two groups of participants defined as mildly or severely incontinent. Booth 2016 reported data on post-stroke participants who reported incontinence at least once per week. Liu 2006 reported data for three groups of participants, classified according to the Barthel continence item: completely incontinent, partially incontinent and independent. Liu 2006 and Monteiro 2014 reported data using the Barthel Index (bladder item). Chu 1997 included participants who had "urinary frequency or urinary incontinence" but did not define urinary frequency further. One trial reported participants met the "urological criteria for incontinence" with no further details available in the translation (Song 2013). Three trials did not include a urological diagnosis for participants or group them by type or severity of incontinence (Zhang 1996; Zhang 2002; Zhou 1999).

For further information on the diagnostic criteria for incontinence and the description of incontinence by study authors, see Table 1.

\section{Diagnostic criteria for stroke used by study authors}

Booth 2016, Tibaek 2005 and Tibaek 2017 diagnosed stroke according to the World Health Organization's clinical definition, which includes confirmation by computed tomography (CT) or magnetic resonance imaging (MRI) scan (WHO 1989). Similarly, in Liu 2013 and Zhang 1996, a physician diagnosed stroke and observed cerebral haemorrhage or infarction on cranial CT or MRI. Tibaek 2005 included only first-ever ischaemic strokes. All participants in the intervention arm of Tibaek 2017 had no previous history of stroke. Song 2013 diagnosed stroke according to 1995 National Meeting on Cerebrovascular Accidents diagnostic criteria. Chu 2011 included participants diagnosed with first or repeated episode of stroke according to the Fourth National Conference on Cerebrovascular Disease. Monteiro 2014 assessed participants with clinical and neuroimaging evaluations according to established criteria from the Brazilian Cerebrovascular Disease Society. Eleven trials reporting 12 comparisons did not report stroke diagnostic criteria (Chu 1997; Gelber 1997a; Gelber 1997b; Guo 2014; Judge 1969; Lewis 1990; Liu 2006; Liu 2016a and Liu 2016b; Shin 2016a; Zhang 2002; Zhou 1999).

Participants in 10 trials reporting 11 comparisons included those with cerebral infarction and haemorrhage (Booth 2016; Chu 2011; Guo 2014; Lewis 1990; Liu 2006; Liu 2013; Liu 2016a and Liu 2016b; Song 2013; Zhang 1996; Zhou 1999). Two trials included only participants with infarction (Tibaek 2005; Zhang 2002). In Zhang 2002, almost half of the participants had multiple cerebral infarctions. Judge 1969 included participants with cerebrovascular accident or "multiple little strokes." Two trials reported that strokes were unilateral (Gelber 1997a; Gelber 1997b), while one trial reported the stroke type as multi-focal infarction (Chu 1997). Brittain 2000b included participants who self-reported that they had had a stroke, subarachnoid haemorrhage or transient ischaemic attack on a screening questionnaire.

Participants in Liu 2006 were 70.74 (SD 35.26) days poststroke. Three trials reporting four comparisons similarly recruited participants between seven and 10 weeks post-stroke (Guo 2014; Liu 2016a and Liu 2016b; Tibaek 2017). Zhou 1999 presented findings for participants who were less than or more than three months post-stroke. Shin 2016a reported participants were more than three months post-stroke. Participants in three trials were also less likely to be in the early rehabilitation phase, as they were either occupying long-stay geriatric hospital beds or living at home (Brittain 2000b; Judge 1969; Tibaek 2005). Booth 2016 recruited people between zero and more than five years after stroke. Of these, $12.2 \%$ (8 participants) were between zero and three months, and $30.6 \%$ (15 participants) were more than five years post-stroke. It was difficult to identify the phase of stroke recovery for participants in the other trials (Chu 1997; Gelber 1997a; Gelber 1997b; Lewis 1990; Liu 2013; Monteiro 2014; Zhang 1996; Zhang 2002).

Please see Table 2 for further information on the participants' stroke history, stroke diagnostic information and stroke description.

\section{Description of outcomes}

Fifteen of the 20 trials clearly stated the primary outcome(s) of interest in the trial. In six trials, this was the number of people with UI (Brittain 2000b; Chu 1997; Chu 2011; Zhang 1996; Zhang 2002; Zhou 1999); in nine trials it was number of incontinent episodes (Booth 2016; Gelber 1997a; Gelber 1997b; Judge 1969; Lewis 1990; Liu 2006; Liu 2013; Tibaek 2005; Tibaek 2017). Four trials had an additional primary outcome of urinary symptoms (Liu 2006; Liu 2013; Tibaek 2005; Tibaek 2017). Five trials reporting six comparisons did not report a primary outcome (Guo 2014; Liu 2016a and Liu 2016b; Monteiro 2014; Shin 2016a; Song 2013). 
Please see Table 3 for further information on primary and secondary outcomes as described by study authors.

\section{Excluded studies}

We excluded 27 studies. Reasons for exclusion can be found in the Characteristics of excluded studies but are summarised below.

- Four studies did not include a measure of continence (Cook 1998; Gross 1990; Humphreys 2014; Moon 2012).

- Five studies recruited participants in the acute phase of stroke (Gong 2013; Thomas 2011; Wikander 1998; Yun 2007; Zhu 2003); in addition to recruitment in the acute phase, Yun 2007 did not include a measure of continence.

- Six studies had stroke participants as a subgroup within their overall participants but data for these participants were not available or reported (Engberg 2002; Kuo 2007; McDowell 1999; NCT00213577; Sakakibara 2008; Stohrer 2013).

- Six studies did not include participants with stroke (EUCTR2009-009216-53-PT; Gousse 2007; Kim 2003; Madersbacher 2004; Madersbacher 2005; Vinsnes 2010).

- Three studies were terminated due to poor recruitment and reported no data (Byles 2006; NCT01275261; Shin 2016c).
- We were unable to trace the author for further publications or information for two studies (ISRCTN97151578; Smilskalne 2009).

- One study did not directly test a method of promoting continence (Tekeoglu 1998).

\section{Studies awaiting classification}

We classified three studies as awaiting further assessment (ACTRN12617000162314; ChiCTR-INR-16010239; Wang 2014). Please see the Characteristics of studies awaiting classification for details.

\section{Ongoing studies}

We identified two potentially eligible studies that are still ongoing (NCT02568774; Shin 2016b). For more information, please see the Characteristics of ongoing studies.

\section{Risk of bias in included studies}

The risk of bias judgements are summarised in Figure 2 and Figure 3 , and described in the 'Risk of bias' tables in the Characteristics of included studies.

\section{Figure 2. Risk of bias graph: review authors' judgements about each risk of bias item presented as percentages across all included studies.}

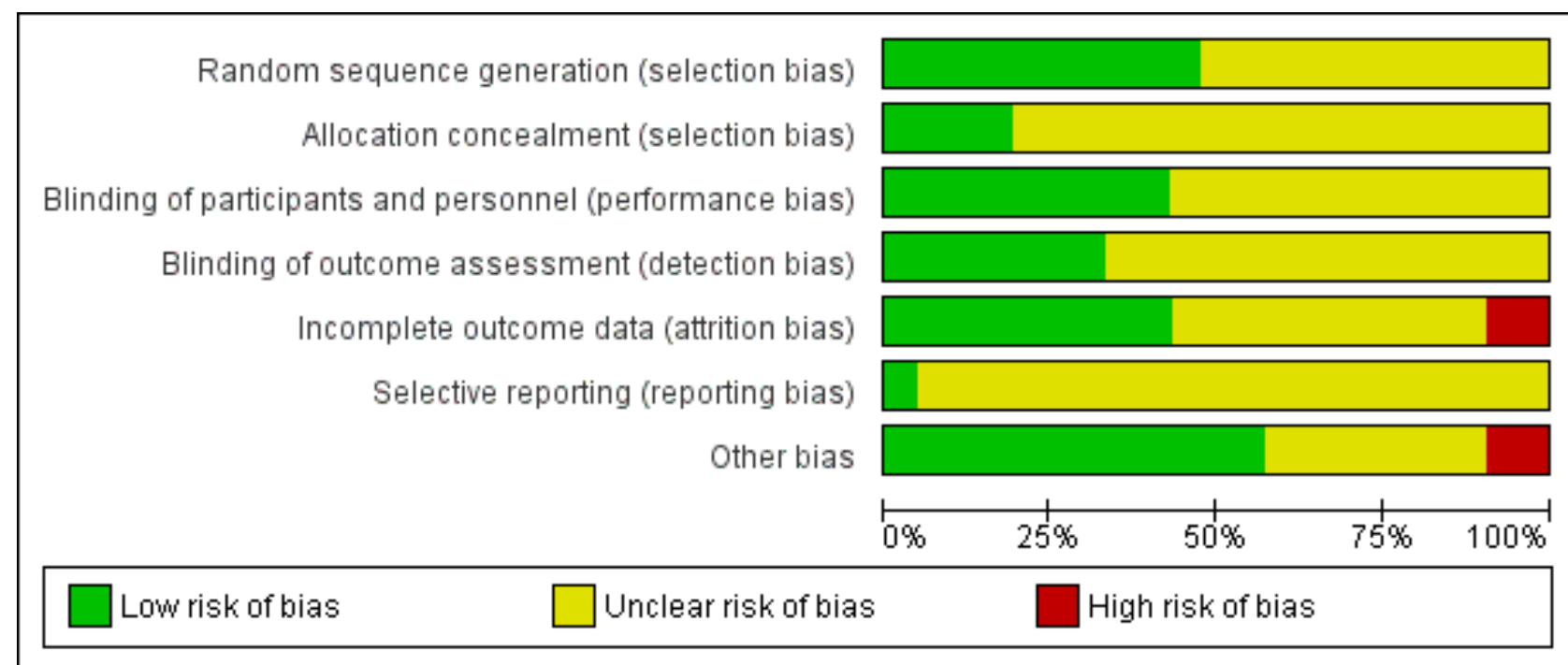


Figure 3. Risk of bias summary: review authors' judgements about each risk of bias item for each included study.

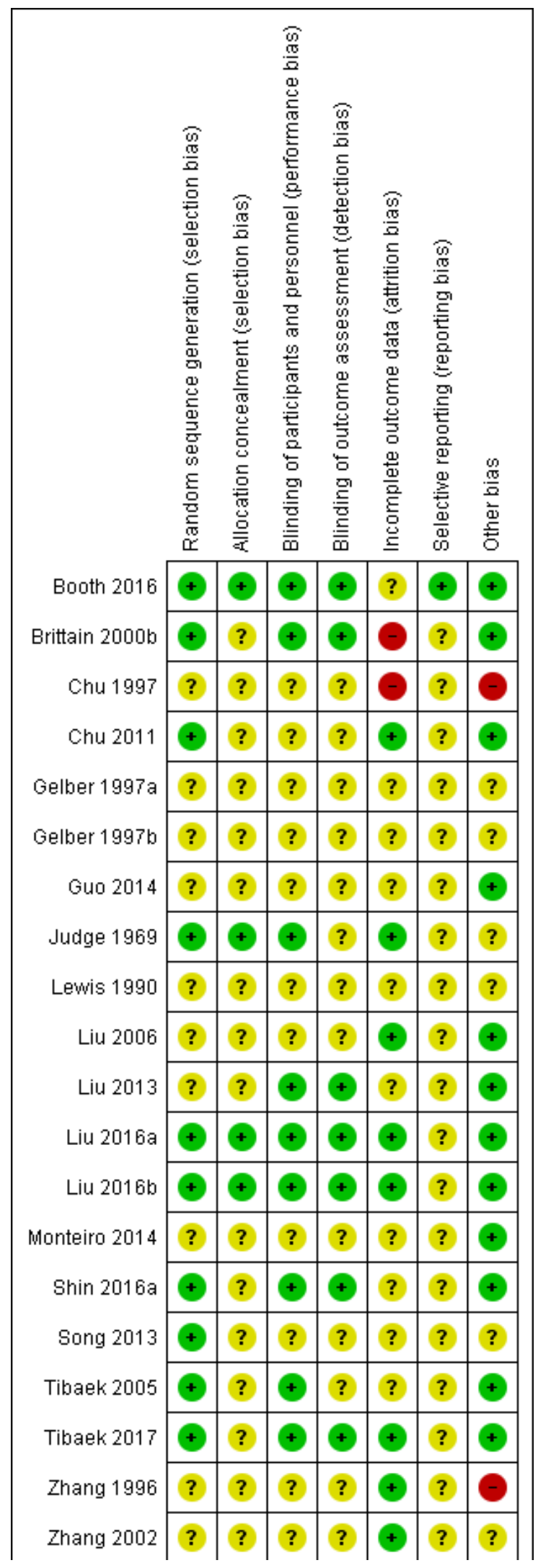


Figure 3. (Continued)

\begin{tabular}{|c|c|c|c|c|c|c|c|}
\cline { 2 - 6 } Zhang 2002 & $?$ & $?$ & $?$ & $?$ & + & $?$ & $?$ \\
\cline { 2 - 7 } Zhou 1999 & $?$ & $?$ & $?$ & $?$ & + & $?$ & $?$ \\
\hline
\end{tabular}

\section{Allocation}

\section{Random sequence generation}

Nine trials reporting 10 comparisons were at low risk of bias for random sequence generation (Booth 2016; Brittain 2000b; Chu 2011; Judge 1969; Liu 2016a and Liu 2016b; Shin 2016a; Song 2013; Tibaek 2005; Tibaek 2017). The remaining trials were at unclear risk of bias.

\section{Allocation (selection bias)}

Three trials reporting four comparisons were at low risk of bias for allocation concealment (Booth 2016; Judge 1969; Liu 2016a and Liu 2016b). The remaining trials were at unclear risk of bias.

\section{Blinding}

\section{Blinding of participants and personnel (performance bias)}

Eight trials reporting nine comparisons were at low risk of bias for blinding of participants or personnel, or both (Booth 2016; Brittain 2000b; Judge 1969; Liu 2013; Liu 2016a and Liu 2016b; Shin 2016a; Tibaek 2005; Tibaek 2017). The remaining trials were at unclear risk of bias.

\section{Blinding of outcome assessment (detection bias)}

Six trials reporting seven comparisons were at low risk of bias for blinding of outcome assessors (Booth 2016; Brittain 2000b; Liu 2013; Liu 2016a and Liu 2016b; Shin 2016a; Tibaek 2017). The remaining trials were at unclear risk of bias.

\section{Incomplete outcome data}

Eight trials reporting nine comparisons were at low risk of bias in relation to incomplete outcome data (Chu 2011; Judge 1969; Liu 2006; Liu 2016a and Liu 2016b; Tibaek 2017; Zhang 1996; Zhang 2002; Zhou 1999). Two trials were at high risk of bias due to the large amount of attrition (Brittain 2000b), and lack of prespecification of outcomes (Chu 1997). The remaining trials were at unclear risk of bias.

\section{Selective reporting}

One study was at low risk of reporting bias as it had a published protocol (Booth 2016). The remaining studies were at unclear risk of bias as there were no study protocols available.

\section{Other potential sources of bias}

Eleven trials reporting 12 comparisons were free from other sources of bias (Booth 2016; Brittain 2000b; Chu 2011; Guo 2014; Liu 2006; Liu 2013; Liu 2016a and Liu 2016b; Monteiro 2014; Shin 2016a; Tibaek 2005; Tibaek 2017). Two trials were at high risk of other bias due to insufficient description of care received by the comparison group and no assessment of participant characteristics at baseline respectively (Chu 1997; Zhang 1996). The remaining trials were at unclear risk of bias.
None of the trials reported power calculations, with the exception of Tibaek 2017. Booth 2016 explicitly did not use a power calculation as this was a feasibility trial.

\section{Effects of interventions}

See: Summary of findings for the main comparison Behavioural interventions compared with usual care or no treatment for treating urinary incontinence after stroke; Summary of findings 2 Specialised professional input interventions compared with usual care or no treatment for treating urinary incontinence after stroke; Summary of findings 3 Complementary therapy interventions compared with usual care or no treatment for treating urinary incontinence after stroke; Summary of findings 4 Physical therapy interventions compared with usual care or no treatment for treating urinary incontinence after stroke

For information relating to the quality of evidence for behavioural, specialised professional input, complementary therapy and physical therapy interventions for the main comparison (intervention versus no intervention/usual care), please refer to Summary of findings for the main comparison, Summary of findings 2, Summary of findings 3 and Summary of findings 4 .

Data for prespecified outcomes were not available for each comparison except where reported below.

\section{Intervention versus no intervention/usual care (comparison 1)}

Twelve trials reporting 13 comparisons with 986 participants compared an intervention to promote urinary continence against no intervention or usual care (Brittain 2000b; Chu 1997; Gelber 1997a; Guo 2014; Liu 2006; Liu 2016a and Liu 2016b; Shin 2016a; Song 2013; Tibaek 2005; Tibaek 2017; Zhang 2002; Zhou 1999). The interventions included:

- behavioural interventions

- timed voiding versus void on request (usual care) (Gelber 1997a)

- PFMT versus usual care (Shin 2016a; Tibaek 2005; Tibaek 2017)

- specialised professional input interventions

- care from a CNP versus usual care provided by a GP (Brittain 2000b)

- complementary therapy interventions

- scalp acupuncture versus no scalp acupuncture (Chu 1997)

- eye acupuncture and scalp electroacupuncture versus no acupuncture (Zhou 1999)

- knee and ankle acupuncture versus usual care (Zhang 2002)

- ginger-salt-partitioned moxibustion plus routine acupuncture (multiple points) versus routine acupuncture (Liu 2006)

- electroacupuncture (multiple points) versus indwelling catheter (Song 2013)

- physical therapy interventions 
- TENS versus basic therapy (Guo 2014)

- TENS (two groups with different frequencies) versus no treatment (Liu 2016a and Liu 2016b)

No data were available for the other interventions.

\section{Primary outcomes}

\section{Number of participants continent after treatment}

Six trials (including 766 people) reported the number of participants who were continent after treatment (Brittain 2000b; Chu 2011; Liu 2006; Song 2013; Zhang 2002; Zhou 1999). Randomeffects models were used for the meta-analysis because of heterogeneity. The six trials included one specialised professional input intervention (Brittain 2000b); and five complementary therapy interventions (Chu 2011; Liu 2006; Song 2013; Zhang 2002; Zhou 1999).

\section{Specialised professional input interventions}

Based on one trial of moderate-quality, specialist professional input interventions in the form of structured assessment and management probably made little or no difference to the number of people continent at three months after treatment (RR 1.28, 95\% $\mathrm{Cl} 0.81$ to 2.02; Brittain 2000b; Analysis 1.1.1; Summary of findings 2). At six months, the CNP intervention may have made little or no difference to the number of people continent after treatment (16/91 $(17.5 \%)$ in the treatment group versus $8 / 55(14.6 \%)$ in the control group; RR $0.96,95 \% \mathrm{Cl} 0.83$ to 1.11 ; analysis not shown; Brittain 2000b).

\section{Complementary therapy interventions}

Acupuncture and ginger-salt-partitioned moxibustion may have increased the number of participants continent after treatment (RR 2.82, $95 \% \mathrm{Cl} 1.57$ to 5.07 ; 524 participants; low-quality evidence; Chu 2011; Liu 2006; Song 2013; Zhang 2002; Zhou 1999; Analysis 1.1.2; Summary of findings 3). Studies were heterogeneous $\left(I^{2}=\right.$ $78 \%$ ) but favoured complementary therapy using the randomeffects model. Visual inspection of the forest plot suggested there was a larger effect size in Chu 2011 and Zhang 2002. It is difficult to suggest a possible explanation for this difference in treatment effect. However, Chu 2011 was the only trial to use electroacupuncture rather than acupuncture and Zhang 2002 included younger participants, ranging from 42 to 62 years.

\section{Number of incontinent episodes}

\section{Behavioural interventions}

One trial measured the mean number of incontinent episodes in 24 hours (Tibaek 2005). Behavioural interventions may have made little or no difference to the number of incontinent episodes (MD $-1.00,95 \% \mathrm{Cl}-2.74$ to $0.74 ; 26$ participants; $\mathrm{P}=0.26$; lowquality evidence; Analysis 1.2.1; Summary of findings for the main comparison). Tibaek 2017 reported the number of incontinent episodes in 24 hours post-intervention was very small, with no episodes in either the intervention or control group.

One trial compared timed voiding versus void on request, which was interpreted as usual care (Gelber 1997a). The data reported were too few even for tentative conclusions and we obtained no further data from the investigators. This study was reported as ongoing in a conference abstract.

\section{Physical therapy interventions}

TENS may have reduced the mean number of incontinent episodes in 24 hours (MD $-4.76,95 \% \mathrm{Cl}-8.10$ to $-1.41 ; 142$ participants; lowquality evidence; Guo 2014; Liu 2016a and Liu 2016b; Analysis 1.2.2; Summary of findings 4). There was a high level of heterogeneity $\left(I^{2}=\right.$ $94 \%$ ) but findings favoured TENS using the random-effects model. Visual inspection of the forest plot suggested there was a larger effect size in Liu 2016a and Liu 2016b. A possible explanation for this difference in treatment effect may come from the number of days patients received TENS (Guo 2014: mean number of days 48.7 (SD 10.5); Liu 2016a: mean number of days 65.96 (SD 9.39); Liu 2016b: mean number of days 71.01 (SD 14.86)).

\section{Perception of improvement or cure}

None of the trials reported perception of improvement or cure.

\section{Secondary outcomes}

Urinary symptoms - overall urinary symptoms, mean number of symptoms and leakage scores

\section{Specialised professional input interventions}

In Brittain 2000b, the CNP intervention may have slightly improved the proportion cured of all four urinary symptoms (frequency, nocturia, urgency and urinary incontinence) at three months. The number of participants cured of all four urinary symptoms was $24.7 \%$ in the treatment group versus $17.9 \%$ in the control group (147 participants).

At six months, Brittain 2000b found a larger proportion of people were cured of all four urinary symptoms in the treatment group $(41 / 89 ; 46.1 \%)$ compared with the control group $(16 / 54 ; 29.6 \%)$ (RR $1.55,95 \% \mathrm{Cl} 0.97$ to 2.48 , Analysis 1.3 .1$)$.

There were no data suitable for analysis in relation to changes in daytime and night-time leakage scores. However, the study teams reported that the CNP intervention possibly improved the daytime severity of leakage at three months $(P=0.038)$.

There were no data suitable for analysis in relation to changes in the mean number of symptoms. However, the CNP intervention may have slightly reduced the total number of symptoms experienced at three months $(P<0.01)$. The intervention may have made little or no difference to the total number of overall symptoms at six months $(P=0.06$; Brittain 2000b).

\section{Urinary symptoms - frequency}

\section{Behavioural interventions}

Data from two trials (48 participants) suggests that PFMT may have slightly improved mean daytime voiding frequency (MD -1.71, 95\% Cl-3.02 to -0.40; 48 participants; Tibaek 2005; Tibaek 2017; Analysis 1.4.1). There was no heterogeneity $\left(I^{2}=0 \%\right)$.

Tibaek 2017 followed up participants for six months. We are uncertain whether PFMT reduced voiding frequency: mean daytime frequency was 6.8 (SD 3.2) in the treatment group and 8 (SD 3) in the control group; mean night-time frequency was 2.1 (SD 1.7) in the treatment group and 2.2 (SD 1.6) in the control group (19 participants). 


\section{Specialised professional input interventions}

In Brittain 2000b, there was no evidence that the CNP intervention made any difference to urinary frequency at three months $(98 / 120$ $(82 \%)$ in the treatment group versus $59 / 67(88 \%)$ in the control group; RR $0.93,95 \% \mathrm{Cl} 0.82$ to 1.05 ; 187 participants; Analysis 1.5.1).

At six months, the CNP intervention may have made little or no difference to urinary frequency $(73 / 89(82 \%)$ in the treatment group versus $47 / 54(87 \%)$ in the control group; RR $0.94,95 \% \mathrm{Cl} 0.82$ to 1.09).

\section{Complementary therapy interventions}

One small trial suggested ginger-salt-partitioned moxibustion plus routine acupuncture may have reduced mean daytime voiding frequency (Liu 2006). The mean frequency of episodes was 7.03 in the intervention group compared with 12.6 in the control group (MD $-5.57,95 \% \mathrm{Cl}-7.00$ to $-4.14 ; 62$ participants; Analysis 1.4.2).

There were no data suitable for analysis in the trial of scalp acupuncture versus no scalp acupuncture (Chu 1997). After two weeks and two courses of treatment, the investigators reported a reduction in urinary frequency or incontinence of $90.3 \%$ in the intervention group, with two people not regaining "normal urine," 12 people partly regaining "normal urine" and 16 people regaining "normal urine". The authors reported a significant difference between the experimental and control groups as "p $\left(0.05^{\sim} 0.001\right) . "$ There were no reported results for the control group. The study authors provided no further data.

\section{Physical therapy interventions}

Based on two trials, one reporting two comparisons, TENS may have made no difference to daytime frequency (MD -2.83, 95\% Cl -5.75 to 0.09; 142 participants; Guo 2014; Liu 2016a and Liu 2016b; Analysis 1.4.3). The trials were heterogeneous $\left(I^{2}=93 \%\right)$. Visual inspection of the forest plot suggested there was a larger effect size in Liu 2016a and Liu 2016b. As we describe above in relation to Analysis 1.2.2, a possible explanation for this difference in treatment effect may come from the number of days patients received TENS (Guo 2014: mean number of days 48.7 (SD 10.5) days; Liu 2016a: mean number of days 65.96 (SD 9.39) days; Liu 2016b: mean number of days 71.01 (SD 14.86)).

\section{Urinary symptoms - urgency}

\section{Specialised professional input interventions}

In Brittain 2000b, there was no evidence that the CNP intervention made a difference to urgency at three months. The number of people reporting urgency was $95 / 121(79 \%)$ in the treatment group compared with 50/67 (75\%) in the control group (RR 1.05, 95\% Cl 0.89 to 1.24 ; 188 participants; Analysis 1.6.1).

At six months, the CNP intervention may have made little or no difference to urinary urgency $(65 / 91(71.4 \%)$ in the treatment group versus $40 / 54(74 \%)$ in the control group; RR $0.96,95 \% \mathrm{Cl} 0.79$ to 1.18).

\section{Urinary symptoms - nocturia}

\section{Behavioural interventions}

Two trials suggested PFMT may have made little or no difference in reducing nocturia (MD $-0.38,95 \% \mathrm{Cl}-1.06$ to $0.29 ; 48$ participants; $\mathrm{P}=0.26, \mathrm{I}^{2}=25 \%$; Tibaek 2005; Tibaek 2017; Analysis 1.7.1). This analysis used a random-effects model as it was not readily possible to switch a subgroup analysis to a fixed-effect model.

\section{Specialised professional input interventions}

One trial suggested the CNP intervention may have made little or no difference to nocturia (Brittain 2000b). The number of people reporting nocturia at three months was $102 / 119(86 \%)$ in the treatment group versus $60 / 67$ (90\%) in the control group (RR 0.96, $95 \% \mathrm{Cl} 0.86$ to 1.07 ; Analysis 1.8 .1 ).

At six months, the CNP intervention may also have made little or no difference to nocturia $(77 / 89(87 \%)$ in the treatment group versus $46 / 53$ (87\%) in the control group; RR $1.00,95 \% \mathrm{Cl} 0.87$ to 1.14 ).

\section{Complementary therapy interventions}

Two trials looking at ginger-salt-partitioned moxibustion and electroacupuncture respectively suggested that complementary therapies may have made little or no difference in reducing nocturia (MD -1.76, 95\% Cl -4.49 to 0.96; 256 participants; $\mathrm{P}=0.20$; Liu 2006; Song 2013; Analysis 1.7.2). The trials were heterogeneous $\left(I^{2}=98 \%\right)$. Visual inspection of the forest plot suggested there was a larger effect size in Liu 2006. A possible explanation for this difference in treatment effect could relate to the sample size: Liu 2006 had 62 participants included in the analysis, whereas Song 2013 had 194. Furthermore, the control group in Song 2013 had an indwelling catheter inserted: this is usually only justified if there are specific clinical reasons for insertion (for example when fluid balance is critical) given the high risk of urinary tract infection (ISWP 2016).

\section{Physical therapy interventions}

One trial suggested TENS may have improved nocturia (MD -1.52 , $95 \% \mathrm{Cl}-1.73$ to -1.31 ; 61 participants; Analysis 1.7.3; Guo 2014).

\section{Physical measures}

\section{Behavioural interventions}

One trial suggested PFMT may have made little or no difference to the mean function of the pelvic floor muscle (MD $0.10,95 \% \mathrm{Cl}-0.48$ to 0.68 ; 23 participants; Tibaek 2005; Analysis 1.9.1).

One trial reported median pelvic floor muscle strength postintervention of 5 (IQR 4 to 6) in the treatment group versus 4 (IQR 4 to 5) in the control group; 30 participants; $\mathrm{P}=0.07$; Tibaek 2017).

One trial followed up participants for six months. We were uncertain whether PFMT improved pelvic floor muscle strength (median 5 (IQR 4 to 6) in the treatment group versus 4 (IQR 3 to 5 ) in the control group; 24 participants; $\mathrm{P}=0.15$; Tibaek 2017 .

\section{Health status and quality of life}

\section{Behavioural interventions}

There was no evidence from one trial that PFMT made a difference to the mean total score of the SF-36 (MD $-28.00,95 \% \mathrm{Cl}-169.66$ to 113.66; 24 participants; Tibaek 2005; Analysis 1.10).

One trial measured quality of life using the mean total score on the IIQ (Tibaek 2005), and one trial used the Bristol Female Lower Urinary Tract Symptoms Questionnaire (quality of life items) (Shin 2016a). Data from these two trials provided no evidence of a difference to quality of life (SMD $-0.99,95 \% \mathrm{Cl}-2.83$ to 0.86 ; 55 participants; low-quality evidence; Shin 2016a; Tibaek 2005; Analysis 1.11.1; Summary of findings for the main comparison). 
The trials were heterogeneous $\left(I^{2}=90 \%\right)$. Visual inspection of the forest plot suggested there was a larger effect size in Shin 2016a . It is difficult to suggest a possible explanation for this difference in treatment effect, however Shin 2016a included participants around six months post-stroke whereas Tibaek 2005 included participants around 12 months post-stroke.

One trial measured health status using the SF-36 pre- and postintervention (Tibaek 2017). There were no significant differences reported and data were unsuitable for pooling.

One trial followed up participants for six months (Tibaek 2005). We were uncertain whether PFMT improved health status. Total scores on the SF-36 were 550 (SD 170) in the treatment group (12 participants) and 596 (SD 124) in the control group (12 participants) $(P=0.6$; Tibaek 2005). Higher scores on the SF-36 indicate a more favourable health status. There was no evidence that PFMT changed quality of life at six months: scores on the IIQ were 43 (SD 76) in the treatment group (12 participants) and 47 (SD 50) in the control group (11 participants) $(P=0.45)$.

One trial followed up participants for six months (Tibaek 2017). We were uncertain whether PFMT improved health status measured by the SF-36 (30 participants).

\section{Functional ability}

\section{Physical therapy interventions}

Two comparators of one three-arm trial (81 participants) measured function using the Barthel Index (Liu 2016a and Liu 2016b). TENS (Liu 2016a: $20 \mathrm{~Hz}$; Liu 2016b: 75 Hz frequencies) probably improved overall functional ability (MD $8.97,95 \% \mathrm{Cl} 1.27$ to 16.68 ; moderatequality evidence; Analysis 1.12).

\section{Participant satisfaction}

\section{Specialised professional input interventions}

One trial measured participant satisfaction with the service (Brittain 2000b). The CNP intervention may have improved participant satisfaction at three months (dissatisfaction: 13/109 $(12 \%)$ in the treatment group versus $17 / 45(38 \%)$ in the control group; RR 0.32, 95\% Cl 0.17 to 0.59; Analysis 1.13).

\section{Adverse events}

In Song 2013, 45/136 (33\%) of participants in the intervention group reported bruising on arms and torso with full recovery; 17/136 $(13 \%)$ had abdominal pain postacupuncture with resolution after warm compress. There were no other adverse effects.

Three trials reporting four comparisons reported no adverse events (Guo 2014; Liu 2016a and Liu 2016b; Tibaek 2017). Four trials reported no information on adverse events (Brittain 2000b; Gelber 1997a; Shin 2016a; Tibaek 2005). In four trials, it was unclear if any adverse events occurred (Chu 1997; Liu 2006; Zhang 2002; Zhou 1999).

\section{Intervention versus placebo (comparison 2)}

Three trials with 137 participants compared an intervention to promote urinary continence against placebo (Booth 2016; Judge 1969; Liu 2013). One reported on a physical therapy intervention (Booth 2016), one reported on pharmacotherapy in the form of oestrogen (Judge 1969), and the other reported on complementary therapy (Liu 2013).

\section{Primary outcomes}

Number of participants continent after treatment

\section{Physical therapy interventions}

Data from one trial provided no evidence that transcutaneous posterior nerve stimulation made a difference to the number of participants continent at 12 weeks (Booth 2016). The trial reported that $3 / 27$ (11.1\%) participants in the treatment group were continent compared with $4 / 27(14.8 \%)$ in the sham intervention group (RR 0.75, 95\% $\mathrm{Cl} 0.19$ to 3.04; Analysis 2.1).

\section{Number of incontinent episodes}

\section{Physical therapy interventions}

Data from one trial provided no evidence that TPTNS made a difference to the number of incontinence episodes (MD -1.10, 95\% $\mathrm{Cl}-3.99$ to 1.79; 39 participants; Booth 2016; Analysis 2.2).

One trial followed up participants for six months (Booth 2016). It is uncertain whether TPTNS reduced the number of UI episodes at follow-up (odds ratio (OR) $1.43,95 \% \mathrm{Cl} 0.38$ to 5.32 ).

\section{Pharmacotherapy interventions}

One small cross-over trial compared an intervention designed to promote urinary continence (oestrogen) against placebo in a longterm care setting in 12 women with a history of stroke (Judge 1969). The trial reported results separately for participants with mild or severe incontinence and in view of the significant heterogeneity between the two groups, we used a random-effects model.

There was no evidence that oestrogen therapy made a difference to the mean number of incontinent episodes per week in mild incontinence (paired samples for mild incontinence, MD -1.71, 95\% $\mathrm{Cl}-3.51$ to 0.09; Analysis 2.3.1) or severe incontinence (paired samples, MD -6.40, $95 \% \mathrm{Cl}-9.47$ to -3.33 ; Analysis 2.3.2).

\section{Perception of improvement or cure}

\section{Physical therapy interventions}

One trial reported that a single Patient Perception of Bladder Condition question suggested improvement in the TPTNS group at the 26-week time point (OR $0.04,95 \% \mathrm{Cl} 0.004$ to 0.41 ; Booth 2016). There was no evidence of a difference in perceived bladder condition at six weeks (OR 2.33, 95\% $\mathrm{Cl} 0.63$ to 8.65 ) and 12 weeks (OR $1.22,95 \% \mathrm{Cl} 0.29$ to 5.17 ).

\section{Secondary outcomes}

\section{Urinary symptoms}

\section{Complementary therapy interventions}

One trial (71 participants) found that acupuncture may have reduced the frequency of urination and UI (Liu 2013). The frequency of urination and $\mathrm{UI}$ were lower after electroacupuncture than before treatment (urination: $\mathrm{P}=0.03$; $\mathrm{UI} \mathrm{P}=0.01$ ); these differences were not observed in the control group (urination: $P=0.71$; UI: $P=0.68)$. The frequency of urination and $U I$ were lower in the electroacupuncture group than in the control group immediately after intervention (urination: $P=0.03$; UI: $P=0.03$ ). These differences remained after at least five months (intervention: urination: $\mathrm{P}=0.04 ; \mathrm{UI}: \mathrm{P}=0.01$; control: urination $\mathrm{P}=0.03$; $\mathrm{UI}: \mathrm{P}=$ $0.01)$; with differences lasting up to at least five months $(P<0.05$ for 
all outcomes). Formal comparisons were not conducted as suitable data were not received from study authors.

\section{Physical therapy interventions}

Booth 2016 reported no evidence of a difference in urgency between groups at weeks 12 and 26 (week 12: OR 0.49, 95\% Cl 0.13 to 1.87 ; week 26 : OR $0.68,95 \% \mathrm{Cl} 0.18$ to 2.59 ). The trial also reported no evidence of a difference in frequency between groups at weeks 12 and 26 (week 12: OR 0.45, 95\% Cl 0.11 to 1.84; week 26: OR $0.41,95 \% \mathrm{Cl} 0.12$ to 1.44 ). There was no evidence of an effect for nocturia at weeks 12 or 26 (week 12: OR 1.07, 95\% Cl 0.22 to 5.05; week 26: OR $0.73,95 \% \mathrm{Cl} 0.16$ to 3.32 ).

\section{Physical measures}

\section{Complementary therapy interventions}

One trial (66 participants) measured detrusor overactivity using maximum cystometric capacity and bladder compliance and detrusor leak point pressure (Liu 2013). However, data were reported as change from baseline in the intervention arm only and was not comparative. Therefore, the data were unusable.

\section{Health status and quality of life}

\section{Physical therapy interventions}

Data from one trial provided no evidence that transcutaneous posterior nerve stimulation made a difference to quality of life measured with the International Consultation on Incontinence Questionnaire - Lower Urinary Tract Symptoms Quality of Life questionnaire (ICIQLUTSqol) (MD 3.90, 95\% Cl -4.25 to $12.05 ; 30$ participants; Booth 2016; Analysis 2.4).

One trial reported quality of life using the EQ-5D-5L (Booth 2016). The EQ-5D-5L means were calculated as the average of the utilities at each time point of the trial. The calculation was done separately for each trial arm. Every participant's EQ-5D-5L response was translated into a utility score using the UK tariff and the mean utility was calculated across participants for each trial arm. The maximum possible score for a utility was 1 for a perfect health response in each of the five dimensions, the minimum was a negative number around -0.4 as reported in the UK tariff. There was no evidence of a difference between groups; both groups improved over time with a mean improvement of around $23 \%$. Baseline scores were 0.435 (SD 0.331 ) in the treatment group and 0.500 (SD 0.273) in the control group.

\section{Functional ability}

None of the trials reported functional ability.

\section{Participant satisfaction}

None of the trials reported participant satisfaction.

\section{Adverse events}

In Booth 2016, one (3.7\%) participant in the intervention group and one $(3.7 \%)$ participant in the sham group had residual urine volume of more than $150 \mathrm{~mL}$ at the six-week bladder scan. One participant (group unclear) had minor skin irritation and one participant (group unclear) reported ankle cramping. One trial reported no adverse events (Judge 1969). One trial reported no information on adverse events (Liu 2013).
Specific intervention versus another intervention (comparison 3)

Two trials with 76 participants compared a specific intervention with another intervention (Gelber 1997b; Zhang 1996).

Zhang 1996 reported on complementary therapy. Gelber 1997b compared a behavioural intervention (timed voiding) with a pharmacotherapy intervention (oxybutynin). However, the data were too few for useful analysis and no further data could be obtained from the investigators. This study was reported as ongoing in a conference abstract.

\section{Primary outcomes}

Number of participants continent after treatment

Complementary therapy interventions

One trial compared different acupuncture needles and depth of needle insertion to assess the effect on incontinence in 57 participants (Zhang 1996). The elongated needle was inserted to a depth of five Cun (Chinese inches; one of which is equivalent to the width of a thumb) and compared with a filiform needle inserted to a depth of $1.5 \mathrm{Cun}$. The study authors reported that, after four courses of treatment, $25(78.1 \%)$ participants in the elongated needle group were 'cured' (no incontinent episodes) versus 10 $(40 \%)$ in the filiform needle group.

\section{Number of incontinent episodes}

None of the trials reported the number of incontinent episodes.

\section{Perception of improvement or cure}

None of the trials reported perception of improvement or cure.

\section{Secondary outcomes}

Urinary symptoms

None of the trials reported urinary symptoms.

\section{Physical measures}

None of the trials reported physical measures.

\section{Health status and quality of life}

None of the trials reported health status or quality of life.

\section{Functional ability}

None of the trials reported functional ability.

\section{Participant satisfaction}

None of the trials reported participant satisfaction.

\section{Adverse events}

None of the trials reported information on adverse events.

\section{Combined intervention versus single intervention (comparison 4)}

One trial (23 participants) compared a combined intervention (sensory motor biofeedback plus timed prompted voiding) designed to promote urinary continence against a single intervention (timed voiding) (Lewis 1990). 


\section{Primary outcomes}

\section{Number of participants continent after treatment}

One trial compared a combined intervention (sensory motor biofeedback plus timed prompted voiding) designed to promote urinary continence against a single intervention (timed voiding) (Lewis 1990). There was no evidence that the combined intervention made a difference to the number of participants continent $(1 / 11(9 \%)$ in the combined intervention group versus $2 / 12(16.7 \%)$ in the control group; RR $0.55,95 \% \mathrm{Cl} 0.06$ to 5.21 ; Analysis 4.1.1).

\section{Number of incontinent episodes}

The combined intervention tested (sensory motor biofeedback plus timed prompted voiding versus timed prompted voiding) may have made a difference to the mean number of incontinent episodes; the control group reported fewer episodes of incontinence (3.5) compared with 5.7 in the combined group (MD 2.20, 95\% Cl 0.12 to 4.28; Lewis 1990; Analysis 4.2.1).

\section{Perception of improvement or cure}

The trial did not report perception of improvement or cure.

\section{Secondary outcomes}

\section{Urinary symptoms}

The trial did not report urinary symptoms.

\section{Physical measures}

The trial did not report physical measures.

\section{Health status and quality of life}

The trial did not report health status or quality of life.

\section{Functional ability}

The trial did not report functional ability.

\section{Participant satisfaction}

The trial did not report participant satisfaction.

\section{Adverse events}

The trial did not report any information on adverse events.

\section{Specific intervention versus attention control (comparison 5)}

One trial with 24 participants compared a specific intervention (TPTNS) with attention control (stretching exercises) (Monteiro 2014).

\section{Primary outcomes}

\section{Number of participants continent after treatment}

Data from one trial (24 participants) found no evidence that TPTNS made a difference to the number of participants continent after treatment compared to an attention control group undertaking stretching exercises (Monteiro 2014). The trial reported 4/12 $(33.3 \%)$ participants in the intervention group were continent on completion of the 45-day intervention period compared with 3/12 (25\%) participants in the attention control group (RR 1.33, 95\% Cl 0.38 to 4.72 ; Analysis 5.1.1).
One trial (24 participants) followed up participants for 12 months (Monteiro 2014). We were uncertain whether electrical stimulation of the posterior tibialis nerve improved urge incontinence: three (25\%) participants in the electrical stimulation group reported no urge incontinence compared with four (33\%) participants in the placebo group $(P=0.67)$.

\section{Number of incontinent episodes}

The trial did not report number of incontinent episodes.

\section{Perception of improvement or cure}

The trial did not report perception of improvement or cure postintervention (Monteiro 2014).

One study followed up participants for 12 months (Monteiro 2014). Electrical stimulation of the posterior tibial nerve may have made a difference to subjective improvement in symptoms (12 (100\%) participants in the electrical stimulation group versus three (25\%) participants in the placebo group; $P=0.001$ ).

\section{Secondary outcomes}

\section{Urinary symptoms}

Post-intervention, one study found seven (58\%) participants reported urinary urgency in the treatment group compared with 10 (83\%) participants in the attention control group $(P=0.18$; Monteiro 2014). Five (42\%) participants in the treatment group had nocturia post-intervention compared with nine $(75 \%)$ participants in the attention control group $(P=0.09)$.

One study (24 participants) followed up participants for 12 months (Monteiro 2014). We are uncertain whether electrical stimulation of the posterior tibialis nerve improved urinary urgency: six (50\%) participants in the electrical stimulation group reported urgency compared with nine $(75 \%)$ participants in the placebo group ( $P$ $=0.20$ ). Electrical stimulation of the posterior tibial nerve may have made a difference to nocturia at 12 months: one (8\%) participant in the TPTNS group had nocturia compared with six $(50 \%)$ participants in the attention control group $(P=0.02)$.

\section{Physical measures}

The trial did not report physical measures.

\section{Health status and quality of life}

The trial did not report health status or quality of life.

\section{Functional ability}

The trial did not report functional ability.

\section{Participant satisfaction}

The trial did not report participant satisfaction.

\section{Adverse events}

The trial did not report any information on adverse events.

\section{Subgroup analysis}

It was not possible to conduct planned subgroup analyses of the effect of urological diagnosis or time from stroke onset to recruitment to the trial due to lack of available data. 


\section{DISCUSSION}

\section{Summary of main results}

As there are many reasons why $\mathrm{UI}$ interventions might work differently in people with stroke compared with the general population, the review aimed to consider the effects (benefits and harms) of all interventions designed to treat $\mathrm{UI}$ after stroke in adults. Experimental studies tested a wide range of intervention types. Interventions were also variable within intervention categories, for example types of acupuncture (traditional or electroacupuncture) and areas of the body used for needle insertion.

\section{Behavioural interventions}

Five trials assessed behavioural interventions such as timed voiding and PFMT. All trials had very small sample sizes (fewer than 50 participants) and the limited published data for the main outcome of number of people continent after treatment did not permit meta-analysis. There was evidence from one trial that behavioural interventions may reduce the mean number of incontinent episodes in 24 hours (Tibaek 2005), while two trials suggest that PFMT may slightly improve mean daytime voiding frequency (Tibaek 2005; Tibaek 2017). However, behavioural interventions may make little or no difference to health status and quality of life (Shin 2016a; Tibaek 2005; low-quality evidence), urinary symptoms (night-time voiding frequency) (Tibaek 2005; Tibaek 2017), or physical measures (pelvic floor muscle function) compared with usual care (Tibaek 2005).

\section{Specialised professional input interventions}

There is no evidence that CNP intervention made a difference to urinary frequency or urgency at three months. The intervention may have improved participant satisfaction with the service at three months and the number of people continent at six months compared with usual care (Brittain 2000b; moderatequality evidence). The proportion of participants for whom results were available at six months was quite low (63\%) and some of the data could not be analysed.

\section{Complementary therapy interventions}

Complementary therapies using acupuncture, electroacupuncture and ginger-salt-partitioned moxibustion may increase the number of participants continent after treatment when compared with usual care (Chu 2011; Liu 2006; Zhang 2002; Zhou 1999; low-quality evidence), or placebo (Liu 2013), and may reduce mean daytime voiding frequency compared with usual care (Liu 2006). However, there is no evidence of a difference in night-time voiding frequency (Liu 2006; Song 2013).

These trials reported minimal methodological detail and it is likely they were of poor quality. It is also not clear whether, and how, these interventions are transferable to the health services of other countries. In addition, the newer studies reported the use of electroacupuncture while the earlier studies used traditional acupuncture. There was wide variation within the acupuncture interventions in terms of the target acupoint.

\section{Pharmacotherapy interventions}

In the small cross-over trial testing oral oestrogen against placebo, there were fewer incontinent episodes per week during oestrogen treatment in women with severe incontinence (Judge 1969). However, it is only known that the participants had a history of stroke. The high mean age of the participants in the trial, the presence of confusion and lack of mobility, combined with the setting in which the trial took place (two geriatric hospitals) suggested that continence problems may have been secondary to other conditions as well as stroke. For this reason, it is problematic to generalise these results to women after stroke. Furthermore, the dose prescribed was not in line with current recommendations. Hormone replacement therapy is now widely considered to be contraindicated in women who have had, or are at risk of, stroke and there is evidence that oestrogens increase incontinence in postmenopausal women (Hendrix 2005).

There is a lack of evidence on whether the many other forms of intervention shown to have some value in the general population would also be beneficial to people after stroke. In particular, there are no usable results from trials testing the use of bladder relaxants such as anticholinergics, programmes of timed voiding or bladder training, physical aids, environmental or lifestyle interventions.

\section{Physical therapy interventions}

Compared to usual care, physical therapy using transcutaneous electrical nerve stimulation may reduce the mean number of incontinent episodes in 24 hours (Guo 2014; Liu 2016a and Liu 2016b; low-quality evidence), reduce nocturia (Guo 2014), and probably improves functional ability (Liu 2016a and Liu 2016b; moderate-quality evidence). However, physical therapy interventions may make little or no difference to daytime voiding frequency (Guo 2014; Liu 2016a and Liu 2016b).

Compared to placebo, physical therapy makes little or no difference to health status, quality of life or the number of incontinent episodes (Booth 2016). Booth 2016 was a feasibility trial with 54 participants and was not designed to assess effectiveness.

\section{Overall completeness and applicability of evidence}

The major point of interest in the included studies was the mix of categories of intervention, including physical, behavioural and complementary therapies, drugs and professional input interventions such as using trained personnel and specific methods of managing care. However, within categories it was often not possible to combine studies due to data not being in the appropriate format. Sensitivity analyses were not conducted as too few studies reported outcomes of interest for each comparison type. It was not possible to conduct sensitivity analysis by low or high risk of bias as all except one of the studies were classified at unclear risk of bias for the domain of selective outcome reporting. The majority of studies had unclear risk of bias on the remainder of the risk of bias domains.

\section{Quality of the evidence}

Overall, the evidence was moderate or low-quality (see Summary of findings for the main comparison; Summary of findings 2; Summary of findings 3; Summary of findings 4). The most common reasons for downgrading the evidence were:

- risk of selection bias due to inadequate reporting of random sequence generation and allocation concealment; and

- imprecision due to small sample size. 
Only three trials had a sample of 100 participants or more (Brittain 2000b; Chu 2011; Song 2013); eight had fewer than 50 participants (Gelber 1997a; Gelber 1997b; Judge 1969; Lewis 1990; Monteiro 2014; Shin 2016a; Tibaek 2005; Tibaek 2017). Small sample sizes meant $\mathrm{Cls}$ were wide, making it difficult to ascertain if there were any clinically or statistically important differences between groups. The evidence may also be subject to small-study bias. Only four trials (reporting five comparisons) had adequate allocation concealment (Booth 2016; Brittain 2000b; Judge 1969; Liu 2016a and Liu 2016b); many were limited by poor reporting. For most, it was impossible to judge the extent to which they might have been prone to bias. The review was also limited by the lack of full data for five trials (Brittain 2000b; Chu 1997; Gelber 1997a; Gelber 1997b; Lewis 1990).

\section{Potential biases in the review process}

The method of dividing the sample size across comparisons with more than one treatment arm may not correct unit-of-analysis errors (Liu 2016a and Liu 2016b). Although this technique is in line with that described in the Cochrane Handbook for Systematic Reviews of Interventions, using a different method (combining data from multiple active treatment arms into a single arm) may have had an impact on Analysis 1.4.3 and Analysis 1.12.1, although the overall quantity of data available was small.

The original protocol for this review specified inclusion criteria for participants as having had a stroke in the previous 12 months. However, this definition proved unworkable as most of the trials did not report time since stroke. Time since stroke was less than three months in five studies reporting six comparisons (Chu 2011; Guo 2014; Liu 2016a and Liu 2016b; Song 2013; Tibaek 2017). One study reported the time since stroke as approximately six months (Shin 2016a). Booth 2016 had a variable time from stroke to recruitment $(12.2 \%$ (six) were recruited within three months of stroke; $20.4 \%$ (10) were between three and six months post-stroke; $12.2 \%$ (six) were between six and 12 months post-stroke and $55.1 \%$ (27) were recruited more than 12 months post-stroke). There was also a lack of clarity about whether UI was subsequent to stroke; only 12 trials specified that urinary continence problems were subsequent to stroke.

Very limited data were available for longer-term follow-up after the end of the intervention period. Where this was available, followup periods varied from three to 12 months. Six trials reported outcomes beyond the post-intervention period: Chu 2011, Guo 2014, Liu 2016a and Liu 2016b, Song 2013, Tibaek 2017 and Booth 2016 reported outcomes at three and six months. Liu 2013 did not report three-month data in a suitable format for inclusion in the review. Tibaek 2005, Tibaek 2017 and Brittain 2000b reported follow-up at six months (in the latter, follow-up data were missing for 85 (37\%) participants), and Monteiro 2014 at 12 months.

Duration of the intervention was also variable. Duration of PFMT versus usual care ranged from a six-week intervention consisting of three 50-minute sessions per week (Shin 2016a), to an intervention lasting 12 weeks (Tibaek 2005; Tibaek 2017). The duration of complementary therapy interventions ranged from one week (Chu 1997; Zhang 2002) to four weeks (Song 2013; Zhou 1999).

The extent to which the results can be generalised to people with continence problems solely as the result of stroke was unclear. Furthermore, the information regarding the severity of incontinence at the time of recruitment was unclear across the trials. It is very likely there were differences in participants' severity of incontinence based on examining the trials' inclusion criteria for 'incontinence'. Inclusion criteria detail for incontinence varied and ranged from participants with 'urinary incontinence' (Guo 2014); 'episodes occurring at least once a week' (Booth 2016); leakage several times a month or stress incontinence most of the time, frequency every half-hour/hour, nocturia three or more times a night and urgency most of the time (Brittain 2000b).

\section{Agreements and disagreements with other studies or reviews}

To our knowledge, there are no other comparable studies or reviews delivering interventions designed to improve continence after stroke in the rehabilitation phase of stroke recovery.

\section{AUTHORS' CONCLUSIONS}

\section{Implications for practice}

There is very little evidence from stroke-specific studies to guide practice. The lack of trials testing the same category of intervention means that recommendations for practice are based on the results of a few, usually small trials, providing generally low-quality evidence overall.

\section{Implications for research}

Evidence suggesting that beneficial outcomes may be achieved by structuring the management of care for people with urinary continence problems following a stroke points to the need for larger trials (Brittain 2000b). Given the variety of problems that can hinder the maintenance of continence after stroke, the use of individualised assessment and goal setting to tailor interventions to the neurological and functional problems of the individual would seem to be especially worthy of consideration.

Trials of complementary therapies, namely acupuncture alone (Chu 1997; Chu 2011; Liu 2006; Liu 2013; Song 2013; Zhang 1996; Zhang 2002; Zhou 1999), or combined with ginger-salt-partitioned moxibustion (Liu 2006), suggest these interventions may be worth investigating further with more rigorous study design.

Transcutaneous electrical nerve stimulation may reduce the number of incontinent episodes and warrants further investigation with the stroke population, particularly given its simplicity and ease of use (Booth 2016; Guo 2014; Liu 2016a and Liu 2016b).

Methods of managing continuing urinary incontinence (UI) such as behavioural interventions (e.g. bladder training, prompted voiding and pelvic floor muscle training) require testing with the stroke population, both in hospital and in the community post-discharge.

There is a need for more appropriately powered, multi-centre trials of interventions to provide robust evidence to improve UI after stroke. These trials may be simple (e.g. pharmacological interventions) but many will be complex interventions exploring behavioural interventions and more technical interventions, such as acupuncture or electrical stimulation.

Further research should use standardised definitions and classification systems to record details of the type and severity of stroke, as well as the type and severity of UI. Pre-stroke 
continence status, time since stroke and stroke recurrence should also be recorded, with clear inclusion criteria for continence status. Exclusion criteria should be given for comorbidities and clinical indicators of underlying urogenital or systemic conditions such as infection. Specific details of structured assessment and intervention protocols need to be given, with standardisation of treatment, measures of between groups contamination or differences, and tailoring of intervention to the early or later phases of rehabilitation. Outcome measures of UI and of urinary symptoms should be standardised with attention to their validity and reliability and the blinding of outcome assessment. The measurement of changes in health-related quality of life would be valuable. The time periods for review should be standardised for the early and later phases of rehabilitation. Lastly, sample size calculations and secure randomisation at either the cluster or individual participant level should be used appropriately.

\section{ACKNOWLEDGEMENTS}

For the 2019 update of this review, we are grateful to David Bousfield, Fiona Campbell, Nicola Dean and Richard Lindley for valuable comments on drafts of this review. We would also like to acknowledge the help of Sheila Wallace (Cochrane Incontinence) and Joshua Cheyne (Cochrane Stroke) for kindly updating the searches of their Cochrane Specialised Registers, and to Susan Wieland (Cochrane Complementary Medicine) for finding papers for this version of the review. We would particularly like to thank Lindsey Elstub, Eugenie Johnson and Luke Vale for all their advice and guidance.

For previous versions of the review, we would like to thank the authors of the 2008 review for their contributions (Stephen Cross, James Barrett, Beverley French, Michael Leathley, Christopher J Sutton). We also wish to thank members of the editorial team in the Incontinence Review Group, in particular Sheila Wallace and Cathryn Glazener, and Hazel Fraser and Brenda Thomas from the Stroke Review Group. Our thanks also go to the Faculty of Health and Wellbeing of the University of Central Lancashire for supporting this review, to trial authors for kindly responding to our requests for information and to the members of the Stroke Incontinence Interest Group for their valuable comments. Thank you to Gui Li of the Shanghai Medical Military University and Luyan Fang of the University of Central Lancashire for kindly translating some of our included studies in the earlier version of this review 


\section{R E F E R E N C E S}

\section{References to studies included in this review}

Booth 2016 \{published and unpublished data\}

* Booth J. Transcutaneous tibial nerve stimulation for REhabilitation And Treatment of Urinary Incontinence (TREATUI) after stroke: a feasibility study with pilot randomised controlled trial (Abstract). International Journal of Stroke 2016;11(Suppl 4):13. [NCT02239796; sr-incont75751]

Booth J, Thomas LH, Sutton CJ, Hagen S, McClurg D, Watkins $C L$, et al. A feasibility study with embedded randomised controlled pilot trial of transcutaneous posterior tibial nerve stimulation to alleviate stroke-related urinary incontinence: TREAT-UI [manuscript in final stages of preparation]. Manuscript to be submitted to: Trials 2019. [NCT02239796; sr-incont78112]

NCT02239796, Booth J. Feasibility controlled trial of tibial nerve stimulation for stroke related urinary incontinence (TREAT-UI) [A feasibility study for a randomised controlled trial of transcutaneous posterior tibial nerve stimulation to alleviate stroke-related urinary incontinence]. clinicaltrials.gov/ show/NCT02239796 (first received 15 September 2014). [NCT02239796; sr-incont64494]

\section{Brittain 2000b \{published data only\}}

Brittain KR, Potter JF. The treatment of urinary incontinence in stroke survivors (MS9). Report for NHS R\&D Programme on Cardiovascular Disease and Stroke Project, Division of Medicine for the Elderly, Department of Medicine, University of Leicester, in collaboration with the MRC Incontinence Study 2000. [srincont17214]

\section{Chu 1997 \{published data only\}}

Chu M, Feng J. Discussion on treating frequent urine due to multiple cerebral embolism with scalp acupuncture. Information on Traditional Chinese Medicine 1997;14(5):42. [srincont16940]

\section{Chu 2011 \{published data only\}}

Chu JM, Bao YH, Zou C, Zhao HL, Gong Y, Wang CM. Randomized controlled clinical trials for electroacupuncture treatment of urinary incontinence in stroke patients. Chen Tzu Yen Chiu Acupuncture Research 2011;36(6):428-32. [sr-incont44574]

\section{Gelber 1997a \{published data only\}}

Gelber DA, Swords L. Treatment of post-stroke urinary incontinence (abstract). Journal of Neurologic Rehabilitation 1997;11(2):131. [sr-incont10197]

\section{Gelber 1997b \{published data only\}}

Gelber DA, Swords L. Treatment of post-stroke urinary incontinence (abstract). Journal of Neurologic Rehabilitation 1997;11(2):131. [sr-incont10197]

\section{Guo 2014 \{published data only\}}

Guo ZF, Liu Y, Hu GH, Liu H, Xu YF. Transcutaneous electrical nerve stimulation in the treatment of patients with poststroke urinary incontinence. Clinical Interventions In Aging 2014;9:851-6. [sr-incont64865]
Judge 1969 \{published data only\}

Judge TG. The use of quinestradol in elderly incontinence women, a preliminary report. Gerontologica Clinica 1969;11(3):159-64. [sr-incont885]

Lewis 1990 \{published data only\}

Lewis AM, Travis ML, Gordon AL, Weaver HB, Reding MJ. Sensory-motor biofeedback for the treatment of urinary urge incontinence following stroke (Abstract). Clinical Research 1990;38(1):A10. [sr-incont20301]

Liu 2006 \{published data only\}

Liu H, Wang L. Randomized controlled study on ginger-saltpartitioned moxibustion at Shenque (CV 8) on urination disorders poststroke. Zhongguo Zhen Jiu [Chinese Acupuncture \& Moxibustion] 2006;26(9):621-4. [sr-incont22318]

Liu 2013 \{published data only\}

Liu Y, Liu L, Wang X. Electroacupuncture at points Baliao and Huiyang (BL35) for post-stroke detrusor overactivity. Neural Regeneration Research 2013;8(18):1663-72. [sr-incont61054]

\section{Liu 2016a \{published data only\}}

Liu Y, Xu G, Luo M, Teng HF. Effects of transcutaneous electrical nerve stimulation at two frequencies on urinary incontinence in poststroke patients: a randomized controlled trial. American Journal of Physical Medicine \& Rehabilitation 2016;95(3):183-93. [sr-incont70408]

\section{Liu 2016b \{published data only\}}

Liu Y, Xu G, Luo M, Teng HF. Effects of transcutaneous electrical nerve stimulation at two frequencies on urinary incontinence in poststroke patients: a randomized controlled trial. American Journal of Physical Medicine \& Rehabilitation 2016;95(3):183-93. [sr-incont70408]

\section{Monteiro 2014 \{published data only\}}

Monteiro ES, de Carvalho LB, Fukujima MM, Lora MI, do Prado GF. Electrical stimulation of the posterior tibialis nerve improves symptoms of poststroke neurogenic overactive bladder in men: a randomized controlled trial. Urology 2014;84(3):509-14. [sr-incont62527]

Shin 2016a \{published data only\}

Shin DC, Shin SH, Lee MM, Lee KJ, Song CH. Pelvic floor muscle training for urinary incontinence in female stroke patients: a randomized, controlled and blinded trial. Clinical Rehabilitation 2016;30(3):259-67. [sr-incont70429]

\section{Song 2013 \{published data only\}}

Song FJ, Jiang SH, Zheng SL, Ye TS, Zhang H, Zhu WZ, et al. Electroacupuncture for post-stroke urinary incontinence: a multi-center randomized controlled study. Zhongguo Zhen Jiu [Chinese Acupuncture \& Moxibustion] 2013;33(9):769-73. [srincont49433]

\section{Tibaek 2005 \{published and unpublished data\}}

Tibaek S, Gard G, Jensen R. Is there a long-lasting effect of pelvic floor muscle training in women with urinary 
incontinence after ischemic stroke? A 6-month follow-up study [erratum appears in Int Urogynecol J Pelvic Floor Dysfunct 2008;19(2):309]. International Urogynecology Journal 2007;18(3):281-7. [sr-incont22527]

* Tibaek S, Gard G, Jensen R. Pelvic floor muscle training is effective in women with urinary incontinence after stroke: a randomised, controlled and blinded study. Neurourology and Urodynamics 2005;24(4):348-57. [sr-incont20636]

Tibaek S, Jensen R, Lindskov G. Can quality of life be improved by pelvic floor muscle training in women with urinary incontinence after ischemic stroke? A randomised, controlled and blinded study. International Urogynecology Journal 2004;15(2):117-23. [sr-incont17409]

\section{Tibaek 2017 \{published data only\}}

NCT01042249, Tibaek S. Pelvic floor muscle training (PFMT) on lower urinary tract symptoms (LUTS) in men with stroke [The effect of pelvic floor muscle training on lower urinary tract symptoms (LUTS) in men with stroke]. clinicaltrials.gov/show/ NCT01042249 (first received 5 January 2010). [NCT01042249; srincont47881]

Tibaek S, Gard G, Dehlendorff C, Iversen HK, BieringSoerensen F, Jensen R. Can pelvic floor muscle training improve quality of life in men with mild to moderate post-stroke and lower urinary tract symptoms?. European Journal of Physical and Rehabilitation Medicine 2017;53(3):416-25. [sr-incont76787]

* Tibaek S, Gard G, Dehlendorff C, Iversen HK, BieringSoerensen $F$, Jensen $R$. Is pelvic floor muscle training effective for men with poststroke lower urinary tract symptoms? A singleblinded randomized, controlled trial. American Journal of Men's Health 2017;11(5):1460-71. [NTC01042249; sr-incont72233]

\section{Zhang 1996 \{published data only\}}

Zhang W-D, Xu N-G, Dong Y. Elongated-needle treatment of urinary incontinence caused by apoplexy. World Journal of Acupuncture-Moxibustion 1996;6(2):33-5. [sr-incont27388]

\section{Zhang 2002 \{published data only\}}

Zhang Z, Ma F, Ma Y. Observation on the effects of acupuncture in the treatment of urinary retention due to cerebral infarction in 36 patients. Heilongjiang Medicine and Pharmacy 2002;25(3):71. [sr-incont39297]

\section{Zhou 1999 \{published data only\}}

Zhou G, Wu D. 40 examples of using eye acupuncture and electriferous scalp acupuncture to treat urinary incontinence after cerebrovascular accident. Journal of Clinical Acupuncture 1999;15(9):33-4. [sr-incont24033]

\section{References to studies excluded from this review}

Byles 2006 \{published data only\}

ACTRN12606000511538, Byles J, Chiarelli P. Stroke incontinence study [A randomised controlled trial to evaluate the effects of a continence promotion program including real time ultrasound as biofeedback for pelvic floor muscle rehabilitation delivered to stroke survivors to reduce urinary incontinence and lower urinary tract symptoms]. www.anzctr.org.au/
ACTRN12606000511538.aspx (first received 7 December 2006). [sr-incont28048]

\section{Cook 1998 \{published data only\}}

Cook D, Huboky E, Hasskarl J, Hochrien S, Reding M. Effect of voiding position on urinary retention post stroke. Journal of Stroke and Cerebrovascular Diseases 1998;7(5):382.

\section{Engberg 2002 \{published and unpublished data\}}

Engberg S, Sereika SM, McDowell J, Weber E, Brodak I. Effectiveness of prompted voiding in treating urinary incontinence in cognitively impaired homebound older adults. Journal of Wound, Ostomy, and Continence Nursing 2002;29(5):252-65.

\section{EUCTR2009-009216-53-PT \{published data only\}}

EUCTR2009-009216-53-PT. A multicenter, long-term followup study of the safety and efficacy of two dose levels of BOTOX $^{\circledR}$ (botulinum toxin type A) purified neurotoxin complex in patients with urinary incontinence due to neurogenic detrusor overactivity. clinicaltrialsregister.eu/ctr-search/ trial/2009-009216-53/results (first received 6 March 2009). [EUCTR2009-009216-53-PT; NCT00876447; sr-incont40108; srincont64584]

\section{Gong 2013 \{published data only\}}

Gong J, Gong W. Bladder function training combined with water-drinking plan in the treatment of post-stroke patients with urinary incontinence. Modern Clinical Nursing 2013;12(4):49-52.

\section{Gousse 2007 \{published data only\}}

* Gousse A, Rivera R, Tunuguntla H, Barboglio P. Repeated intradetrusor injections of botulinum toxin-A in neurogenic overactive bladder: urodynamic, UDI-6 and quality of life data. Neurourology and Urodynamics 2007;26(5):730-1.

Gousse AE, Barboglio P, Rivera R, Tunuguntla H, Cason L. Repeat Botox $A$ injections and urodynamic findings in neurogenic $\mathrm{OAB}$ : long-term results of a single center prospective trial. Neurourology and Urodynamics 2007;26(7 Suppl):1071.

\section{Gross 1990 \{published data only\}}

Gross JC. Bladder dysfunction after a stroke: it's not always inevitable. Journal of Gerontological Nursing 1990;16(4):20-5, $41-2$.

\section{Humphreys 2014 \{published data only\}}

ISRCTN50857950, Humphreys G. Oxford Cognitive Screen (OCS)care: a study for developing and evaluating a care pathway for cognitive problems after stroke [A randomised controlled pilot study for developing and evaluating a care pathway for cognitive problems after stroke (the OCS-case project)]. isrctn.com/ISRCTN50857950 (first received 14 February 2014).

\section{ISRCTN97151578 \{published data only\}}

ISRCTN97151578. The efficacy of acupuncture and moxibustion in urination disorders after stroke [The effect of both acupuncture and moxibustion versus standard Western therapy in reducing the rate of deformity of stroke and in improving the symptoms of urination disorders after stroke in adult 
patients: a multicentre randomised controlled trial]. isrctn.com/ ISRCTN97151578 (first received 24 September 2010). [srincont77896]

\section{Kim 2003 \{published data only\}}

Kim JH, Rivas DA, Shenot PJ, Green B, Kennelly M, Erickson JR, et al. Intravesical resiniferatoxin for refractory detrusor hyperreflexia: a multicenter, blinded, randomized, placebo-controlled trial. Journal of Spinal Cord Medicine 2003;26(4):358-63.

\section{Kuo 2007 \{published data only\}}

Kuo H-C. Comparison of the therapeutic effects of urethral injections of 50 and 100 units of botulinum A toxin for voiding dysfunction. Tzu Chi Medical Journal 2007;19(3):134-8.

\section{Madersbacher 2004 \{published data only\}}

Madersbacher H, Menarini M, Dorsam J, Sember ME, Chiang H. Investigation on an individually adjusted oral trospium chloride (TC) therapy in patients suffering from neurogenic overactive bladder. Joint Meeting of the International Continence Society (ICS) (34th Annual Meeting) and the International UroGynecological Association (IUGA); 2004 Aug 23-27; Paris, France. 2004:Abstract number 581.

\section{Madersbacher 2005 \{published data only\}}

Madersbacher H, Hessdoerfer E, Martan A, Nurkiewicz W, Bruenjes R, Shah J. Safety, tolerability and efficacy of propiverine long-term treatment. International Continence Society (ICS), 35th Annual Meeting; 2005 Aug 28-Sep 2; Montreal, Canada. 2005:Abstract number 573.

\section{McDowell 1999 \{published and unpublished data\}}

McDowell BJ, Engberg S, Sereika S, Donovan N, Jubeck ME, Weber $E$, et al. Effectiveness of behavioral therapy to treat incontinence in homebound older adults. Journal of the American Geriatrics Society 1999;47(3):309-18.

\section{Moon 2012 \{published data only\}}

* Moon HJ, Chun MH, Lee SI, Kim DH. The usefulness of bladder reconditioning before indwelling urethral catheter removal from stroke patients. American Journal of Physical Medicine \& Rehabilitation 2012;918(8):681-8.

Moon HJ, Chun MH, Lee SI, Kim DH, Lee SJ, Yun GJ, et al. The usefulness of bladder reconditioning before indwelling urethral catheter removal from stroke patients (Abstract number 242, Poster number 227). Neurorehabilitation and Neural Repair 2012; Vol. 626, issue 6:743.

\section{NCT00213577 \{published data only\}}

Grise P, Ruffion A, Chartier-Kastler E, Denys P, Egon G. Impact on continence of only bladder botulinum toxin injection without anticholinergics in neurogenic patients (Abstract number 356). 38th Annual Meeting of the International Continence Society (ICS); 2008 Oct 20-24; Cairo, Egypt. 2008.

* Grise P, Ruffion A, Denys P, Egon G, Chartier KE. Efficacy and tolerability of botulinum toxin type $A$ in patients with neurogenic detrusor overactivity and without concomitant anticholinergic therapy: comparison of two doses. European Urology 2010;58(5):759-66.
NCT00213577, Grise P. NEUROTOX: two dosages of type A botulinic toxin in neurogenic overactive bladder [Evaluation of the efficacy and tolerance of 2 dosages of type A botulinic toxin in neurogenic are active bladder resistant to oral medical treatment]. clinicaltrials.gov/show/NCT00213577 (first received 21 September 2005). [sr-incont64452]

\section{NCT01275261 \{published data only\}}

NCT01275261, Poisson SN. Pilot randomized trial of a nursing protocol to avoid bladder catheterization in patients with stroke. clinicaltrials.gov/show/NCT01275261 (first received 12 January 2011). [sr-incont60939]

\section{Sakakibara 2008 \{published data only\}}

Sakakibara R, Ito T, Uchiyama T, Awa Y, Yamaguchi C, Hattori T. Effects of milnacipran and paroxetine on overactive bladder due to neurologic diseases: a urodynamic assessment. Urologia Internationalis 2008;81(3):335-9.

\section{Shin 2016c \{published data only\}}

NCT02472288, Lee E-J. Electroacupuncture on poststroke urinary retention [Randomised controlled trial of electroacupuncture versus sham electroacupuncture for urinary retention of poststroke patients: a study protocol]. clinicaltrials.gov/show/NCT02472288 (first received 15 June 2015). [sr-incont69692]

* Shin S, Lee J, Yoo J, Lim SM, Lee E. Electroacupuncture versus sham electroacupuncture for urinary retention in poststroke patients: study protocol for a multicenter, randomized controlled trial. Trials 2016;17:197. [DOI: 10.1186/ s13063-016-1315-3; NCT02472288; sr-incont74143]

\section{Smilskalne 2009 \{published data only\}}

Smilskalne B, Berzina G, Gormalova J, Vetra A. Post-stroke urinary incontinence. Effectiveness of management. International Journal of Rehabilitation Research. Internationale Zeitschrift fur Rehabilitationsforschung. Revue Internationale de Recherches de Readaptation 2009;32(Suppl 1):S111.

\section{Stohrer 2013 \{published data only\}}

Stohrer M, Murtz G, Kramer G, Warnack W, Primus G, Jinga V, et al. Efficacy and tolerability of propiverine hydrochloride extended-release compared with immediate-release in patients with neurogenic detrusor overactivity. Spinal Cord 2013;51(5):419-23.

\section{Tekeoglu 1998 \{published data only\}}

Tekeoglu Y, Adak B, Goksoy T. Effect of transcutaneous electrical nerve stimulation (TENS) on Barthel Activities of Daily Living (ADL) Index score following stroke. Clinical Rehabilitation 1998;12(4):277-80.

\section{Thomas 2011 \{published data only\}}

Thomas LH, Watkins CL, French B, Sutton C, Forshaw D, Cheater F, et al. Study protocol: ICONS: identifying continence options after stroke: a randomised trial. Trials 2011;12:131.

\section{Vinsnes 2010 \{published data only\}}

Vinsnes AG, Helbostad JL, Nyronning S, Granbo R, Seim A. A general physical training programme for residents in 
nursing homes and the effect on urinary incontinence: a randomized controlled trial. Neurourology and Urodynamics 2010;29(6):852-3.

\section{Wikander 1998 \{published data only\}}

Wikander B, Ekelund P, Milsom I. An evaluation of multidisciplinary intervention governed by functional independence measure (FIMSM) in incontinent stroke patients. Scandinavian Journal of Rehabilitation Medicine 1998;30(1):15-21.

Yun 2007 \{published data only\}

Yun SP, Jung WS, Park SU, Moon SK, Park JM, Ko CN, et al. Effects of moxibustion on the recovery of post-stroke urinary symptoms. American Journal of Chinese Medicine 2007;35(6):947-54.

Zhu 2003 \{published data only\}

Zhu Y, Zhu X, Zhu D, Jin Z. Meclofenoxate in treating urinary incontinence after acute cerebral infarction. Zhongguo Xin Yao Yu Lin Chuang Za Zhi [Chinese Journal of New Drugs and Clinical Remedies] 2003;22(9):520-2. [ISSN 1007-7669]

\section{References to studies awaiting assessment}

\section{ACTRN12617000162314 \{published data only\}}

ACTRN12617000162314, Ptaszkowski K. Electromyographic evaluation of the effect of pelvic floor muscle training to improve the function of the lower urinary tract in women with ischemic stroke [Electromyographic evaluation of the effect of pelvic floor muscle training to improve the function of the lower urinary tract in women with ischemic stroke: a prospective, randomized study with a control group]. anzctr.org.au/ ACTRN12617000162314.aspx (first received 24 November 2016). [ACTRN12617000162314; sr-incont76202]

\section{ChiCTR-INR-16010239 \{published data only\}}

ChiCTR-INR-16010239, Yang Y. Treating urinary retention after stroke with acupuncture: a clinical trial. chictr.org.cn/ showproj.aspx?proj=17368 (first received 23 December 2016). [ChiCTR-INR-16010239; sr-incont76233]

\section{Wang 2014 \{published data only\}}

ISRCTN44706974, Wang L, Liu H. Evaluation of moxibustion therapy for urge urinary incontinence after stroke [Evaluation of moxibustion therapy for urge urinary incontinence after stroke: a multicenter, randomized, single-blinded, controlled clinical trial]. isrctn.com/ISRCTN44706974 (first received 5 December 2013). [sr-incont64520]

* Wang L, Wang L, Shi G, Zeng L, Yang Y, Zhang T, et al. Efficacy and safety of ginger-salt-indirect moxibustion for urge urinary incontinence after stroke: protocol for a pilot multicentre randomised controlled trial. BMJ Open 2014;4(10):e006326. [ISRCTN44706974; sr-incont63970]

\section{References to ongoing studies \\ NCT02568774 \{published data only\}}

NCT02568774, Chen H, Yeung WF. Acupuncture on post-stroke overactive bladder [Acupuncture on post-stroke overactive bladder: a randomized controlled trial]. clinicaltrials.gov/show/ NCT02568774 (first received 6 October 2015). [NCT02568774; srincont71354]

Shin 2016b \{published data only\}

NCT02819336, Lee E-J. Effectiveness and safety of electroacupuncture on poststroke urinary incontinence [Effectiveness and safety of electroacupuncture on poststroke urinary incontinence: a pilot multicentered, randomized, parallel, sham-controlled trial]. clinicaltrials.gov/show/ NCT02819336 (first received 30 June 2016). [NCT02819336; srincont73332]

Shin S, Lee J, Yu A, Yoo J, Lee E. Effectiveness and safety of electroacupuncture on poststroke urinary Incontinence: study protocol of a pilot multicentered, randomized, parallel, shamcontrolled trial. Evidence-based Complementary and Alternative Medicine: ECAM 2016;2016:Article ID 5709295. [NCT02819336; srincont74325]

\section{Additional references}

\section{Abrams 2002}

Abrams P, Cardozo L, Fall M, Griffiths D, Rosier P, Ulmsten U, et al. The standardisation of terminology of lower urinary tract function: report from the Standardisation Sub-committee of the International Continence Society. Neurourology and Urodynamics 2002;21(2):167-78.

\section{Al-Shaikh 2018}

Al-Shaikh G, Syed S, Osman S, Bogis A, Al-Badr A. Pessary use in stress urinary incontinence: a review of advantages, complications, patient satisfaction, and quality of life. International Journal of Women's Health 2018;10:195-201. [DOI: 10.2147/IJWH.S152616]

\section{Alhasso 2005}

Alhasso A, Glazener CMA, Pickard R, N'Dow JMO. Adrenergic drugs for urinary incontinence in adults. Cochrane Database of Systematic Reviews 2005, Issue 3. [DOI: 10.1002/14651858.CD001842.pub2]

\section{Arkan 2018}

Arkan G, Beser A, Ozturk V. Experiences related to urinary incontinence of stroke patients: a qualitative descriptive study. Journal of Neuroscience Nursing 2018;50(1):42-7.

\section{Ayeleke 2015}

Ayeleke RO, Hay-Smith EJC, Omar MI. Pelvic floor muscle training added to another active treatment versus the same active treatment alone for urinary incontinence in women. Cochrane Database of Systematic Reviews 2015, Issue 11. [DOI: 10.1002/14651858.CD010551.pub3] 


\section{Barer 1989}

Barer DH. Continence after stroke: useful predictor or goal of therapy?. Age and Ageing 1989;18(3):183-91.

\section{Barrett 2002}

Barrett JA. Bladder and bowel problems after a stroke. Reviews in Clinical Gerontology 2002;12(3):253-67.

\section{Brittain 1998a}

Brittain KR, MRC Continence Programme, R\&D Stroke and Incontinence Study. Urinary symptoms and depression in stroke survivors (Abstract number 116). Age and Ageing 1998;27(Suppl 1):P72-3.

\section{Brittain 1998b}

Brittain KR, Peet SM, Castleden MD. Stroke and incontinence. Stroke 1998;29(2):524-8.

\section{Brittain 1999}

Brittain KR, Peet SM, Potter JF, Castleden CM. Prevalence and management of urinary incontinence in stroke survivors. Age and Ageing 1999;28(6):509-11.

\section{Brittain 2000a}

Brittain KR, Perry SI, Peet SM, Shaw C, Dallosso H, Assassa RP, et al. Prevalence and impact of urinary symptoms among community-dwelling stroke survivors. Stroke 2000;31(4):886-91.

\section{Brittain 2007}

Brittain KR, Shaw C. The social consequences of living with and dealing with incontinence--a carers perspective. Social Science \& Medicine 2007;65(6):1274-83.

\section{Burney 1996a}

Burney TL, Senapati M, Desai S, Choudary ST, Badlani GH. Effects of cerebrovascular accident on micturition. Urologic Clinics of North America 1996;23(3):483-90.

\section{Burney 1996b}

Burney TL, Senepati M, Desai S, Choudhary MS, Badlani GH. Acute cerebrovascular accident and lower urinary tract dysfunction: a prospective correlation of the site of brain injury with urodynamic findings. Journal of Urology 1996;156(5):1748.

\section{Cody 2012}

Cody JD, Jacobs ML, Richardson K, Moehrer B, Hextall A. Oestrogen therapy for urinary incontinence in post-menopausal women. Cochrane Database of Systematic Reviews 2012, Issue 10. [DOI: 10.1002/14651858.CD001405.pub3]

\section{DerSimonian 1986}

DerSimonian R, Laird N. Meta-analysis in clinical trials. Controlled Clinical Trials 1986;7(3):177-88.

\section{Eustice 2000}

Eustice S, Roe B, Paterson J. Prompted voiding for the management of urinary incontinence in adults. Cochrane Database of Systematic Reviews 2000, Issue 2. [DOI: 10.1002/14651858.CD002113]

\section{Freedland 2011}

Freedland KE, Mohr DC, Davidson KW, Schwartz JE. Usual and unusual care: existing practice control groups in randomised controlled trials of behavioural interventions. Psychosomatic Medicine 2011;73(4):323-35.

\section{Guyatt 2008}

Guyatt GH, Oxman AD, Vist G, Kunz R, Falck-Ytter Y, et al. GRADE Working Group. Rating quality of evidence and strength of recommendations: GRADE: an emerging consensus on rating quality of evidence and strength of recommendations. BMJ 2008;336(7650):924-6.

\section{Hay-Smith 2011}

Hay-Smith EJC, Herderschee R, Dumoulin C, Herbison GP. Comparisons of approaches to pelvic floor muscle training for urinary incontinence in women. Cochrane Database of Systematic Reviews 2011, Issue 12. [DOI: 10.1002/14651858.CD009508]

\section{Hendrix 2005}

Hendrix SL, Cochrane BB, Nygaard IE, Handa VL, Barnabei VM, Iglesia $C$, et al. Effects of estrogen with and without progestin on urinary incontinence. JAMA 2005;293(8):935-48.

\section{Higgins 2011}

Higgins JP, Altman DG, Gøtzsche PC, Jüni P, Moher D, Oxman AD, et al. The Cochrane Collaboration's tool for assessing risk of bias in randomised trials. BMJ 2011;343:d5928.

\section{Imamura 2015}

Imamura M, Williams K, Wells M, McGrother C. Lifestyle interventions for the treatment of urinary incontinence in adults. Cochrane Database of Systematic Reviews 2015, Issue 12. [DOI: 10.1002/14651858.CD003505.pub5]

\section{Intercollegiate Stroke Working Party 2016}

Royal College of Physicians Intercollegiate Stroke Working Party. National Clinical Guideline for Stroke. 5th Edition. London: Royal College of Physicians, October 2016. Available at: rcplondon.ac.uk/guidelines-policy/stroke-guidelines (accessed 18 June 2018).

\section{International Continence Society 2015}

International Continence Society. Fact sheets: a background to urinary and faecal incontinence. Bristol, UK: International Continence Society, August 2015. Available at: ics.org/public (accessed 28 March 2018).

\section{ISWP 2016}

Intercollegiate Stroke Working Party. National Clinical Guidelines for Stroke. London: Royal College of Physicians 2016.

\section{Ju 2017}

Ju ZY, Wang K, Cui HS, Yao Y, Liu SM, Zhou J, et al. Acupuncture for neuropathic pain in adults. Cochrane Database of Systematic Reviews 2017, Issue 12. [DOI: 10.1002/14651858.CD012057.pub2] 


\section{Kolominsky-Rabas 2003}

Kolominsky-Rabas PL, Hilz MJ, Neundoerfer B, Heuschmann PU. Impact of urinary incontinence after stroke: results from a prospective population-based stroke register. Neurourology and Urodynamics 2003;22(4):322-7.

\section{Kuteesa 2006}

Kuteesa W, Moore KH. Anticholinergic drugs for overactive bladder. Australian Prescriber 2006;29(1):22-4.

\section{Lim 2015}

Lim SM, Yoo J, Lee E, Kim HJ, Shin S, Han G, et al. Acupuncture for spasticity after stroke: a systematic review and metaanalysis of randomized controlled trials. Evidencebased Complementary and Alternative Medicine: ECAM 2015;2015:Article ID 870398. [DOI: 10.1155/2015/870398]

\section{Limampai 2017}

Limampai P, Wongsrithep W, Kuptniratsaikul V. Depression after stroke at 12-month follow-up: a multicenter study. International Journal of Neuroscience 2017;127(10):887-92.

\section{Mantel 1959}

Mantel N, Haenszel W. Statistical aspects of the analysis of data from retrospective studies of disease. Journal of the National Cancer Institute 1959;22(4):719-48.

\section{Marinkovic 2001}

Marinkovic S, Badlani G. Voiding and sexual dysfunction after cerebrovascular accidents. Journal of Urology 2001;165(2):359-70.

\section{Meijer 2003}

Meijer R, Ihnenfeldt DS, de Groot IJM, van Limbeek J, Vermeulen M, de Haan RJ. Prognostic factors for ambulation and activities of daily living in the subacute phase after stroke. A systematic review of the literature. Clinical Rehabilitation 2003;17(2):119-29.

\section{Nabi 2006}

Nabi G, Cody JD, Ellis G, Hay-Smith J, Herbison GP. Anticholinergic drugs versus placebo for overactive bladder syndrome in adults. Cochrane Database of Systematic Reviews 2006, Issue 4. [DOI: 10.1002/14651858.CD003781.pub2]

\section{Nakayama 1997}

Nakayama H, Jorgensen HS, Pedersen PM, Raaschou HO, Olsen TS. Prevalence and risk factors of incontinence after stroke. The Copenhagen Stroke Study. Stroke 1997;28(1):58-62.

\section{NICE 2012}

National Clinical Guideline Centre, National Institute for Health and Care Excellence (NICE). Urinary Incontinence in Neurological Disease: assessment and management. Clinical Guidelines 148 (CG148). Manchester, UK: National Institute for Health and Care Excellence (NICE), 2012. Available at: nice.org.uk/guidance/cg148 (accessed 18 June 2018).

\section{Ostaszkiewicz 2004a}

Ostaszkiewicz J, Johnston L, Roe B. Habit retraining for the management of urinary incontinence in adults.
Cochrane Database of Systematic Reviews 2004, Issue 2. [DOI: 10.1002/14651858.CD002801.pub2]

\section{Ostaszkiewicz 2004b}

Ostaszkiewicz J, Johnston L, Roe B. Timed voiding for the management of urinary incontinence in adults. Cochrane Database of Systematic Reviews 2004, Issue 1. [DOI: 10.1002/14651858.CD002802.pub2]

\section{Paik 2013}

Paik SH, Han SR, Kwon OJ, Ahn YM, Lee BC, Ahn SE. Acupuncture for the treatment of urinary incontinence: a review of randomized controlled trials. Experimental and Therapeutic Medicine 2013;6(3):773-80.

\section{Patel 2001}

Patel M, Coshall C, Lawrence E, Rudd AG, Wolfe CD. Recovery from poststroke urinary incontinence: associated factors and impact on outcome. Journal of the American Geriatrics Society 2001;49(9):1229-33.

\section{Pettersen 2006}

Pettersen R, Wyller TB. Prognostic significance of micturition disturbances after acute stroke. Journal of the American Geriatrics Society 2006;54(12):1878-84.

\section{Reference Manager 2012 [Computer program]}

Thomson Reuters. Reference Manager Professional Edition Version 12. New York: Thomson Reuters, 2012.

\section{Review Manager 2014 [Computer program]}

Copenhagen: The Nordic Cochrane Centre, The Cochrane Collaboration. Review Manager (RevMan). Version 5.3. Copenhagen: The Nordic Cochrane Centre, The Cochrane Collaboration, 2014

\section{Roe 2007}

Roe B, Ostaszkiewicz J, Milne J, Wallace S. Systematic reviews of bladder training and voiding programmes in adults: a synopsis of findings from data analysis and outcomes using metastudy techniques. Journal of Advanced Nursing 2007;57(1):15-31.

\section{Shefchyk 2001}

Shefchyk SJ. Sacral spinal interneurones and the control of urinary bladder and urethral striated sphincter muscle function. Journal of Physiology 2001;533(Pt 1):57-63.

\section{Song 2011}

Song FJ, Zhang H, Zheng SL, Fang JH, Liu HF. Research review on apoplectic urinary incontinence treated with acupuncturemoxibustion in recent 5 years. Zhongguo Zhen Jiu [Chinese Acupuncture \& Moxibustion] 2011;31(10):957-60.

\section{Staskin 2012}

Staskin D, Peters K, MacDiarmid S, Shore N, de Groat WC. Percutaneous tibial nerve stimulation: a clinically and cost effective addition to the overactive bladder algorithm of care. Current Urology Reports 2012;13(5):327-34. 


\section{Talwar 1993}

Talwar A, Badlani GH. Urologic problems in cerebrovascular accidents. Problems in Urology 1993;7:41. [ISSN 0889-471X]

\section{Tseng 2015}

Tseng CN, Huang GS, Yu PJ, Lou MF. A qualitative study of family caregiver experiences of managing incontinence in stroke survivors. PloS One 2015;10(6):e0129540.

\section{VanderPloeg 2009}

VanderPloeg K, Yi X. Acupuncture in modern society. Journal of Acupuncture and Meridian Studies 2009;2(1):26-33.

\section{Ween 1996}

Ween JE, Alexander MP, D'Esposito M, Roberts M. Incontinence after stroke in a rehabilitation setting: outcome associations and predictive factors. Neurology 1996;47(3):659-63.

\section{WHO 1989}

World Health Organization. WHO Task Force on Stroke and other Cerebrovascular Disorders: Recommendation on stroke prevention, diagnosis and therapy. Stroke 1989;20:1407-31.

\section{Williams 1993}

Williams A. Caregivers of persons with stroke: their physical and emotional well-being. Quality of Life Research 1993;2(3):213-20.

\section{Williams 2012}

Williams MP, Srikanth V, Bird M, Thrift AG. Urinary symptoms and natural history of urinary continence after first-ever stroke--a longitudinal population-based study. Age and Ageing 2012;41(3):371-6.

\section{References to other published versions of this review Thomas 2003}

Thomas LH, Barrett J, Cross S, French B, Leathley M, Legg L, et al. Prevention and treatment of urinary incontinence after stroke in adults. Cochrane Database of Systematic Reviews 2003, Issue 4. [DOI: 10.1002/14651858.CD004462]

\section{Thomas 2005}

Thomas LH, Barrett J, Cross S, French B, Leathley M, Sutton C, et al. Prevention and treatment of urinary incontinence after stroke in adults. Cochrane Database of Systematic Reviews 2005, Issue 3. [DOI: 10.1002/14651858.CD004462.pub2]

\section{Thomas 2008}

Thomas LH, Cross S, Barrett J, French B, Leathley M, Sutton CJ, et al. Treatment of urinary incontinence after stroke in adults. Cochrane Database of Systematic Reviews 2008, Issue 1. [DOI: 10.1002/14651858.CD004462.pub3]

* Indicates the major publication for the study

\section{CHARACTERISTICS OF STUDIES}

Characteristics of included studies [ordered by study ID]

Booth 2016

Methods Design: randomised controlled feasibility trial

Study dates: May 2014 to August 2016

Setting: UK, Scotland; intervention and sham intervention delivered in participants' homes.

Participants 54 men and women

Number and \% women: intervention 15 (55.6\%); control 15 (55.6\%)

Age (mean): intervention 67.1 (SD 10.9) years; control 66.9 (SD 11.4) years

Description of stroke and UI:

Ischaemic stroke: intervention 21 (87.5\%); control $22(88.0 \%)$

Haemorrhagic stroke: intervention 3 (12.5\%); control 2 (8.0\%)

Inclusion criteria: men or women aged $\geq 18$ years with diagnosis of stroke based on the WHO criteria (WHO 1989) and stroke-related UI of at least once weekly. Onset of stroke $\geq 4$ weeks previously and discharged from hospital to own home within previous 6 months. Physically, cognitively and communicatively able and willing to learn to apply TPTNS or has carer able and willing to learn. Informed consent provided (by carer proxy if required).

Exclusion criteria: pre-existing UI prior to stroke, current UTI or voiding dysfunction requiring intermittent/indwelling catheterisation, cardiac pacemaker in situ, concurrent neurological diagnosis including 
Booth 2016 (Continued)

dementia and multiple sclerosis, diabetic neuropathy, reduced/absent sensation at electrode placement sites, postvoid residual urine volume $>150 \mathrm{~mL}$, unable to provide informed consent.

Interventions

Intervention group: 27 participants. TPTNS intervention programme self-delivered or delivered by the stroke survivor's carer in the stroke survivor's home following individual education to enable this to take place. The full TPTNS treatment programme comprised 12 stimulation sessions of 30 minutes' duration, delivered twice weekly over 6 weeks. Two surface electrodes applied to non-hemiparetic ankle, where appropriate, or right ankle where no hemiparesis exists: first electrode placed $6 \mathrm{~cm}$ cephalad to medial malleolus and $2 \mathrm{~cm}$ behind tibia. Second electrode placed $10 \mathrm{~cm}$ cephalad to first. Electrical stimulator cathode lead connected to distal electrode and anode lead to proximal. Electrical stimulator preprogrammed to safely deliver 30 minutes of continuous stimulation with a pulse frequency of $10 \mathrm{~Hz}$ and pulse width $200 \mu \mathrm{s}$. Intensity of the current depends on the stroke survivor's perception threshold and individual comfort and is self-adjusted at each session, but will normally range between $15 \mathrm{~mA}$ and $40 \mathrm{~mA}$.

Control group: 27 participants. Sham stimulation group received a similar programme of $12 \times 30$ minute sessions twice weekly for 6 weeks. However, surface electrodes were positioned on the lateral malleolar area of ankle, not the medial aspect, to avoid the posterior tibial nerve. Stimulation intensity increased until sensation was reported, then turned down to $4 \mathrm{~mA}$ for the 30-minute session, ensuring that despite avoiding the posterior tibial nerve, there was no therapeutic stimulation provided.

All participants, regardless of group, were informed that they may experience little or no sensation during the treatment session.

Outcomes
Primary outcome: reported episodes and severity of episodes of UI (ICIQ-UI-SF)
$\begin{aligned} & \text { Secondary outcomes: number of adverse events, urinary symptoms (AUA Symptom Index), functional } \\ & \text { ability (Barthel Index), quality of life (EQ-5D-5L; ICIQLUTSqol) }\end{aligned}$

\begin{tabular}{|c|c|c|}
\hline Funding source & \multicolumn{2}{|l|}{ Stroke Association } \\
\hline $\begin{array}{l}\text { Declarations of inter- } \\
\text { est among primary re- } \\
\text { searchers }\end{array}$ & \multicolumn{2}{|l|}{ None reported } \\
\hline Notes & \multicolumn{2}{|l|}{ No baseline imbalance } \\
\hline \multicolumn{3}{|l|}{ Risk of bias } \\
\hline Bias & Authors' judgement & Support for judgement \\
\hline $\begin{array}{l}\text { Random sequence genera- } \\
\text { tion (selection bias) }\end{array}$ & Low risk & $\begin{array}{l}\text { Randomisation stratified by sex; block length used was random to help main- } \\
\text { tain allocation concealment. }\end{array}$ \\
\hline $\begin{array}{l}\text { Allocation concealment } \\
\text { (selection bias) }\end{array}$ & Low risk & $\begin{array}{l}\text { Randomisation delivered using 'SealedEnvelope.com' using an allocation list } \\
\text { derived by Lancashire CTU staff. Trial staff recruited participants. For consent- } \\
\text { ed participants, after having checked eligibility, recruiting staff entered par- } \\
\text { ticipant's information (sex) into 'SealedEnvelope.com' (online) and were in- } \\
\text { formed by email of the participant's allocation (to TPTNS or sham). }\end{array}$ \\
\hline $\begin{array}{l}\text { Blinding of participants } \\
\text { and personnel (perfor- } \\
\text { mance bias) } \\
\text { All outcomes }\end{array}$ & Low risk & $\begin{array}{l}\text { Participants blind as neither intervention nor sham group knew their alloca- } \\
\text { tion. TPTNS education provided by study research associates who could not } \\
\text { be blinded to group allocation as procedure for each group was different. Out- } \\
\text { come measurement was unlikely to be influenced by lack of blinding of per- } \\
\text { sonnel. }\end{array}$ \\
\hline
\end{tabular}


Booth 2016 (Continued)

Blinding of outcome as- Low risk Outcome measures were self-reported using postal questionnaires. Where sessment (detection bias) telephone completion was necessary, this was done by a researcher blind to All outcomes group allocation.

\begin{tabular}{|c|c|c|}
\hline $\begin{array}{l}\text { Incomplete outcome data } \\
\text { (attrition bias) } \\
\text { All outcomes }\end{array}$ & Unclear risk & $\begin{array}{l}\text { Of the } 54 \text { participants recruited, } 44(81.5 \%) \text { completed the } 6 \text {-week outcomes, } \\
40(74.1 \%) \text { completed the } 12 \text {-week outcomes and } 39(72.2 \%) \text { completed the } \\
26 \text {-week outcomes. Reasons for missing data provided. }\end{array}$ \\
\hline
\end{tabular}

\begin{tabular}{|c|c|c|}
\hline $\begin{array}{l}\text { Selective reporting (re- } \\
\text { porting bias) }\end{array}$ & Low risk & $\begin{array}{l}\text { Protocol available. Some prespecified secondary outcomes (e.g. Barthel Index, } \\
\text { Modified Rankin Scale, postvoid residual urine volume) not reported in final re- } \\
\text { port but supplied by study author. }\end{array}$ \\
\hline
\end{tabular}

\begin{tabular}{ll}
\hline Other bias $\quad$ Low risk & $\begin{array}{l}\text { Quote: "The groups were similar in their baseline characteristics indicating the } \\
\text { success of randomisation." }\end{array}$
\end{tabular}

Brittain 2000b

\begin{tabular}{ll}
\hline Methods & Design: RCT \\
& Study dates: August 1997 to June 2000 \\
& Setting: UK, at home \\
\hline Participants & 232 male $(61 \%)$ and female (39\%) adult stroke survivors with UI. Gender by group allocation not report- \\
ed. & Age: $40-96$ years, mean 70 years \\
Inclusion criteria: stroke diagnosed by self-report questionnaire as stroke, subarachnoid haemorrhage & $\begin{array}{l}\text { or transient ischaemic attack. Self-reported clinically significant urinary symptoms, including leakage } \\
\text { of urine, stress incontinence, frequency, nocturia or urgency. }\end{array}$ \\
& $\begin{array}{l}\text { Exclusion criteria: pregnancy, physical causes of urinary tract dysfunction (prolapse, urethral stricture, } \\
\text { prostatic obstruction, pelvic mass or malignancy in past } 5 \text { years), active treatment for incontinence in } \\
\text { past } 6 \text { months, neurogenic retention, raised postresidual volumes, glycosuria or haematuria }\end{array}$
\end{tabular}

Interventions Intervention group: 152 participants. CNP assessment and treatment. CNP received 3 months' training and used formatted assessment and guidelines for treatment, which could include habit retraining, pelvic floor awareness, dietary advice, provision of continence garments and GP referral for treatment of atrophic vaginitis, candidiasis or constipation. Intervention comprised 6 contacts and 5 treatment visits at weeks 1, 2, 4, 6 and 8. Week 1: history + physical examination; week 2: diagnostic visit + development of management plan; weeks 4 and 6: review, adapt + reinforce management plan; week 8: reassessment.

Control group: 80 participants. Usual care provided by GP and referral to existing services for the management of continence.

Outcomes Outcomes measured at 3 and 6 months. Nurse assessment and self-report by participant: frequency,
nocturia, urgency, UI, daytime leakage, night-time leakage

At 3 months: all of the above and satisfaction with service

Funding source NHS Cardiovascular Disease and Stroke National Research and Development Programme

Declarations of inter-

None reported

est among primary re-

searchers 
Brittain 2000b (Continued)

Notes

Subsample of the Medical Research Council Incontinence Study

1:1 randomisation.

No baseline differences in age and gender between groups

Proportions for which data reported: $82 \%$ at 3 months, $63 \%$ at 6 months

\section{Risk of bias}

\begin{tabular}{|c|c|c|}
\hline Bias & Authors' judgement & Support for judgement \\
\hline $\begin{array}{l}\text { Random sequence genera- } \\
\text { tion (selection bias) }\end{array}$ & Low risk & $\begin{array}{l}\text { Quote: "For each batch of subjects, nine individual lists of allocations, (one for } \\
\text { each of the interviewers who will be recruiting subjects) were generated us- } \\
\text { ing the SAS procedure PROC PLAN. Random permuted blocks of } 10 \ldots \text {... (5 GP, } \\
5 \text { CNP) ... These lists were stored as confidential paper lists, and in a read and } \\
\text { write protected Microsoft ACCESS database." }\end{array}$ \\
\hline
\end{tabular}

\begin{tabular}{|c|c|c|}
\hline $\begin{array}{l}\text { Allocation concealment } \\
\text { (selection bias) }\end{array}$ & Unclear risk & $\begin{array}{l}\text { Quote: "Randomisation envelopes were prepared by clerical staff on the study } \\
\text { team. Envelopes had a trial, batch number, list number and envelope number } \\
\text { printed on the front. Stroke randomization envelopes were white." }\end{array}$ \\
\hline
\end{tabular}

Blinding of participants Low risk

No blinding but outcome unlikely to be influenced by lack of blinding.

and personnel (perfor-

mance bias)

All outcomes

Blinding of outcome as-
sessment (detection bias) $\quad$ Low risk blinding but outcome unlikely to be influenced by lack of blinding.

sessment (detection bias)

All outcomes

Incomplete outcome data High risk

(attrition bias)

Large amount of attrition: 190/232 (81.9\%) remaining at 3 months; $147 / 232$

All outcomes

$(63.4 \%)$ remaining at 6 months. Reasons given but not by group.

Selective reporting (re- Unclear risk No protocol available.

porting bias)

Other bias Low risk No significant differences in age and gender between groups.

\begin{tabular}{ll} 
Chu 1997 & \\
\hline Methods & Sesign: RCT \\
& Setting: China \\
\hline Participants & $32(53 \%)$ male and $28(47 \%)$ female adult ischaemic stroke survivors with UI/frequency. \\
& Participants described as having multifocal cerebral infarction. \\
& Age: men $50-71$ years, women $45-61$ years \\
& Inclusion criteria: not reported \\
& Exclusion criteria: not reported \\
\hline
\end{tabular}


Chu 1997 (Continued)

Interventions
Intervention group: 30 participants. Scalp acupuncture using the "bai hui" channel. Needle in situ for 1-2 days in hot weather, and 3-7 days in cold weather. Needle manipulated every 4 hours. Treatment period: 1-2 weeks.

Control group: 30 participants. Usual care. No scalp acupuncture treatment.

Outcomes Prespecified outcomes and timing not reported. 2-week follow-up reported as intervention group totally regaining "normal urine."

Control group: no results given.

\begin{tabular}{ll}
\hline Funding source & Not reported \\
\hline $\begin{array}{l}\text { Declarations of inter- } \\
\text { est among primary re- }\end{array}$ & None reported \\
searchers & \\
\hline
\end{tabular}

Notes

\section{Risk of bias}

Bias Authors' judgement Support for judgement

Random sequence genera- Unclear risk Randomised into 2 groups; no description given. tion (selection bias)

Allocation concealment Unclear risk Insufficient information available to permit judgement.
(selection bias)

Blinding of participants Unclear risk Insufficient information available to permit judgement.
and personnel (performance bias)

All outcomes

\begin{tabular}{ll}
\hline Blinding of outcome as- \\
sessment (detection bias)
\end{tabular}$\quad$ Unclear risk Insufficient information available to permit judgement.

All outcomes

Incomplete outcome data High risk Outcomes not stated prior to treatment.
(attrition bias)
All outcomes

Selective reporting (re- Unclear risk $\quad$ No protocol available.
porting bias)

Other bias High risk Authors "selected 60 patients" from 120 treated during 1992-1994. Women ap-
peared younger than men but it was not stated whether this was statistically significant. No other participant characteristics reported other than gender and age; unable to assess between-group differences prior to intervention

Group 2: "No scalp acupuncture treatment;" unclear what this group received.

Chu 2011

$\begin{array}{ll}\text { Methods } & \text { Design: RCT } \\ & \text { Study dates: hospital inpatients recruited between May } 2007 \text { and October } 2010\end{array}$


Chu 2011 (Continued)

Setting: China, setting unclear

\begin{tabular}{|c|c|}
\hline \multirow{9}{*}{ Participants } & 111 men and women \\
\hline & Number and \% women: intervention 33 (60\%), control 29 (53\%) \\
\hline & Age (mean): Intervention 66.4 (SD 9.32) years, control 64.5 (SD 8.63) years \\
\hline & Time since stroke (days): intervention 35.1 (SD 25.6), control 35.4 (SD 23.9) \\
\hline & Stroke type: \\
\hline & $\begin{array}{l}\text { Intervention group: cerebral haemorrhage } 16(28.6 \%) \text {; cerebral infarction } 40 \text { ( } 71.4 \%) \text {; anepia, loss of } \\
\text { speech } 6(10.7 \%) \text {; vascular dementia } 5(8.9 \%)\end{array}$ \\
\hline & $\begin{array}{l}\text { Control group: cerebral haemorrhage } 14(25.4 \%) \text {; cerebral infarction } 41 \text { ( } 74.5 \%) \text {; anepia, loss of speech } \\
5(9.1 \%) \text {; vascular dementia } 4(7.3 \%)\end{array}$ \\
\hline & $\begin{array}{l}\text { Inclusion criteria: first or repeated episode of stroke, according to the Fourth National Conference } \\
\text { on cerebrovascular disease in 1995; in line with the above described diagnostic criteria; duration } \leq 6 \\
\text { months; age > } 40 \text { years; no incontinence prior to this episode; consenting informed }\end{array}$ \\
\hline & Exclusion criteria: not reported \\
\hline
\end{tabular}

Interventions

Intervention group: 56 participants. Intervention given in addition to control measures (described below). Electroacupuncture at Shengshu points (bilaterally) and Huiyang points (bilateral), with the needle being rotated every 10 minutes. Treatment given at $1 \mathrm{~Hz}$, and titrated to participant's tolerance of electroacupuncture. Given daily once, 30 minutes' duration. Total of 4 weeks, with 1 day break each week. Caution is paid to fainting (therapy immediately stopped with removal of needles and allowing participant to lie flat with head lowered; hot water or sugar water given). Severe cases given emergency treatment, such as: haematoma, local bleeding. Heat compresses given to help reduce scarring.

Control group: 55 participants. Treated according to clinical condition with standard medical therapy, such as lowering blood pressure with calcium channel antagonists, angiotensin-converting enzyme inhibitors, angiotensin 2 receptor antagonists etc.), improving circulation with $500 \mathrm{mg}$ bolus and continuous venous drip after, functional improving therapy such as scalp acupuncture, body acupuncture (acupuncture with rotation of 100 rounds per $\mathrm{min}$ ), done daily for total of 4 weeks.

Outcomes

Outcomes measured pre- and post-intervention (4 weeks): UI grading score; clinical symptoms score (frequency, urgency, incontinence); urinary infection

\begin{tabular}{ll}
\hline Funding source & Not reported \\
\hline $\begin{array}{l}\text { Declarations of inter- } \\
\text { est among primary re- }\end{array}$ & None reported \\
searchers &
\end{tabular}

\section{Notes}

\section{Risk of bias}

\begin{tabular}{lll}
\hline Bias & Authors' judgement & Support for judgement \\
\hline $\begin{array}{l}\text { Random sequence genera- } \\
\text { tion (selection bias) }\end{array}$ & Low risk & $\begin{array}{l}\text { Randomised numbers in envelopes drawn by participants and then checked } \\
\text { against a random number chart for allocation. }\end{array}$ \\
\hline $\begin{array}{l}\text { Allocation concealment } \\
\text { (selection bias) }\end{array}$ & Unclear risk & Sealed envelopes, which then are checked against random number chart. \\
\hline
\end{tabular}


Chu 2011 (Continued)

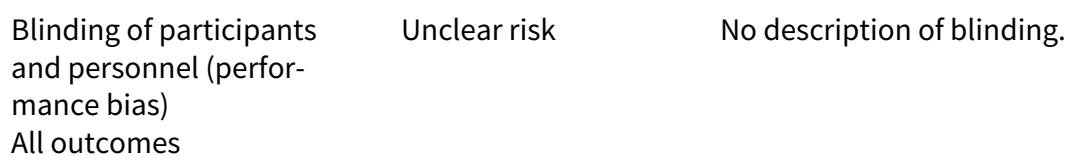

Blinding of outcome as- Unclear risk No description of blinding.
sessment (detection bias)

All outcomes

Incomplete outcome data Low risk Outcomes reported for all participants randomised.

(attrition bias)

All outcomes

\begin{tabular}{lll}
\hline $\begin{array}{l}\text { Selective reporting (re- } \\
\text { porting bias) }\end{array}$ & Unclear risk & No protocol available. \\
\hline Other bias & Low risk & None detected \\
\hline
\end{tabular}

Gelber 1997a

\begin{tabular}{|c|c|}
\hline \multirow[t]{3}{*}{ Methods } & Design: multi-arm RCT (2-arms) \\
\hline & Study dates: not reported \\
\hline & Setting: not reported \\
\hline \multirow[t]{4}{*}{ Participants } & 18 adults \\
\hline & Number and \% women: not reported \\
\hline & Age: not reported \\
\hline & Unilateral stroke survivors with UI and normal urodynamic studies \\
\hline \multirow[t]{3}{*}{ Interventions } & Intervention group: 10 people allocated to void on request \\
\hline & Intervention group: 8 people allocated to timed voiding \\
\hline & No further details given \\
\hline Outcomes & $\begin{array}{l}\text { Number of incontinence episodes per day, averaged for each month of treatment for } 1 \text { year. No numeri- } \\
\text { cal results reported. }\end{array}$ \\
\hline Funding source & Not reported \\
\hline $\begin{array}{l}\text { Declarations of inter- } \\
\text { est among primary re- } \\
\text { searchers }\end{array}$ & None reported \\
\hline \multirow[t]{2}{*}{ Notes } & $\begin{array}{l}\text { Gelber } 1997 \text { comprised of } 4 \text { arms (Gelber } 1997 \mathrm{a} \text { ( } 2 \text { arms) and Gelber } 1997 \mathrm{~b}(2 \text { arms)) with } 37 \text { partici- } \\
\text { pants in total. Randomisation was separate and in } 2 \text { different groups; therefore we reported it as } 2 \times 2- \\
\text { armed trials. }\end{array}$ \\
\hline & This trial was reported as ongoing in a conference abstract. \\
\hline
\end{tabular}

\section{Risk of bias}


Gelber 1997a (Continued)

\section{Bias Authors' judgement Support for judgement}

Random sequence genera- Unclear risk Insufficient evidence about sequence generation process. tion (selection bias)

Allocation concealment $\quad$ Unclear risk $\quad$ Method of concealment not described.
(selection bias)

Blinding of participants Unclear risk Insufficient information available to permit judgement.
and personnel (performance bias)

All outcomes

Blinding of outcome as-
sessment (detection bias) $\quad$ Unclear risk Insufficient information available to permit judgement.

All outcomes

Incomplete outcome data Unclear risk $\quad$ Attrition not reported.
(attrition bias)

All outcomes

Selective reporting (re- Unclear risk No protocol available.
porting bias)

\begin{tabular}{ll}
\hline Other bias $\quad$ Unclear risk $\quad$ Insufficient information to assess whether an important risk of bias exists. \\
\hline
\end{tabular}

Gelber 1997b

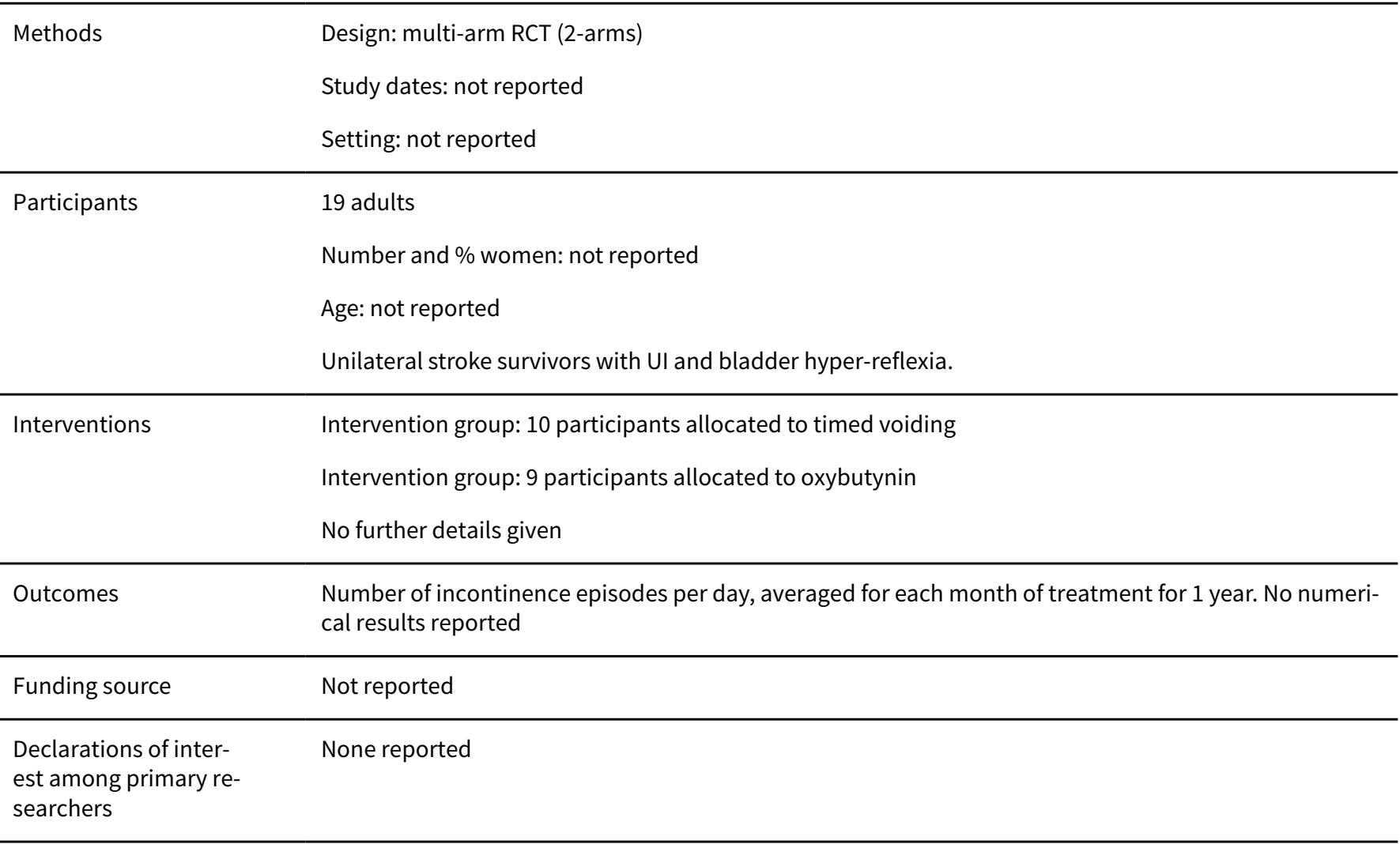


Gelber 1997b (Continued)

Notes
Gelber 1997 comprises of 4 arms (Gelber 1997a ( 2 arms) and Gelber 1997b (2 arms)) with 37 participants in total. Randomisation was separate and in 2 different groups; therefore we reported it as $2 \times 2$ armed trials.

This trial was reported as ongoing in a conference abstract.

\title{
Risk of bias
}

\section{Bias}

Authors' judgement Support for judgement
Random sequence genera- Unclear risk tion (selection bias)

Allocation concealment $\quad$ Unclear risk $\quad$ Method of concealment not described.
(selection bias)

\begin{tabular}{|c|c|c|}
\hline $\begin{array}{l}\text { Blinding of participants } \\
\text { and personnel (perfor- } \\
\text { mance bias) } \\
\text { All outcomes }\end{array}$ & Unclear risk & Insufficient information available to permit judgement. \\
\hline $\begin{array}{l}\text { Blinding of outcome as- } \\
\text { sessment (detection bias) } \\
\text { All outcomes }\end{array}$ & Unclear risk & Insufficient information available to permit judgement. \\
\hline $\begin{array}{l}\text { Incomplete outcome data } \\
\text { (attrition bias) } \\
\text { All outcomes }\end{array}$ & Unclear risk & Attrition not reported. \\
\hline $\begin{array}{l}\text { Selective reporting (re- } \\
\text { porting bias) }\end{array}$ & Unclear risk & No protocol available. \\
\hline Other bias & Unclear risk & Insufficient information to assess whether an important risk of bias exists. \\
\hline
\end{tabular}

Guo 2014

\begin{tabular}{ll}
\hline Methods & Design: RCT \\
Study dates: not reported; participants were hospitalised between January 2010 and January 2011 \\
Setting: China, hospital neurology department
\end{tabular}

\section{Participants}

\author{
61 men and women \\ Number and \% women: 19 (31.2\%) \\ Age: intervention 68.1 (SD 7.1) years, control 65.1 (SD 9.8) years \\ Description of stroke and UI: \\ Ischaemic stroke: intervention 24 participants, control 23 participants \\ Haemorrhagic stroke: intervention 8 participants, control 6 participants \\ Time since stroke: intervention 48.7 (SD 10.5) days, control 53.1 (SD 18.2) days \\ Inclusion criteria: people with post-stroke UI, stable, co-operative and could effectively communicate \\ (to accomplish therapy), and could finish the survey on urination and pre- and post-treatment.
}


Guo 2014 (Continued)

Exclusion criteria: people with urinary retention, various reasons for $\mathrm{Ul}$ in the past (Parkinson's disease, spinal cord disease, dementia, urinary tract tumours, urolithiasis, unhealed UTIs or history of urinary tract surgery), severe cognitive function disorders (Mini-Mental State Examination 14-22 points), unable to communicate effectively, concurrent serious organic diseases, needed massive fluid infusion or usage of drugs that affected urination and unable to provide accurate survey information or to complete the treatment period.

Interventions

Intervention group: 32 participants. TENS (model TENS-21; Homer Ion Laboratory Company Ltd, Tokyo, Japan) for 30 minutes once per day for 60 days with pulse duration $70 \mu \mathrm{S}$ and frequency $75 \mathrm{~Hz}$ in form of unidirectional square wave. Maximum therapeutic current $16 \mathrm{~mA}(1 \mathrm{k} \Omega)$. According to the nerve innervation, the electrodes' pads were placed as follows: the positive electrode $\left(39 \mathrm{~cm}^{2}\right)$ was on the second lumbar spinous process and 2 negative electrodes $\left(30 \mathrm{~cm}^{2}\right)$ were on the inside of the middle and lower third of the junction between the posterior superior iliac spine and the ischia node. Reusable silicone gel electrodes were used in the study and a small amount of water was placed in the electrodes to make good electrical contact.

Control group: 29 participants. Basic therapy.

Outcomes Measured pre- and post-intervention (60 days)

OABSS (self-report), Barthel Index, urodynamic examination (daily micturitions, nocturia, urgency, UI)

Funding source Not reported

Declarations of inter- Author reported no conflict of interests.

est among primary re-

searchers

Notes

\section{Risk of bias}

\begin{tabular}{lll}
\hline Bias & Authors' judgement & Support for judgement \\
\hline $\begin{array}{l}\text { Random sequence genera- } \\
\text { tion (selection bias) }\end{array}$ & Unclear risk & Participants randomly divided into 2 groups. \\
\hline $\begin{array}{l}\text { Allocation concealment } \\
\text { (selection bias) }\end{array}$ & Unclear risk & Not described \\
\hline $\begin{array}{l}\text { Blinding of participants } \\
\text { and personnel (perfor- } \\
\text { mance bias) } \\
\text { All outcomes }\end{array}$ & Unclear risk & Insufficient information to permit judgement of low or high risk. \\
\hline
\end{tabular}

Blinding of outcome as- Unclear risk Insufficient information to permit judgement of low or high risk.

sessment (detection bias)

All outcomes

Incomplete outcome data Unclear risk (attrition bias)

All outcomes
Outcome data in Table 2 completed for all participants. For Table 3 and Table 4 it was not clear if any participants missing from analyses. For Table 3 , the Barthel Index score was $>50$ points for $21(65.63 \%)$ participants in the treatment group but for only $10(34.48 \%)$ participants in the control group (Table 2); there were no further details on other participants. For Table 4, there were no details of numbers of participants in analyses in either group or text.

\begin{tabular}{l}
$\begin{array}{l}\text { Selective reporting (re- Unclear risk } \\
\text { porting bias) }\end{array}$ \\
\hline
\end{tabular}


Guo 2014 (Continued)

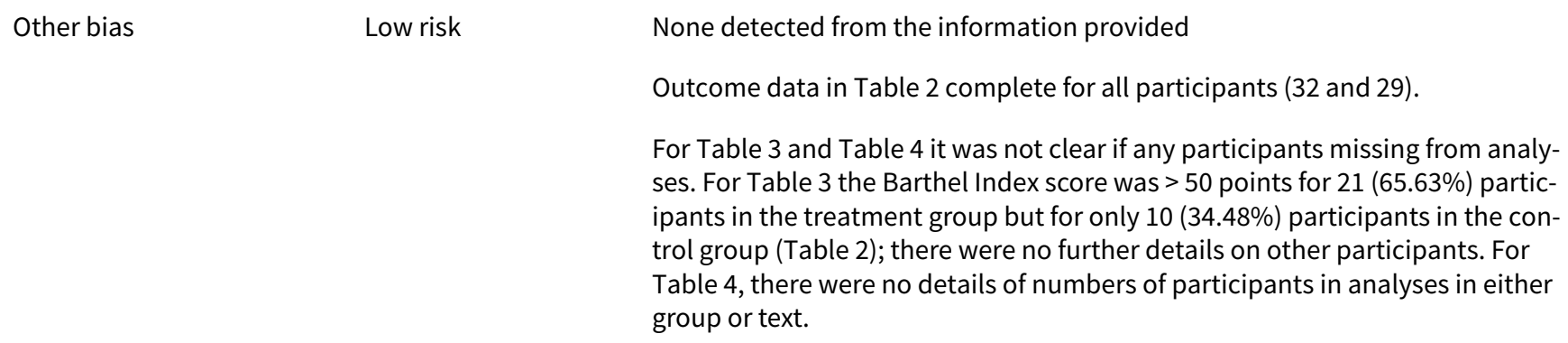

Judge 1969

\begin{tabular}{|c|c|}
\hline Methods & $\begin{array}{l}\text { Design: double-blind cross-over trial } \\
\text { Study dates: not reported } \\
\text { Setting: UK, Scotland, long-stay geriatric hospitals }\end{array}$ \\
\hline Participants & $\begin{array}{l}12 \text { women (stroke subgroup) } \\
\text { Age: mean } 82 \text { years, range } 66-92 \text { years } \\
\text { 12/18 women with diagnosis of cerebrovascular accident or "multiple little strokes", } 7 \text { with mild incon- } \\
\text { tinence (mean } 12.3 \text { episodes per week), and } 5 \text { with severe incontinence (mean } 41.1 \text { episodes per week). } \\
\text { Inclusion criteria: none stated } \\
\text { Exclusion criteria: faecal impaction, urinary infection }\end{array}$ \\
\hline
\end{tabular}

Interventions

Intervention group: quinestradol $0.25 \mathrm{mg} 4$ times per day for 1 month

Control group: placebo with a washout period of 1 month

\begin{tabular}{|c|c|}
\hline Outcomes & Baseline number of incontinent episodes per week and number of incontinent episodes at week 5 \\
\hline Funding source & William R. Warner and Co supplied the Pentovis for the trial \\
\hline $\begin{array}{l}\text { Declarations of inter- } \\
\text { est among primary re- } \\
\text { searchers }\end{array}$ & None reported \\
\hline \multirow[t]{3}{*}{ Notes } & $\begin{array}{l}\text { Participants in intervention group (defined as 'mildly incontinent') and control group (defined as 'se- } \\
\text { verely incontinent') were from } 2 \text { different hospitals. }\end{array}$ \\
\hline & $\begin{array}{l}\text { Most participants were mentally confused, } 8 \text { participants were classified as 'chairfast' and } 4 \text { partici- } \\
\text { pants were classified as 'bedfast.' }\end{array}$ \\
\hline & $\begin{array}{l}20 \text { participants recruited with data reported for } 18 / 20 \text { participants, } 1 \text { participant died, } 1 \text { withdrew. The } \\
\text { stroke subgroup consisted of } 12 \text { participants. }\end{array}$ \\
\hline
\end{tabular}

\section{Risk of bias}

\begin{tabular}{lll}
\hline Bias & Authors' judgement & Support for judgement \\
\hline $\begin{array}{l}\text { Random sequence genera- } \\
\text { tion (selection bias) }\end{array}$ & Low risk & Quote: "A set of random numbers was used." \\
\hline $\begin{array}{l}\text { Allocation concealment } \\
\text { (selection bias) }\end{array}$ & Low risk & $\begin{array}{l}\text { Quote: "The hospital Pharmacist was provided with active product and place- } \\
\text { bo labelled only 'Capsules } X \text { ' and 'Capsules } Y \text { ' along with a sealed key for emer- }\end{array}$ \\
\hline
\end{tabular}

Interventions for treating urinary incontinence after stroke in adults (Review)

Copyright $\odot 2019$ The Cochrane Collaboration. Published by John Wiley \& Sons, Ltd. 
Comment: pharmacy controlled randomisation.

\begin{tabular}{|c|c|c|}
\hline $\begin{array}{l}\text { Blinding of participants } \\
\text { and personnel (perfor- } \\
\text { mance bias) } \\
\text { All outcomes }\end{array}$ & Low risk & Quote: "A strict double-blind crossover technique was used." \\
\hline $\begin{array}{l}\text { Blinding of outcome as- } \\
\text { sessment (detection bias) } \\
\text { All outcomes }\end{array}$ & Unclear risk & Insufficient information available to permit judgement. \\
\hline $\begin{array}{l}\text { Incomplete outcome data } \\
\text { (attrition bias) } \\
\text { All outcomes }\end{array}$ & Low risk & $\begin{array}{l}\text { Data available for all but } 2 \text { participants. } 1 \text { participant died in the early stages of } \\
\text { the trial, } 1 \text { became too confused to take the capsules following a second cere- } \\
\text { brovascular accident. }\end{array}$ \\
\hline $\begin{array}{l}\text { Selective reporting (re- } \\
\text { porting bias) }\end{array}$ & Unclear risk & No protocol available. \\
\hline Other bias & Unclear risk & Most participants were mentally confused \\
\hline
\end{tabular}

Lewis 1990

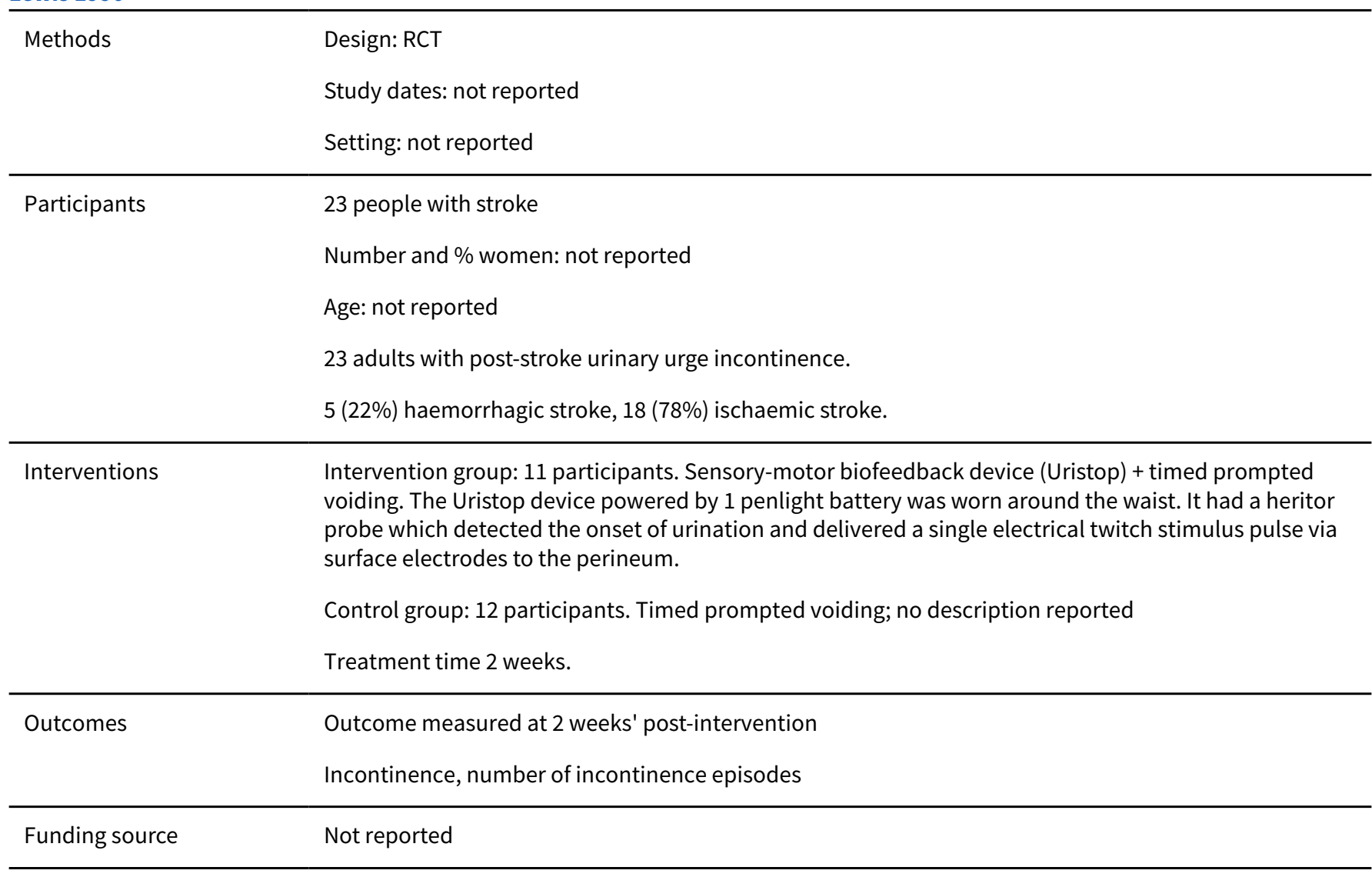


Lewis 1990 (Continued)

Declarations of interest among primary re-

None reported

searchers

Notes Continence defined as 0 incontinent episodes in the last 2 days of study.

Trial reported in a conference abstract.

\section{Risk of bias}

\begin{tabular}{lll}
\hline Bias & Authors' judgement & Support for judgement \\
\hline $\begin{array}{l}\text { Random sequence genera- } \\
\text { tion (selection bias) }\end{array}$ & Unclear risk & Insufficient evidence about the sequence generation process. \\
\hline $\begin{array}{l}\text { Allocation concealment } \\
\text { (selection bias) }\end{array}$ & Unclear risk & Method of concealment not described. \\
\hline $\begin{array}{l}\text { Blinding of participants } \\
\text { and personnel (perfor- } \\
\text { mance bias) } \\
\text { All outcomes }\end{array}$ & Unclear risk & Insufficient information available to permit judgement. \\
\hline
\end{tabular}

\begin{tabular}{lll}
\hline $\begin{array}{l}\text { Blinding of outcome as- } \\
\text { sessment (detection bias) } \\
\text { All outcomes }\end{array}$ & Unclear risk & Insufficient information available to permit judgement. \\
\hline $\begin{array}{l}\text { Incomplete outcome data } \\
\text { (attrition bias) } \\
\text { All outcomes }\end{array}$ & Unclear risk & Attrition not reported. \\
\hline $\begin{array}{l}\text { Selective reporting (re- } \\
\text { porting bias) }\end{array}$ & Unclear risk & No protocol available. \\
\hline Other bias & Unclear risk & Insufficient information to assess whether an important risk of bias existed. \\
\hline
\end{tabular}

\section{Liu 2006}

\begin{tabular}{ll}
\hline Methods & Design: RCT \\
& Study dates: October 2004 to September 2006 \\
& Setting: China, inpatient \\
\hline
\end{tabular}

35 men (47\%) and 40 women (53\%) stroke survivors with UI.

Number of women in each arm: intervention 18 (46.1\%), control 17 (47.2\%)

Age (mean): intervention 64.7 (SD 7.48) years, control 62.3 (SD 8.41) years

Cerebral infarction: intervention 30 (76,9\%), control 30 (83.3\%)

Cerebral haemorrhage: intervention 9 (23.01\%), control $6(16.7 \%)$

Inclusion criteria: cerebral infarction or haemorrhage, time between stroke onset and admission to hospital $<6$ months, continent of urine prestroke, presence of UI, urgent or frequent micturition when condition stabilised after stroke, micturition problems affected quality of life, conscious and able to communicate, normal recognition, aged $40-75$ years. 
Liu 2006 (Continued)

Exclusion criteria: comorbidities involving the heart, kidney or other important organs; prestroke chronic urinary retention and UI, unable to communicate, long-standing chronic UTI.

Interventions

Intervention group: 39 participants. Ginger-salt-partitioned moxibustion at Sheque (CV 8) and routine acupuncture (e.g. Tsusanli, Yinlingquan). Treatments delivered 5 times per week for 3 weeks.

Control group: 36 participants. Routine acupuncture

\begin{tabular}{ll}
\hline Outcomes & $\begin{array}{l}\text { Outcome measures reported before and after treatment at } 3 \text { weeks } \\
\text { Mean number of urination times each day, mean number of times requesting toileting at night, number } \\
\text { of participants with incontinence at } 72 \text { hours, urgent Ul during day }\end{array}$ \\
\hline $\begin{array}{l}\text { Funding source } \\
\begin{array}{l}\text { Declarations of inter- } \\
\text { est among primary re- } \\
\text { searchers }\end{array}\end{array}$ & None reported in translation. \\
\hline Notes & Study reported in Chinese and translated into English. \\
& $\begin{array}{l}\text { No baseline differences in age, gender, type of stroke, stroke course, frequency symptoms and grade of } \\
\text { Ul between the groups. }\end{array}$ \\
\hline
\end{tabular}

\section{Risk of bias}

\begin{tabular}{|c|c|c|}
\hline Bias & Authors' judgement & Support for judgement \\
\hline $\begin{array}{l}\text { Random sequence genera- } \\
\text { tion (selection bias) }\end{array}$ & Unclear risk & Abstract stated "randomly divided;" no further information provided. \\
\hline $\begin{array}{l}\text { Allocation concealment } \\
\text { (selection bias) }\end{array}$ & Unclear risk & Not described \\
\hline $\begin{array}{l}\text { Blinding of participants } \\
\text { and personnel (perfor- } \\
\text { mance bias) } \\
\text { All outcomes }\end{array}$ & Unclear risk & Not described \\
\hline $\begin{array}{l}\text { Blinding of outcome as- } \\
\text { sessment (detection bias) } \\
\text { All outcomes }\end{array}$ & Unclear risk & Not described \\
\hline $\begin{array}{l}\text { Incomplete outcome data } \\
\text { (attrition bias) } \\
\text { All outcomes }\end{array}$ & Low risk & $\begin{array}{l}\text { Appeared complete; data analysis excluded participants with catheters. Attri- } \\
\text { tion reasons reported for both groups. }\end{array}$ \\
\hline $\begin{array}{l}\text { Selective reporting (re- } \\
\text { porting bias) }\end{array}$ & Unclear risk & Protocol not available. \\
\hline Other bias & Low risk & None detected \\
\hline
\end{tabular}

Liu 2013

$\begin{array}{ll}\text { Methods } & \text { Design: randomised, double-blind, placebo-controlled } \\ & \text { Study dates: June } 2008 \text { to June } 2012\end{array}$


Liu 2013 (Continued)

Setting: China, outpatient, Department of Neurology

Participants

71 men and women

Number and \% women: intervention 12 (36.4\%), control $13(39.4 \%)$

Age (mean): intervention 39 (SD 12) years, control 37 (SD 11) years

Description of stroke and UI: all participants had a history of cerebral infarction or cerebral haemorrhage but the sites of the lesion were different in the 2 groups and the numbers of participants with lesions at specific sites were small. Acute stroke diagnosed by a physician and cerebral haemorrhage or infarction observed on cranial CT or MRI.

Infarction: intervention 13 (39.4\%), control 17 (51.5\%)

Haemorrhagic: intervention 20 (60.6\%), control 16 (48.5\%)

Detrusor overactivity assessed using voiding diaries and urodynamic testing according to the AUA/SUFA guidelines using a urodynamic testing system (Laborie Medical Technologies, Beijing, China).

Inclusion criteria: aged 20-50 years, acute stroke diagnosed by a physician and cerebral haemorrhage or infarction observed on cranial CT or MRI; stable condition, and mobile in a wheelchair or undergoing rehabilitation, for $\geq 6$ months; no indwelling urinary catheter.

Exclusion criteria: previous acupuncture treatment; pregnant or planning pregnancy; implanted pacemaker; implanted neurostimulator; cardiac arrhythmia; epilepsy; severe heart, brain, lung or other major organ disease; acid-base or electrolyte imbalance; history of severe kidney disease, hydronephrosis, bladder stoma, sphincterotomy or severe exaggeration of autonomic nervous system reflexes; UTI; cognitive disorder and acupuncture syncope.

Interventions

Intervention group: 35 participants. Electroacupuncture. Considering the differences among participants, individualised acupuncture administered in combination with traditional Chinese acupuncture methods. According to modern acupuncture methods, points Baliao (including bilateral Shangliao (BL31), bilateral Ciliao (BL32), bilateral Zhongliao (BL33), and bilateral Xialiao (BL34)) and Huiyang, which are closely associated with the sacral micturition centre, were selected. Participants placed in lateral or prone position. Entire procedure performed under sterile conditions. Target skin sterilised using 75\% ethanol and stainless steel 30-gauge acupuncture needles (Guizhou Andi Medicated Instruments, Guizhou, China) inserted medially and obliquely into points Baliao and Huiyang. Needles inserted to a depth of $3.5-4.0 \mathrm{~cm}$ at points Baliao, at the first to fourth dorsal sacral foramina, and to a depth of 3.5-5.0 cm at point Huiyang. De qi (a sensation of numbness and distension at the needling site) was achieved by manual stimulation. Electroacupuncture $(30-40 \mathrm{~Hz}$, continuous wave) performed at points Xialiao and Huiyang. Electroacupuncture then performed at the remaining points using the HM6805-2 Acupoint Pulse Therapy Instrument (20-30 mA; Sichuan Hengming Technology Development, Sichuan, China). Stimulation intensity adjusted to achieve rhythmic muscle contractions in anus and perineal region and be tolerable to the participants. After completion of acupuncture, the acupuncturist left and the participant rested on bed. Electroacupuncture performed daily for 10 consecutive days, and needles inserted for 20 minutes each time. Only 1 set of interventions administered.

Control group: 36 participants. Sham electroacupuncture, as recommended by WHO. Points Baliao were used, which are closely associated with the sacral micturition centre. Needles attached to $1 \mathrm{~cm}$ diameter rings, which were attached to participant using surgical tape. Positions of the participant and acupuncturist, procedures, duration of stimulation, and timing of interventions identical to procedures in electroacupuncture group. Needles attached to positions $1 \mathrm{~cm}$ lateral to the acupoints. Sham acupuncture needles did not pierce the skin. The electrical wire used for sham electroacupuncture was broken so it could not conduct electricity. After the intervention, the acupuncturist left and the participant rested on the bed. The needles were left in position for 20 minutes each time. Sham electroacupuncture was performed daily for 10 consecutive days.

Outcomes

Outcomes measured at the beginning and end of treatment and at the 3 month follow-up: mean maximum cystometric capacity, bladder compliance, detrusor leak point pressure, frequency of urination and UI. 
Liu 2013 (Continued)

Funding source Study supported by the Postdoctoral Foundation of Heilongjiang Province, No. LRB2008-384

Declarations of inter- Authors declared no conflicts of interests.

est among primary re-

searchers

Notes

\section{Risk of bias}

Bias Authors' judgement Support for judgement

Random sequence genera- Unclear risk Quote: "The subjects were randomly assigned."

tion (selection bias)

Allocation concealment Unclear risk Quote: "To ensure that the proportions of patients with severe urinary fre(selection bias) quency and incontinence were similar in both groups, these parameters were taken into consideration during group assignment. The group of each subject was recorded, and the record was placed in an opaque envelope and sent to the investigator. The investigator opened the envelope and informed the acupuncturist of the group assignment."

Comment: group selection appeared to take place after randomisation. Unclear if envelopes were sequentially numbered and sealed.

\begin{tabular}{|c|c|c|}
\hline $\begin{array}{l}\text { Blinding of participants } \\
\text { and personnel (perfor- } \\
\text { mance bias) } \\
\text { All outcomes }\end{array}$ & Low risk & Quote: "The subjects and evaluators were blinded to the group assignment." \\
\hline $\begin{array}{l}\text { Blinding of outcome as- } \\
\text { sessment (detection bias) } \\
\text { All outcomes }\end{array}$ & Low risk & Quote: "The subjects and evaluators were blinded to the group assignment." \\
\hline $\begin{array}{l}\text { Incomplete outcome data } \\
\text { (attrition bias) } \\
\text { All outcomes }\end{array}$ & Unclear risk & $\begin{array}{l}\text { Numbers of participants included in analysis not reported. } 2 / 35 \text { dropped out } \\
\text { of intervention group, } 3 / 36 \text { dropped out of control group with reasons provid- } \\
\text { ed. }\end{array}$ \\
\hline $\begin{array}{l}\text { Selective reporting (re- } \\
\text { porting bias) }\end{array}$ & Unclear risk & No protocol available. \\
\hline Other bias & Low risk & None detected. \\
\hline
\end{tabular}

Liu 2016a

Methods Design: multi-arm RCT (3-arm)

Study dates: participants recruited during hospitalisation between July 2011 and January 2013

Setting: China, inpatients or outpatients (group A and B), outpatient (group C)

Participants
Number and \% women: group A 5 (18.52\%), group B 8 (29.63\%), group C 10 (37.04\%)
Age (mean): group A 66.30 (10.84) years, group B 63.75 (8.92) years, group C 67.91 (7.39) years


Liu 2016a (Continued)

Description of stroke and UI:

Cerebral haemorrhage: group A 6 (22.22\%), group B 9 (33.33), group C 7 (25.93)

Cerebral infarction: group A 21 (77.78), group B 18 (66.67), group C 20 (74.07)

Time since stroke (mean): group A 65.96 (SD 9.39) days, group B 71.01 (SD 14.86) days, group C 67.82 (SD 11.58) days

Inclusion criteria: people with UI after stroke who were stable, co-operative and could effectively communicate to participate in therapy; and people who could complete the survey on urination both before and after treatment.

Exclusion criteria: people with urinary retention, history of $\mathrm{UI}$ for various reasons (Parkinson's disease, spinal cord disease, dementia, urinary tract tumours, urolithias, unhealed UTIs or history of urinary tract surgery); needed significant fluid infusions or drugs that affected urination; concurrent serious organic diseases; severe cognitive functioning disorders (Mini-Mental State Examination score $<22$ points); unable to communicate effectively; unable to provide accurate survey information or to complete the treatment period.

Interventions

Groups A and B (interventions): 27 participants in each group. TENS (model EN-Stim 4; ENRAF-NONIUS Company Ltd. Rotterdam, the Netherlands) for 30 minutes once daily for 90 days in hospital (as inpatients or outpatients). The TENS device used a 4-channel stimulator with fully independent channels and 4 sets of moist pads for rubber electrodes $(6 \times 8 \mathrm{~cm})$. This process makes it possible to treat major muscle groups simultaneously or to perform bilateral treatments simultaneously. The TENS currents were biphasic square waves with pulse durations of $150 \mu$ seconds and pulse frequencies of $20 \mathrm{~Hz}$ (group A) or $75 \mathrm{~Hz}$ (group B). Underwent OABSS, Barthel Index, urodynamics and voiding diary assessments on the first day and at 90 days.

Group C (control): 27 participants. OABSS, Barthel Index, urodynamics and voiding diary assessments on the first day and at 90 days.

Outcomes

Outcomes measured at day 1 (baseline) and day 90 (last day of the intervention).

OABSS, Barthel Index, voiding diary, urodynamic values

Funding source

Declarations of inter-

est among primary re-

searchers
No funding grants or equipment provided from any source. No financial benefits to authors.

Authors declared no conflicts of interest.

Notes Liu 2016a and Liu 2016b are two arms of a multi-arm RCT reporting on two separate comparisons.

Liu 2016a referred to group A $(20 \mathrm{~Hz})$ and group C (control)

\section{Risk of bias}

\begin{tabular}{lll}
\hline Bias & Authors' judgement & Support for judgement \\
\hline $\begin{array}{l}\text { Random sequence genera- } \\
\text { tion (selection bias) }\end{array}$ & Low risk & Quote: "Randomization was computer generated." \\
\hline $\begin{array}{l}\text { Allocation concealment } \\
\text { (selection bias) }\end{array}$ & Low risk & Quote: "the allocation was centrally generated and concealed." \\
\hline $\begin{array}{l}\text { Blinding of participants } \\
\text { and personnel (perfor- } \\
\text { mance bias) }\end{array}$ & Low risk & \\
$\begin{array}{l}\text { All outcomes } \\
\text { Q }\end{array}$ & $\begin{array}{l}\text { Quote: "both the patients and therapists were blinded to the treatment group- } \\
\text { S" (group A and group C). }\end{array}$ \\
\end{tabular}


Liu 2016a (Continued)

Blinding of outcome as- Low risk Quote: "An independent physician who did not participate in the clinical mansessment (detection bias)

All outcomes agement and who was kept blinded to treatment allocation conducted the assessment."

Incomplete outcome data Low risk No participants dropped out of study. Complete outcome data.
(attrition bias)

All outcomes

Selective reporting (re- Unclear risk $\quad$ No protocol available.
porting bias)

Other bias Low risk None detected.

\section{Liu 2016b}

$\begin{array}{ll}\text { Methods } & \text { Design: multi-arm RCT (3-arm) } \\ \text { Study dates: participants recruited during hospitalisation between July } 2011 \text { and January } 2013 \\ \text { Setting: China, inpatients or outpatients (group A and B), outpatient (group C) }\end{array}$

Participants 81 men and women

Number and \% women: group A 5 (18.52\%), group B 8 (29.63\%), group C 10 (37.04\%)

Age (mean): group A 66.30 (10.84) years, group B 63.75 (8.92) years, group C 67.91 (7.39) years

Description of stroke and UI:

Cerebral haemorrhage: group A 6 (22.22\%), group B 9 (33.33), group C 7 (25.93)

Cerebral infarction: group A 21 (77.78), group B 18 (66.67), group C 20 (74.07)

Time since stroke (mean): group A 65.96 (SD 9.39) days, group B 71.01 (SD 14.86) days, group C 67.82 (SD 11.58) days

Inclusion criteria: people with UI after stroke who were stable, co-operative and could effectively communicate to participate in therapy; and people who could complete the survey on urination both before and after treatment.

Exclusion criteria: people with urinary retention, history of $\mathrm{UI}$ for various reasons (Parkinson's disease, spinal cord disease, dementia, urinary tract tumours, urolithias, unhealed UTIs or history of urinary tract surgery); needed significant fluid infusions or drugs that affected urination; concurrent serious organic diseases; severe cognitive functioning disorders (Mini-Mental State Examination score $<22$ points); unable to communicate effectively; unable to provide accurate survey information or to complete the treatment period.

Interventions

Groups A and B (interventions): 27 participants. TENS (model EN-Stim 4; ENRAF-NONIUS Company Ltd. Rotterdam, the Netherlands) for 30 minutes once daily for 90 days in hospital (as inpatients or outpatients). The TENS device used a 4-channel stimulator with fully independent channels and 4 sets of moist pads for rubber electrodes $(6 \times 8 \mathrm{~cm})$. This process makes it possible to treat major muscle groups simultaneously or to perform bilateral treatments simultaneously. The TENS currents were biphasic square waves with pulse durations of $150 \mu$ seconds and pulse frequencies of $20 \mathrm{~Hz}$ (group A) or $75 \mathrm{~Hz}$ (group B). Underwent OABSS, Barthel Index, urodynamics and voiding diary assessments on the first day and at 90 days.

Group C (control): 27 participants. OABSS, Barthel Index, urodynamics and voiding diary assessments on the first day and at 90 days. 
Liu 2016b (Continued)

Outcomes Outcomes measured at day 1 (baseline) and day 90 (last day of the intervention).

OABSS, Barthel Index, voiding diary, urodynamic values

Funding source

No funding grants or equipment provided from any source. No financial benefits to authors.

Declarations of inter-

est among primary re-

searchers

Authors declared no conflicts of interest.

Notes Liu 2016a and Liu 2016b are two arms of a multi-arm RCT reporting on two separate comparisons.

Liu 2016b referred to group B (75 Hz) and group C (control)

\section{Risk of bias}

\begin{tabular}{|c|c|c|}
\hline Bias & Authors' judgement & Support for judgement \\
\hline $\begin{array}{l}\text { Random sequence genera- } \\
\text { tion (selection bias) }\end{array}$ & Low risk & Quote: "Randomization was computer generated." \\
\hline $\begin{array}{l}\text { Allocation concealment } \\
\text { (selection bias) }\end{array}$ & Low risk & Quote: "the allocation was centrally generated and concealed." \\
\hline $\begin{array}{l}\text { Blinding of participants } \\
\text { and personnel (perfor- } \\
\text { mance bias) }\end{array}$ & Low risk & $\begin{array}{l}\text { Quote: "both the patients and therapists were blinded to the treatment group- } \\
\text { s" (group A and group B). }\end{array}$ \\
\hline All outcomes & & $\begin{array}{l}\text { Comment: neither participants nor therapists blinded to allocation to no treat- } \\
\text { ment/treatment but unlikely outcome influenced by lack of blinding. }\end{array}$ \\
\hline $\begin{array}{l}\text { Blinding of outcome as- } \\
\text { sessment (detection bias) } \\
\text { All outcomes }\end{array}$ & Low risk & $\begin{array}{l}\text { Quote: "An independent physician who did not participate in the clinical man- } \\
\text { agement and who was kept blinded to treatment allocation conducted the as- } \\
\text { sessment." }\end{array}$ \\
\hline $\begin{array}{l}\text { Incomplete outcome data } \\
\text { (attrition bias) } \\
\text { All outcomes }\end{array}$ & Low risk & No participants dropped out of study. Complete outcome data. \\
\hline $\begin{array}{l}\text { Selective reporting (re- } \\
\text { porting bias) }\end{array}$ & Unclear risk & No protocol available. \\
\hline Other bias & Low risk & None detected. \\
\hline
\end{tabular}

Monteiro 2014

\begin{tabular}{ll}
\hline Methods & Design: RCT \\
& Study dates: February 2007 to June 2008 \\
& Setting: Brazil, stroke outpatient \\
\hline Participants & 24 men \\
& Age (mean): intervention 65.1 (SD 3.6) years, control 56.1 (SD 10.9) years \\
& Description of stroke and UI: \\
& Right hemisphere stroke: intervention 7 (58.3\%), control 8 (67\%)
\end{tabular}


Inclusion criteria: men who had stroke aged $\geq 18$ years, stroke occurring 6 months to 3 years before recruitment

Exclusion criteria: men who had implanted cardiac pacemaker, UTI, bladder cancer, pre-existing UI before stroke, surgery in urogenital region

\begin{abstract}
Interventions Intervention group: 12 participants. Electrical stimulation with the Duplex-961 system (Quark, Piracicaba, São Paulo, Brazil). With the participant seated, surface electrodes placed on skin with conductive gel and secured with adhesive tape. A negative electrode placed on medial malleolus and positive electrode placed $10 \mathrm{~cm}$ above the negative electrode, also on the medial side. Rhythmic flexion of second toe during stimulation determined the correct position of the negative electrode. Intensity level set below threshold that caused motor contraction because participant should have been comfortable and with no pain during procedure. Electrical stimulation of the posterior tibialis nerve performed for 30 minutes twice weekly over 12 sessions (45 days), with frequency of $10 \mathrm{~Hz}$ and pulse width of $200 \mathrm{msec}-$ onds in continuous mode.
\end{abstract}

Control group: 12 participants. 12 stretching sessions of lower limbs. 3 muscle-stretching training sessions conducted to instruct participants how to perform each of these 3 series of 30-second stretching exercises at home. Participants did not receive other types of physical therapy or any specific behavioural guidelines for overactive bladder during 12-month follow-up.

\title{
Outcomes
} Outcomes measured before treatment, after treatment (12 sessions, 45 days) and 12-month follow-up. 3-day voiding diary (urgency, urge incontinence, nocturia, nocturnal enuresis, increased daytime frequency

Barthel index

At 12 months: self-report of symptom improvement

\begin{tabular}{ll}
\hline Funding source & Not reported \\
\hline $\begin{array}{l}\text { Declarations of inter- } \\
\text { est among primary re- } \\
\text { searchers }\end{array}$ & None stated \\
\hline Notes & $\begin{array}{l}\text { Authors reported significant difference in age between groups, intervention group older than control } \\
\text { group. No other baseline differences. }\end{array}$
\end{tabular}

\section{Risk of bias}

Bias Authors' judgement Support for judgement

Random sequence genera- Unclear risk tion (selection bias)

Quote: "To ensure random allocation, all participants were numbered sequentially from 1 to 24 and divided into 2 groups of 12 patients assigned to the treatment group (electrical stimulation of the posterior tibialis nerve) and 12 to the control group."

Comment: insufficient information to permit judgement.

\begin{tabular}{lll}
\hline $\begin{array}{l}\text { Allocation concealment } \\
\text { (selection bias) }\end{array}$ & Unclear risk & Insufficient information to permit judgement. \\
\hline $\begin{array}{l}\text { Blinding of participants } \\
\text { and personnel (perfor- }\end{array}$ & Unclear risk & Insufficient information to permit judgement. \\
mance bias) & \\
All outcomes & \\
\hline
\end{tabular}


Monteiro 2014 (Continued)

Blinding of outcome as- Unclear risk Insufficient information to permit judgement. sessment (detection bias)

All outcomes

$\begin{array}{ll}\begin{array}{l}\text { Incomplete outcome data } \\ \text { (attrition bias) }\end{array} & \text { Unclear risk } \\ \text { All outcomes } & \text { Only } 1 \text { participant in the control group dropped out due to death. } \\ & \begin{array}{l}\text { Numbers given for each group in the findings table given as } 36 \text { per group. How- } \\ \text { ever, only } 24 \text { participants randomised. }\end{array}\end{array}$

Selective reporting (re- Unclear risk No protocol available.

porting bias)

Other bias

Low risk

Baseline imbalance in age: intervention group older than control group (65.1

(SD 3.6) years vs 56.1 (SD 10.9) years). Authors suggested that if the groups had been equal, the effect of the intervention might have been demonstrated even more clearly.

Shin 2016a

Design: randomised, single-blind controlled study
Study dates: not reported
Setting: Korea, participants recruited from a rehabilitation centre.

Participants 35 women

Age (mean): intervention 62.08 (SD 3.32) years, control 62.92 (SD 4.93) years.

Description of stroke and UI:

Paretic side: intervention: left 6 (37.5\%), right 10 (62.5\%); control: left 7 (46.7\%), right 8 (53.3\%)

Time since stroke: intervention 6.17 (SD 1.53) months, control 5.17 (SD 2.08) months

Inclusion criteria: > 3 months post-stroke, had UI after stroke, experienced normal vaginal delivery, could understand and follow simple verbal instructions (Korean version of Mini-Mental State Examination score) and no other treatments administered for UI.

Exclusion criteria: UTI, symptoms of vaginal prolapse, chronic respiratory diseases, psychiatric diseases, and other neurological diseases.

Interventions

Intervention: 16 participants. General rehabilitation + education of UI and the functions of the PFMs + PFMT. General rehabilitation training consisting of gait training and stretching not affecting the PFMs for 50 minutes, 3 times per week for 6 weeks. In addition: participants received PFMT for 50 minutes, 3 times per week for 6 weeks. PFMT involved the education of $U$ I and the functions of PFMs, recognition training, training in a range of respiratory conditions and PFMs resistance training. Education of $\mathrm{UI}$ and the functions of the PFMs carried out by a trained physiotherapist with specific knowledge on the basic anatomy and PFM function. To improve recognition of the PFMs, a sensory ball with protruding projections was used on the surface provided individually.

Control: 15 participants. General rehabilitation + education of UI and the functions of the PFMs.

Outcomes Outcomes were measured pre- and post-intervention (6 weeks).

Electromyography, Bristol Female Urinary Symptoms Questionnaire 
Shin 2016a (Continued)

Funding source
This research received no specific grant from any funding agency in the public, commercial or not-forprofit sectors.
Declarations of interest among primary researchers
Authors declared no conflicts of interest.

\section{Notes}

\section{Risk of bias}

\begin{tabular}{|c|c|c|}
\hline Bias & Authors' judgement & Support for judgement \\
\hline $\begin{array}{l}\text { Random sequence genera- } \\
\text { tion (selection bias) }\end{array}$ & Low risk & $\begin{array}{l}\text { To minimise selection bias, participants were assigned randomly to either a } \\
\text { PFMT group or control group using Random Allocation Software. }\end{array}$ \\
\hline $\begin{array}{l}\text { Allocation concealment } \\
\text { (selection bias) }\end{array}$ & Unclear risk & Not described. \\
\hline $\begin{array}{l}\text { Blinding of participants } \\
\text { and personnel (perfor- } \\
\text { mance bias) } \\
\text { All outcomes }\end{array}$ & Low risk & Outcomes not likely to be influenced by lack of blinding. \\
\hline $\begin{array}{l}\text { Blinding of outcome as- } \\
\text { sessment (detection bias) } \\
\text { All outcomes }\end{array}$ & Low risk & $\begin{array}{l}2 \text { trained raters tested maximum vaginal squeeze pressure, PFMs activity and } \\
\text { LUTS of participants. Raters were unaware of which group the participants be- } \\
\text { longed. }\end{array}$ \\
\hline $\begin{array}{l}\text { Incomplete outcome data } \\
\text { (attrition bias) } \\
\text { All outcomes }\end{array}$ & Unclear risk & $\begin{array}{l}\text { Randomised numbers in abstract (intervention } 16+\text { control } 15 \text { ) differ from } \\
\text { number in figure } 1 \text { (of publication) (intervention } 18+\text { control 17); not clear } \\
\text { when participants dropped out but reasons for attrition explained. }\end{array}$ \\
\hline $\begin{array}{l}\text { Selective reporting (re- } \\
\text { porting bias) }\end{array}$ & Unclear risk & $\begin{array}{l}\text { Table } 1 \text { (of publication) did not include the } 18 \text { and } 17 \text { randomised ( } 2 \text { complet- } \\
\text { ed the study but refused post-test outcome measuring). }\end{array}$ \\
\hline Other bias & Low risk & None detected. \\
\hline
\end{tabular}

Song 2013

\begin{tabular}{ll}
\hline Methods & Design: multicentre RCT \\
Study dates: July 2009 to August 2012 \\
Setting: China, inpatient and outpatient (4 centres) \\
\hline Participants \\
Number and \% women: intervention 82 (60.3\%), control 39 (57.4\%) \\
Age (mean): intervention 55 (SD 7) years, control 54 (SD 7) years \\
Diagnosed according to diagnostic criteria of 1995 National Meeting on Cerebrovascular Accidents and \\
meeting Urological criteria for incontinence. Type of stroke not reported. Participants had incontinence \\
with overflow and lack of voluntary control. \\
Duration of incontinence (mean): intervention 31.2 (SD 22.3) days, range 11-165 days; control 29.2 (SD \\
24.2) days, range 9-160 days.
\end{tabular}


Song 2013 (Continued)

Inclusion criteria: stroke with involuntary UI with overflow incontinence, stable condition, able to undergo investigations and treatment, no known cognitive impairment, aged $<70$ years, consenting.

Exclusion criteria: incontinence not arising from stroke, incontinence pre-existing prior to stroke, UTIs, cognitive impairment, severe heart/liver/kidney or other organ impairments, other reasons including fainting at needles, allergy, impaired clotting that would affect acupuncture, non-compliant to treatment, people with issues in diagnosis or measurements

Interventions

Intervention group: 136 participants. Acupuncture needles inserted at multiple acupuncture points (Qugu, Zhongji, Shuidao, Qihai, Baihui, Guanyuan, Sishenchong, Zhusanli, Sanyinjiao, etc.) on the arms, legs and torso. Stainless steel needles $0.3 \mathrm{~mm} \times 40 \mathrm{~mm}$ were disinfected and inserted $25 \mathrm{~mm}$, for 3 minutes and repeatedly inserted 3 times every 5 minutes. The Zhusanli, sanyingjiao were given current at 1-10 Hz, 2-5 mV, using a KWD808I machine, titrated up to participant's tolerance. Given 5 times per week, for 2 weeks per session. 2 sessions were given (i.e. 4 weeks)

Control group: 68 participants. Indwelling catheter placed with drainage every $2-4$ hours. Bladder washout performed once every 4 days. If any colour change or cloudiness noted, washing done twice daily until normal. Washout with $0.9 \%$ normal saline $250 \mathrm{~mL}$. If inflammatory appearance or having long-term indwelling catheter, gentamicin 160,000 units was given. Solutions were placed for 1.5-2 hours, before draining from bladder. Catheters changed every 2 weeks. Participants received bladder training in the morning, afternoon and evening: lower abdominal massage given (clockwise or anticlockwise motions 300-500 rubs), and also Kegel exercises 15-20 times, each time holding for 2-4 seconds. Catheters removed when the participants started to feel urge to pass urine. Total duration 4 weeks.

Outcomes Outcomes measured pre- and post-intervention (4 weeks)

Frequency, nocturia, intermittent stream, incontinence, postvoid volumes, participant satisfaction

\begin{tabular}{ll}
\hline Funding source & Not reported \\
\hline $\begin{array}{l}\text { Declarations of inter- } \\
\text { est among primary re- } \\
\text { searchers }\end{array}$ & None reported \\
\hline Notes & $\begin{array}{l}\text { Imbalance in number of participants in each group. Study reported in Chinese and translated into Eng- } \\
\text { lish. }\end{array}$ \\
\hline
\end{tabular}

\section{Risk of bias}

Bias Authors' judgement Support for judgement

Random sequence genera- Low risk Random number chart used.

tion (selection bias)

Allocation concealment $\quad$ Unclear risk $\quad$ Insufficient information to permit judgement.
(selection bias)

Blinding of participants Unclear risk Insufficient information to permit judgement.
and personnel (perfor-
mance bias)
All outcomes

\begin{tabular}{lll}
\hline $\begin{array}{l}\text { Blinding of outcome as- } \\
\text { sessment (detection bias) } \\
\text { All outcomes }\end{array}$ & Unclear risk & \\
\hline $\begin{array}{l}\text { Incomplete outcome data } \\
\text { (attrition bias) }\end{array}$ & Unclear risk & Insufficient information. Some unexplained attrition in both groups.
\end{tabular}


Song 2013 (Continued)

All outcomes

Selective reporting (re- Unclear risk $\quad$ No protocol available.
porting bias)

$\begin{array}{ll}\text { Other bias } \quad \text { Unclear risk } & \begin{array}{l}\text { Assessment made on translated information. Imbalance in number of partici- } \\ \text { pants in each group. }\end{array}\end{array}$

\begin{tabular}{l} 
Mesign: prospective, randomised controlled and single-blinded parallel trial \\
Study dates: recruited Jan 1999 to March 2001 \\
Setting: Copenhagen, Denmark. Outpatient Department of Geriatrics and Rheumatology \\
\hline
\end{tabular}

Participants 26 women stroke survivors with mixed stress/urge UI

Age (median): 60 (IQR 56-74) years

Time since stroke: intervention group median 12 months (interquartile range 2-20), control group median 13 months (interquartile range 2-50 months)

Inclusion criteria: women diagnosed with first ever ischaemic stroke according to WHO definition and verified by CAT scan, stroke symptoms in at least 1 month, normal cognitive function (Mini-Mental State Examination score <24), UI according to ICS definition, independent walking ability indoors $>100 \mathrm{~m}$ with or without aids, independence in toilet visits, aged $40-85$ years.

Exclusion criteria: UTI, symptom of vaginal prolapse, chronic respiratory diseases, psychiatric diseases, other neurological diseases, unable to speak Danish.

Interventions

Intervention group: 14 participants. Systematic, controlled, intensive PFM training programme. Programme consisted of: introduction to theory; home exercises, including strength PFM exercise by performing close to maximum contraction (6-second contraction and 6 -second rest), participants instructed to repeat exercise programme gradually $6-10$ times in supine, standing and sitting positions, 1 or 2 times daily; group treatment once per week, including isolating PFM contraction (6-second contraction and 6-second rest) and strength exercises (3-second contraction and 3-second rest, and 6-second contraction and 6-second rest). All techniques were repeated 4-8 times while supine, sitting and standing, and also prior to daily activities such as rising, sitting and walking. Vaginal palpation performed to ensure correct contraction, to evaluate contraction strength and to give feedback to participants.

Control group: 12 participants. Normal, general rehabilitation with no specific treatment of UI.

Outcomes

Voiding diary measured: 2-day mean voiding frequency over 24 hours; 2 -day mean daytime voiding frequency; 2-day mean night-time voiding frequency; 3-day mean voiding frequency over 24 hours; 3-day mean daytime voiding frequency; 3-day mean night-time voiding frequency (post-intervention)

24 hour pad test measured: UI: 2-day mean number of incontinence episodes per 24 hours; 2-day mean number of used pads per 24 hours; 3-day mean number of incontinence episodes per 24 hours; 3-day mean number of used pads per 24 hours (post-intervention)

Vaginal palpation measured PFM function: mean function of PFM; mean strength of PFM; mean static endurance of PFM; mean dynamic endurance of PFM (post-intervention)

Quality of life measured by: SF-36 and IIQ (post-intervention and 6-month follow-up)

Funding source

Grant sponsors: The Foundation of Danish Physiotherapists Research; The Foundation of 1870, Direktor Jacob Madsen og hustrus Fond 
Tibaek 2005 (Continued)

Declarations of interest among primary re-

None reported

searchers

Notes No baseline differences in age, number of births or mobility between groups.

Proportions for which data reported: $92 \%$ at post-intervention and 6 months

\section{Risk of bias}

\begin{tabular}{lll}
\hline Bias & Authors' judgement & Support for judgement \\
\hline $\begin{array}{l}\text { Random sequence genera- } \\
\text { tion (selection bias) }\end{array}$ & Low risk & $\begin{array}{l}\text { Randomised procedure using a mathematical table, conducted by a physio- } \\
\text { therapist who had no further involvement in study. }\end{array}$ \\
\hline $\begin{array}{l}\text { Allocation concealment } \\
\text { (selection bias) }\end{array}$ & Unclear risk & $\begin{array}{l}\text { Used sealed numbered envelopes but not stated if they were sequentially } \\
\text { numbered and opaque. }\end{array}$ \\
\hline $\begin{array}{l}\text { Blinding of participants } \\
\text { and personnel (perfor- } \\
\text { mance bias) }\end{array}$ & Low risk & $\begin{array}{l}\text { Participants not blinded. Physiotherapist delivering the 12-week intervention } \\
\text { not blinded. Outcome not likely to be influenced by lack of blinding. }\end{array}$ \\
\hline
\end{tabular}

\begin{tabular}{|c|c|c|}
\hline $\begin{array}{l}\text { Blinding of outcome as- } \\
\text { sessment (detection bias) } \\
\text { All outcomes }\end{array}$ & Unclear risk & Not described. \\
\hline \multirow[t]{4}{*}{$\begin{array}{l}\text { Incomplete outcome data } \\
\text { (attrition bias) } \\
\text { All outcomes }\end{array}$} & Unclear risk & $\begin{array}{l}\text { Attrition reasons reported. Reasons for missing data reported for participant } \\
\text { administered variables ( } 0 \% \text { to } 21 \%) \text {. Reasons not provided for missing data for } \\
\text { investigator administered variables ( } 0 \% \text { to } 4 \% \text { ). }\end{array}$ \\
\hline & & Figure 2 (of publication): not clear how many participants the results were for. \\
\hline & & $\begin{array}{l}\text { Figure } 3 \text { (of publication): not clear if the participant unable to do pretest PFM } \\
\text { was included in figure or excluded. }\end{array}$ \\
\hline & & Table V (of publication): only 11 intervention participants reported. \\
\hline $\begin{array}{l}\text { Selective reporting (re- } \\
\text { porting bias) }\end{array}$ & Unclear risk & Protocol not available. \\
\hline Other bias & Low risk & None detected. \\
\hline
\end{tabular}

Tibaek 2017

$\begin{array}{ll}\text { Methods } & \text { Design: experimental prospective RCT using randomised, controlled and single-blinded parallel groups } \\ \text { Setting: Denmark, Outpatients }\end{array}$

Participants 31 men

\footnotetext{
Age (mean): overall 68 years; intervention 68 (range 57-73) years, control: 70 (range 64-75) years.

Description of stroke and UI:

Ischaemic stroke: intervention 13 (87\%), control 10 (76\%)

Haemorrhagic stroke: intervention 0, control 2 (13\%)
} 
Tibaek 2017 (Continued)

Time from stroke to trial entry (median): intervention 65 (IQR 50-87) days; control 55 (IQR 50-63) days.

Inclusion criteria: men, diagnosed with stroke clinically defined according to the WHO (1989) and confirmed by CT or MRI scan; $\geq 1$ month since their last stroke; normal cognitive function (Mini-Mental State Examination score > 25); LUTS according to the ICS definition (Abrams 2002) with start or aggravation closely related to stroke and measured by DAN-PSS-1 questionnaire; ability to walk independently indoors for at least $100 \mathrm{~m}$ with or without walking aids; outpatient and self-transported; ability to visit the toilet independently aged $>18$ years.

Exclusion criteria: history of LUTS surgery, pelvic surgery or trauma; > 2 strokes (diagnosed at a hospital); other severe neurological disease including dementia; severe dysphasia; severe psychiatric disease; prostate cancer and not speaking Danish or English.

Interventions

Intervention: 16 participants. Systematic, controlled, intensive PFMT programme over 3 months (12 weekly sessions and 60 minutes per session) by the same specialised physiotherapist. The treatment programme consisted of the following topics: introduction (theory), home exercise, group treatment and digital anal palpation of PFMs.

Control: 15 participants. Standard programme of general rehabilitation without any specific treatment for LUTS.

Outcomes

Outcomes measured pretest, post-test (12 weeks) and at 6-month follow-up.

Primary outcomes: DAN-PSS-1 questionnaire, 3-day voiding diary

Secondary outcomes: digital anal palpation of PFM

Health status: SF-36.

Funding source

The authors disclosed receipt of the following financial support for the research, authorship, publication (or a combination) of this article: "This study was supported by grants from the Association of Danish Physiotherapists' Research Foundation and Practice Foundation, the Foundation of 17.12.1981, Lykkefeldts Grant, the Foundation of Lundbeck (UCSF) and the Department of Physiotherapy and Occupational Therapy Glostrup Hospital, University of Copenhagen."

Declarations of interest among primary researchers
Authors declared no potential conflicts of interest with respect to the research, authorship, publication (or a combination) of this article.

Notes

\section{Risk of bias}

\begin{tabular}{|c|c|c|}
\hline Bias & Authors' judgement & Support for judgement \\
\hline $\begin{array}{l}\text { Random sequence genera- } \\
\text { tion (selection bias) }\end{array}$ & Low risk & $\begin{array}{l}\text { Randomisation based on a mathematical table delivered in blocks of } 10 \text { in } \\
\text { sealed envelopes and managed by a person who did not participate further in } \\
\text { study. }\end{array}$ \\
\hline $\begin{array}{l}\text { Allocation concealment } \\
\text { (selection bias) }\end{array}$ & Unclear risk & Unclear if envelopes were opaque. \\
\hline $\begin{array}{l}\text { Blinding of participants } \\
\text { and personnel (perfor- } \\
\text { mance bias) } \\
\text { All outcomes }\end{array}$ & Low risk & $\begin{array}{l}\text { Registration of variables from the pretest, post-test and 6-month follow-up in- } \\
\text { vestigation sequences were managed by physiotherapists who were blinded } \\
\text { to the randomisation code of the participants, while the physiotherapist treat- } \\
\text { ing the participants with PFMT was unblinded, Outcome not likely to be affect- } \\
\text { ed by this lack of blinding. }\end{array}$ \\
\hline
\end{tabular}


Tibaek 2017 (Continued)

\begin{tabular}{|c|c|c|}
\hline $\begin{array}{l}\text { Blinding of outcome as- } \\
\text { sessment (detection bias) } \\
\text { All outcomes }\end{array}$ & Low risk & $\begin{array}{l}\text { Registration of variables from the pretest, post-test and follow-up investiga- } \\
\text { tion sequences were managed by physiotherapists who were blinded to the } \\
\text { randomisation code of participants. }\end{array}$ \\
\hline
\end{tabular}

\begin{tabular}{lll}
\hline $\begin{array}{l}\text { Incomplete outcome data } \\
\text { (attrition bias) } \\
\text { All outcomes }\end{array}$ & Low risk & $\begin{array}{l}\text { One dropout after randomisation in intervention group. At 6-month follow-up, } \\
1 \text { participant from the control group dropped out. No reasons provided. }\end{array}$ \\
\hline $\begin{array}{l}\text { Selective reporting (re- } \\
\text { porting bias) }\end{array}$ & Unclear risk & Protocol not available. \\
\hline Other bias & Low risk & None detected. \\
\hline
\end{tabular}

\section{Zhang 1996}

$\begin{array}{ll}\text { Methods } & \text { Design: RCT } \\ \text { Study dates: not reported } \\ \text { Setting: China, hospital Department of Internal Medicine }\end{array}$

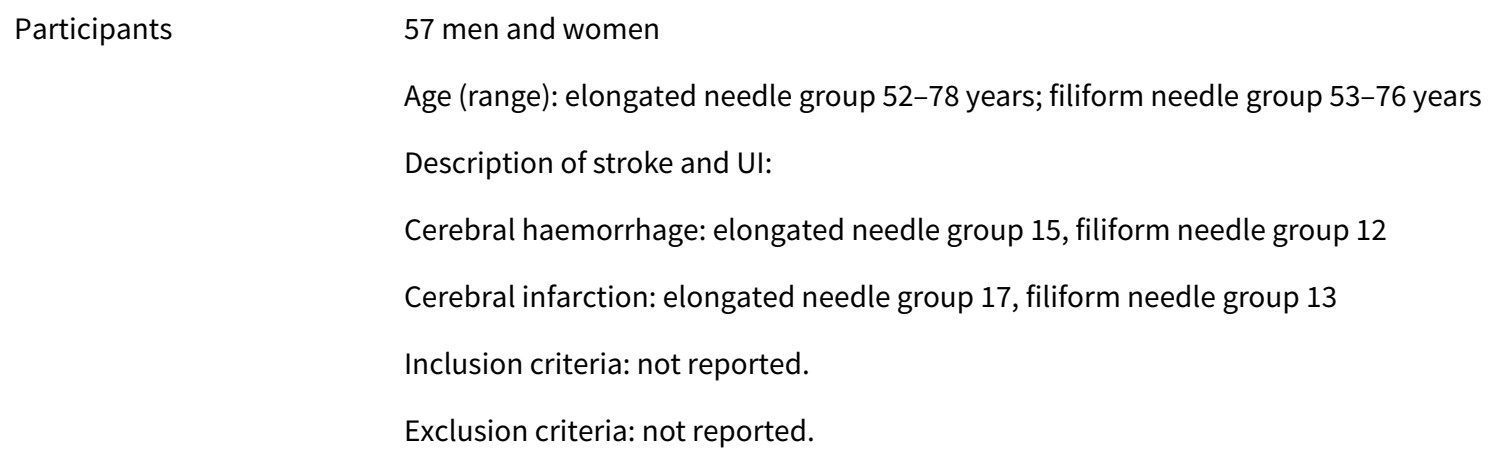
grees with the skin, twirled and pushed forward slowly to the greater sciatic foramen. When the needle was inserted into a depth of about 5 Cun and if the participant had sensations of tightness and distension, the insertion was stopped immediately. This meant that the needle tip reached the back of the rectum and the front of the sacrum. The needle was twirled in 1 direction and manipulated with the uniform reinforcing and reducing method, causing the participant to have enough needling sensation and feelings of raising and contraction in the anus, which displays the success of needling. The needle was manipulated once every 10 minutes and retained for 50 minutes.

Filiform needle group: 25 participants. Lateral position. After local routine sterilisation, Guanyan (CV 4), Zhongji (CV 3), Sanyinjiao (SP 6, both), Shenshu (BL 23) and Pangguangshu (BL 28) were punctured with filiform needles 2 Cun long which advanced to a depth of about 1.5 Cun. Following needling sensations achieved, needles were manipulated with the uniform reinforcing and reducing method and then retained for 50 minutes.

All participants treated once daily. 6 days formed a therapeutic course, with 1 day of rest between 2 courses. Total of 4 courses of treatment given.

Outcomes Outcome measured after 4 courses of treatment (28 days).

Continent/incontinent, improvement in urinary symptoms. 
Zhang 1996 (Continued)

\begin{tabular}{ll} 
Funding source & Not reported \\
\hline $\begin{array}{l}\text { Declarations of inter- } \\
\text { est among primary re- }\end{array}$ & Not reported \\
searchers &
\end{tabular}

Notes

\section{Risk of bias}

\begin{tabular}{|c|c|c|}
\hline Bias & Authors' judgement & Support for judgement \\
\hline $\begin{array}{l}\text { Random sequence genera- } \\
\text { tion (selection bias) }\end{array}$ & Unclear risk & Quote: "randomly divided." \\
\hline $\begin{array}{l}\text { Allocation concealment } \\
\text { (selection bias) }\end{array}$ & Unclear risk & Method of concealment not described. \\
\hline $\begin{array}{l}\text { Blinding of participants } \\
\text { and personnel (perfor- } \\
\text { mance bias) } \\
\text { All outcomes }\end{array}$ & Unclear risk & Not reported. \\
\hline $\begin{array}{l}\text { Blinding of outcome as- } \\
\text { sessment (detection bias) } \\
\text { All outcomes }\end{array}$ & Unclear risk & Not reported. \\
\hline $\begin{array}{l}\text { Incomplete outcome data } \\
\text { (attrition bias) } \\
\text { All outcomes }\end{array}$ & Low risk & Outcome data available for all participants. \\
\hline $\begin{array}{l}\text { Selective reporting (re- } \\
\text { porting bias) }\end{array}$ & Unclear risk & No protocol available. \\
\hline Other bias & High risk & $\begin{array}{l}\text { No baseline assessment; unable to assess similarity of participants between } \\
\text { groups. }\end{array}$ \\
\hline
\end{tabular}

Zhang 2002

\begin{tabular}{ll} 
Methods & Design: RCT \\
& Study dates: January 1999 to December 2001 \\
& Setting: unclear \\
\hline Participants & 38 male (59\%) and 26 female (41\%) adult stroke survivors with UI \\
& Age (range): $42-62$ years \\
& $\begin{array}{l}29 \text { participants had multiple cerebral infarctions; } 14 \text { had left-side basal ganglia infarction; } 9 \text { had right- } \\
\text { side basal ganglia infarction; } 5 \text { had frontal lobe infarction; } 6 \text { had parietal, occipital or temporal lobe in- } \\
\text { farction; } 1 \text { had cerebellum infarction. } \\
\text { Inclusion and exclusion: not reported }\end{array}$ \\
\hline
\end{tabular}


Zhang 2002 (Continued) Interventions

Intervention group: 36 participants. Acupuncture at the Tsusanli, Yinlingquan and Sanyinjiao points using 3-inch needle to acupuncture at the depth of 2-2.25 inches, 20 minutes per session, once per day for 7 times in the treatment period.

Control group: 28 participants. Mannite and other medicines to do general treatment with the partial foment on the bladder.

\section{Time points not reported}

Numbers not regaining continence

\begin{tabular}{ll}
\hline Funding source & Not reported \\
\hline $\begin{array}{l}\text { Declarations of inter- } \\
\text { est among primary re- } \\
\text { searchers }\end{array}$ & Not reported \\
\hline Notes & Study reported in Chinese and translated into English. \\
& No description of baseline comparisons. \\
& Trial was reported in a conference abstract. \\
\hline
\end{tabular}

\section{Risk of bias}

\begin{tabular}{lll}
\hline Bias & Authors' judgement & Support for judgement \\
\hline $\begin{array}{l}\text { Random sequence genera- } \\
\text { tion (selection bias) }\end{array}$ & Unclear risk & Information not provided. \\
\hline $\begin{array}{l}\text { Allocation concealment } \\
\text { (selection bias) }\end{array}$ & Unclear risk & Information not provided. \\
\hline
\end{tabular}

Blinding of participants Unclear risk Information not provided.

and personnel (perfor-

mance bias)

All outcomes

Blinding of outcome as- Unclear risk Information not provided.

sessment (detection bias)

All outcomes

Incomplete outcome data Low risk Results suggested all participants accounted for and included in analysis.
(attrition bias)

(attrition bias)

All outcomes

Selective reporting (re- Unclear risk $\quad$ Protocol not available.
porting bias)

Other bias Unclear risk Insufficient information reported/translated.

Zhou 1999

$\begin{array}{ll}\text { Methods } & \text { Design: RCT } \\ & \text { Study dates: } 1995\end{array}$


Zhou 1999 (Continued)

Setting: unclear

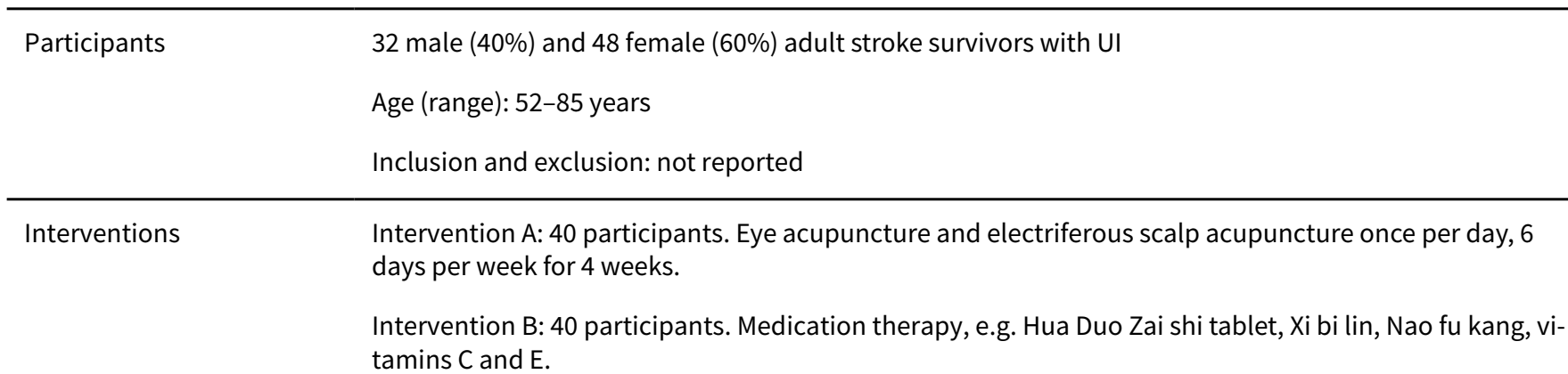

\begin{tabular}{ll}
\hline Outcomes & Numbers not regaining continence \\
\hline Funding source & Not reported \\
\hline
\end{tabular}

Declarations of inter- Not reported

est among primary re-

searchers

\begin{tabular}{ll}
\hline Notes & Study reported in Chinese and translated into English. \\
No description of baseline comparisons. \\
Trial reported in a conference abstract. \\
\hline
\end{tabular}

\section{Risk of bias}

\begin{tabular}{lll}
\hline Bias & Authors' judgement & Support for judgement \\
\hline $\begin{array}{l}\text { Random sequence genera- } \\
\text { tion (selection bias) }\end{array}$ & Unclear risk & $\begin{array}{l}\text { Quote: "Patients are allocated using a single-blind randomisation to the two } \\
\text { groups." } \\
\text { Comment: no further description. }\end{array}$ \\
\hline $\begin{array}{l}\text { Allocation concealment } \\
\text { (selection bias) }\end{array}$ & Unclear risk & $\begin{array}{l}\text { Quote: "Patients are allocated using a single-blind randomisation to the two } \\
\text { groups." }\end{array}$ \\
\hline $\begin{array}{l}\text { Blinding of participants } \\
\text { and personnel (perfor- } \\
\text { mance bias) }\end{array}$ & Unclear risk & $\begin{array}{l}\text { Comment: no further description. } \\
\text { All outcomes }\end{array}$ \\
\hline
\end{tabular}

\begin{tabular}{lll}
\hline $\begin{array}{l}\text { Blinding of outcome as- } \\
\text { sessment (detection bias) } \\
\text { All outcomes }\end{array}$ & Unclear risk & No description provided. \\
\hline $\begin{array}{l}\text { Incomplete outcome data } \\
\text { (attrition bias) } \\
\text { All outcomes }\end{array}$ & Low risk & Data presented appeared complete. \\
\hline $\begin{array}{l}\text { Selective reporting (re- } \\
\text { porting bias) }\end{array}$ & Unclear risk & No protocol available. \\
\hline \begin{tabular}{l} 
Other bias \\
\hline \hline
\end{tabular}
\end{tabular}


AUA: American Urological Association; CAT: computed axial tomography; CNP: continence nurse practitioner; CT: computed tomography; CTU: Clinical Trials Unit; DAN-PSS: Danish Prostatic Symptom Score; EQ-5D-5L: EuroQol Five-Dimensional Questionnaire; GP: general practitioner; ICIQ-UI-SF: International Consultation on Incontinence Questionnaire - Urinary Incontinence Short Form; ICIQLUTSqol: International Consultation on Incontinence Questionnaire - Lower Urinary Tract Symptoms Quality of Life questionnaire; ICS: International Continence Society; IIQ: Incontinence Impact Questionnaire; IQR: interquartile range; LUTS: lower urinary tract symptom; MRI: magnetic resonance imaging; NHS: National Health Service; OABSS: Overactive Bladder Symptom Score; PFM: pelvic floor muscle; PFMT: pelvic floor muscle training; RCT: randomised controlled trial; SD: standard deviation; SF-36: 36-Item Short Form; SUFA: Society of Urodynamics; TENS: transcutaneous electrical nerve stimulation; TPTNS: transcutaneous posterior tibial nerve stimulation; UI: urinary incontinence; UTI: urinary tract infection; WHO: World Health Organization.

\section{Characteristics of excluded studies [ordered by study ID]}

\begin{tabular}{|c|c|}
\hline Study & Reason for exclusion \\
\hline Byles 2006 & Study terminated due to poor recruitment. Study authors stated the study was abandoned. \\
\hline Cook 1998 & Study did not include a measure of continence. \\
\hline Engberg 2002 & People with stroke were a subgroup but data not reported or available. \\
\hline EUCTR2009-009216-53-PT & Study did not include people with stroke. \\
\hline Gong 2013 & $\begin{array}{l}\text { Participants recruited were in the acute phase of stroke, which was not the type of participants un- } \\
\text { der investigation. }\end{array}$ \\
\hline Gousse 2007 & Did not include people with stroke. \\
\hline Gross 1990 & Did not include a measure of continence. \\
\hline Humphreys 2014 & Did not include a measure of continence. \\
\hline ISRCTN97151578 & Unable to trace author or publications. \\
\hline Kim 2003 & Did not include people with stroke. \\
\hline Kuo 2007 & People with stroke were a subgroup but data not reported or available. \\
\hline Madersbacher 2004 & Did not include people with stroke. \\
\hline Madersbacher 2005 & Did not include people with stroke. \\
\hline McDowell 1999 & People with stroke were a subgroup but data not reported or available. \\
\hline Moon 2012 & Did not include a measure of continence \\
\hline NCT00213577 & People with stroke were a subgroup but data not reported or available. \\
\hline NCT01275261 & Study terminated. No data analysed (ClinicalTrials.gov). \\
\hline Sakakibara 2008 & People with stroke were a subgroup but data not reported or available. \\
\hline Shin $2016 c$ & Study terminated due to poor recruitment (ClinicalTrials.gov (NCT02472288)) \\
\hline Smilskalne 2009 & $\begin{array}{l}\text { Abstract of a completed study. No statistical data reported. We were unable to locate further publi- } \\
\text { cations from the study and were unable to trace the author for further details. }\end{array}$ \\
\hline Stohrer 2013 & People with stroke were a subgroup but data not reported or available. \\
\hline
\end{tabular}




\begin{tabular}{ll}
\hline Study & Reason for exclusion \\
\hline Tekeoglu 1998 & Did not directly test a method of promoting continence. \\
\hline Thomas 2011 & $\begin{array}{l}\text { Participants recruited were in the acute phase of stroke, which was not the type of participants un- } \\
\text { der investigation. }\end{array}$ \\
\hline Vinsnes 2010 & Did not include people with stroke. \\
\hline Wikander 1998 & $\begin{array}{l}\text { Participants recruited were in the acute phase of stroke, which was not the type of participants un- } \\
\text { der investigation. }\end{array}$ \\
\hline Yun 2007 & $\begin{array}{l}\text { Participants recruited were in the acute phase of stroke, which was not the type of participants un- } \\
\text { der investigation. Study did not include a measure of continence. }\end{array}$ \\
\hline Zhu 2003 & $\begin{array}{l}\text { Participants recruited were in the acute phase of stroke, which was not the type of participants un- } \\
\text { der investigation. }\end{array}$ \\
\hline
\end{tabular}

\section{Characteristics of studies awaiting assessment [ordered by study ID]}

\section{ACTRN12617000162314}

\begin{tabular}{ll}
\hline Methods & RCT \\
\hline Participants & Ischaemic stroke $\geq 6$ months previously with UI, women, aged 40-75 years \\
\hline Interventions & PFMT vs usual care \\
\hline Outcomes & Primary outcome: resting and functional bioelectrical activity of PFM \\
& Secondary outcome: subjective symptoms of UI by ICIQ \\
\hline Notes & Not yet recruited to trial. \\
\hline
\end{tabular}

\section{ChiCTR-INR-16010239}

\begin{tabular}{ll}
\hline Methods & Randomised parallel controlled trial \\
\hline Participants & Aged 40-75 years with UI post-stroke. Onset of stroke within 30 days. \\
\hline Interventions & Acupuncture vs computer intermediate frequency electrotherapy \\
\hline Outcomes & Residual urine volume, voided volume, urination time \\
\hline Notes & Estimated trial completion date: 31 December 2018 \\
\hline
\end{tabular}

Wang 2014

\begin{tabular}{ll}
\hline Methods & Multicentre, randomised, single-blind controlled clinical trial \\
\hline Participants & $\begin{array}{l}\text { Adults aged } 40-75 \text { years, with urge UI occurring 4-48 weeks after stroke, diagnostic criteria accord- } \\
\text { ing to ASA and ICS }\end{array}$
\end{tabular}


Wang 2014 (Continued)

Interventions

\section{Outcomes}

Traditional moxibustion therapy + routine care vs new type moxibustion therapy + routine care vs routine care

Notes Trial completed, results not published

ASA: American Stroke Association; ICIQ: International Consultation on Incontinence Questionnaire; ICS: International Continence Society; PFMT: pelvic floor muscle training; QoL: quality of life; RCT: randomised controlled trial; UI: urinary incontinence.

Characteristics of ongoing studies [ordered by study ID]

\section{NCT02568774}

Trial name or title

Methods RCT

\begin{tabular}{ll}
\hline Participants & Aged $\geq 18$ years, first or recurrent stroke with no previous incontinence \\
\hline Interventions & Acupuncture vs usual care \\
\hline Outcomes & Incontinent episodes, frequency daytime, frequency night-time, SS-QOL \\
\hline Starting date & August 2015 \\
\hline Contact information & yeungwfj@hk.hk \\
\hline Notes & Estimated completion date: July 2018 \\
\hline
\end{tabular}

Shin 2016b

Trial name or title

Effectiveness and safety of electro-acupuncture on post-stroke urinary incontinence: study protocol of a pilot multicentered, randomized, parallel, sham-controlled trial

\begin{tabular}{ll}
\hline Methods & Pilot multicentred, randomised, parallel, sham-controlled trial \\
\hline Participants & $\begin{array}{l}\text { Aged } \geq 19 \text { years within } 2 \text { years of stroke occurrence, with } \geq 2 \text { urinary frequencies and scoring } 3 \text { or } 4 \\
\text { points on the PPIUS or scoring } 13 \text { points on the K-IPSS }\end{array}$ \\
\hline Interventions & Electroacupuncture vs sham therapy \\
\hline Outcomes & Primary outcome: TUFS \\
\hline Starting date & Secondary outcomes: QoL (ICIQ-UI-SF), K-IPSS, LUTS \\
\hline Contact information & June 2016 \\
\hline Notes & mapsi@daum.net \\
\hline
\end{tabular}


ICIQ-UI-SF: International Consultation on Incontinence Questionnaire - Urinary Incontinence Short Form; LUTS: lower urinary tract symptoms; K-IPSS: Korean version of International Prostate Symptom Score; PPIUS: Patient Perception of Intensity of Urgency Scale; RCT: randomised controlled trial; SS-QOL: Stroke Specific Quality of Life Scale; TUFS: total urgency and frequency score.

\section{DATA AND ANALYSES}

\section{Comparison 1. Intervention versus no intervention/usual care}

\begin{tabular}{|c|c|c|c|c|}
\hline Outcome or subgroup title & No. of studies & $\begin{array}{l}\text { No. of partici- } \\
\text { pants }\end{array}$ & Statistical method & Effect size \\
\hline $\begin{array}{l}1 \text { Number of participants continent af- } \\
\text { ter treatment }\end{array}$ & 6 & & $\begin{array}{l}\text { Risk Ratio (M-H, Random, } \\
95 \% \mathrm{Cl})\end{array}$ & Subtotals only \\
\hline 1.1 Professional input interventions & 1 & 121 & $\begin{array}{l}\text { Risk Ratio (M-H, Random, } \\
95 \% \mathrm{Cl})\end{array}$ & $1.28[0.81,2.02]$ \\
\hline $\begin{array}{l}1.2 \text { Complementary therapy interven- } \\
\text { tions }\end{array}$ & 5 & 524 & $\begin{array}{l}\text { Risk Ratio (M-H, Random, } \\
95 \% \mathrm{Cl})\end{array}$ & $2.82[1.57,5.07]$ \\
\hline $\begin{array}{l}2 \text { Number of incontinent episodes in } 24 \\
\text { hours (mean) }\end{array}$ & 4 & & $\begin{array}{l}\text { Mean Difference (IV, Random, } \\
95 \% \mathrm{CI} \text { ) }\end{array}$ & Subtotals only \\
\hline 2.1 Behavioural interventions & 1 & 18 & $\begin{array}{l}\text { Mean Difference (IV, Random, } \\
95 \% \mathrm{CI})\end{array}$ & $-1.0[-2.74,0.74]$ \\
\hline 2.2 Physical therapy interventions & 3 & 142 & $\begin{array}{l}\text { Mean Difference (IV, Random, } \\
95 \% \mathrm{CI})\end{array}$ & $\begin{array}{l}-4.76[-8.10 \\
-1.41]\end{array}$ \\
\hline $\begin{array}{l}3 \text { Number of participants cured of all } \\
\text { four urinary symptoms }\end{array}$ & 1 & & $\begin{array}{l}\text { Risk Ratio (M-H, Fixed, 95\% } \\
\mathrm{Cl})\end{array}$ & Subtotals only \\
\hline $\begin{array}{l}\text { 3.1 Specialised professional input in- } \\
\text { terventions }\end{array}$ & 1 & 143 & $\begin{array}{l}\text { Risk Ratio (M-H, Fixed, 95\% } \\
\mathrm{Cl})\end{array}$ & $1.55[0.97,2.48]$ \\
\hline $\begin{array}{l}4 \text { Urinary symptoms - frequency (con- } \\
\text { tinuous variables) }\end{array}$ & 6 & & $\begin{array}{l}\text { Mean Difference (IV, Random, } \\
95 \% \mathrm{CI})\end{array}$ & Subtotals only \\
\hline 4.1 Behavioural interventions & 2 & 48 & $\begin{array}{l}\text { Mean Difference (IV, Random, } \\
95 \% \mathrm{CI})\end{array}$ & $\begin{array}{l}-1.71[-3.02 \\
-0.40]\end{array}$ \\
\hline $\begin{array}{l}4.2 \text { Complementary therapy interven- } \\
\text { tions }\end{array}$ & 1 & 62 & $\begin{array}{l}\text { Mean Difference (IV, Random, } \\
95 \% \mathrm{CI} \text { ) }\end{array}$ & $\begin{array}{l}-5.57[-7.00 \\
-4.14]\end{array}$ \\
\hline 4.3 Physical therapy interventions & 3 & 142 & $\begin{array}{l}\text { Mean Difference (IV, Random, } \\
95 \% \mathrm{CI})\end{array}$ & $-2.83[-5.75,0.09]$ \\
\hline $\begin{array}{l}5 \text { Urinary symptoms - frequency (di- } \\
\text { chotomous variables) }\end{array}$ & 1 & & $\begin{array}{l}\text { Risk Ratio (M-H, Fixed, 95\% } \\
\mathrm{Cl})\end{array}$ & Subtotals only \\
\hline $\begin{array}{l}5.1 \text { Specialised professional input in- } \\
\text { terventions }\end{array}$ & 1 & 187 & $\begin{array}{l}\text { Risk Ratio (M-H, Fixed, 95\% } \\
\mathrm{Cl})\end{array}$ & $0.93[0.82,1.05]$ \\
\hline 6 Urinary symptoms - urgency & 1 & & $\begin{array}{l}\text { Risk Ratio (M-H, Fixed, 95\% } \\
\mathrm{Cl})\end{array}$ & Subtotals only \\
\hline
\end{tabular}




\begin{tabular}{|c|c|c|c|c|}
\hline Outcome or subgroup title & No. of studies & $\begin{array}{l}\text { No. of partici- } \\
\text { pants }\end{array}$ & Statistical method & Effect size \\
\hline $\begin{array}{l}\text { 6.1 Specialised professional input in- } \\
\text { terventions }\end{array}$ & 1 & 188 & $\begin{array}{l}\text { Risk Ratio (M-H, Fixed, 95\% } \\
\mathrm{Cl})\end{array}$ & $1.05[0.89,1.24]$ \\
\hline $\begin{array}{l}7 \text { Urinary symptoms - nocturia (contin- } \\
\text { uous variables) }\end{array}$ & 5 & & $\begin{array}{l}\text { Mean Difference (IV, Random, } \\
95 \% \mathrm{CI} \text { ) }\end{array}$ & Subtotals only \\
\hline 7.1 Behavioural interventions & 2 & 48 & $\begin{array}{l}\text { Mean Difference (IV, Random, } \\
95 \% \mathrm{CI} \text { ) }\end{array}$ & $-0.38[-1.06,0.29]$ \\
\hline $\begin{array}{l}7.2 \text { Complementary therapy interven- } \\
\text { tions }\end{array}$ & 2 & 256 & $\begin{array}{l}\text { Mean Difference (IV, Random, } \\
95 \% \mathrm{CI} \text { ) }\end{array}$ & $-1.76[-4.49,0.96]$ \\
\hline 7.3 Physical therapy interventions & 1 & 61 & $\begin{array}{l}\text { Mean Difference (IV, Random, } \\
95 \% \mathrm{Cl} \text { ) }\end{array}$ & $\begin{array}{l}-1.52[-1.73 \\
-1.31]\end{array}$ \\
\hline $\begin{array}{l}8 \text { Urinary symptoms - nocturia (di- } \\
\text { chotomous variables) }\end{array}$ & 1 & & $\begin{array}{l}\text { Risk Ratio (M-H, Fixed, 95\% } \\
\mathrm{Cl})\end{array}$ & Subtotals only \\
\hline $\begin{array}{l}8.1 \text { Specialised professional input in- } \\
\text { terventions }\end{array}$ & 1 & 186 & $\begin{array}{l}\text { Risk Ratio (M-H, Fixed, 95\% } \\
\mathrm{Cl})\end{array}$ & $0.96[0.86,1.07]$ \\
\hline $\begin{array}{l}9 \text { Physical measures: mean function of } \\
\text { the pelvic floor muscle }\end{array}$ & 1 & & $\begin{array}{l}\text { Mean Difference (IV, Fixed, } \\
95 \% \mathrm{CI} \text { ) }\end{array}$ & Subtotals only \\
\hline 9.1 Behavioural interventions & 1 & 23 & $\begin{array}{l}\text { Mean Difference (IV, Fixed, } \\
95 \% \mathrm{Cl} \text { ) }\end{array}$ & $0.10[-0.48,0.68]$ \\
\hline $\begin{array}{l}10 \text { Health status and quality of life - } \\
\text { health status, mean total score } 36 \text {-Item } \\
\text { Short Form }\end{array}$ & 1 & & $\begin{array}{l}\text { Mean Difference (IV, Fixed, } \\
95 \% \mathrm{Cl} \text { ) }\end{array}$ & Subtotals only \\
\hline 10.1 Behavioural interventions & 1 & 24 & $\begin{array}{l}\text { Mean Difference (IV, Fixed, } \\
95 \% \mathrm{CI})\end{array}$ & $\begin{array}{l}-28.0[-169.66 \\
113.66]\end{array}$ \\
\hline $\begin{array}{l}11 \text { Health status and quality of life - } \\
\text { quality of life }\end{array}$ & 2 & & $\begin{array}{l}\text { Std. Mean Difference (IV, Ran- } \\
\text { dom, } 95 \% \mathrm{CI} \text { ) }\end{array}$ & Subtotals only \\
\hline 11.1 Behavioural interventions & 2 & 55 & $\begin{array}{l}\text { Std. Mean Difference (IV, Ran- } \\
\text { dom, } 95 \% \mathrm{Cl} \text { ) }\end{array}$ & $-0.99[-2.83,0.86]$ \\
\hline $\begin{array}{l}12 \text { Functional ability - mean Barthel } \\
\text { score (continuous variables) }\end{array}$ & 2 & & $\begin{array}{l}\text { Mean Difference (IV, Random, } \\
95 \% \mathrm{Cl} \text { ) }\end{array}$ & Subtotals only \\
\hline 12.1 Physical therapy interventions & 2 & 81 & $\begin{array}{l}\text { Mean Difference (IV, Random, } \\
95 \% \mathrm{Cl} \text { ) }\end{array}$ & $8.97[1.27,16.68]$ \\
\hline $\begin{array}{l}13 \text { Participant satisfaction - numbers } \\
\text { who were dissatisfied }\end{array}$ & 1 & & $\begin{array}{l}\text { Risk Ratio (M-H, Fixed, 95\% } \\
\mathrm{Cl})\end{array}$ & Subtotals only \\
\hline $\begin{array}{l}13.1 \text { Specialised professional input in- } \\
\text { terventions }\end{array}$ & 1 & 154 & $\begin{array}{l}\text { Risk Ratio (M-H, Fixed, 95\% } \\
\text { Cl) }\end{array}$ & $0.32[0.17,0.59]$ \\
\hline
\end{tabular}


Analysis 1.1. Comparison 1 Intervention versus no intervention/ usual care, Outcome 1 Number of participants continent after treatment.

\begin{tabular}{|c|c|c|c|c|c|}
\hline Study or subgroup & $\begin{array}{c}\text { Treatment } \\
\mathrm{n} / \mathrm{N} \\
\end{array}$ & $\begin{array}{c}\text { Control } \\
\mathrm{n} / \mathrm{N}\end{array}$ & $\begin{array}{c}\text { Risk Ratio } \\
\text { M-H, Random, } 95 \% \text { Cl }\end{array}$ & Weight & $\begin{array}{c}\text { Risk Ratio } \\
\text { M-H, Random, } 95 \% \mathrm{Cl}\end{array}$ \\
\hline \multicolumn{6}{|c|}{ 1.1.1 Professional input interventions } \\
\hline Brittain 2000b & $33 / 73$ & $17 / 48$ & & $100 \%$ & $1.28[0.81,2.02]$ \\
\hline Subtotal $(95 \% \mathrm{Cl})$ & 73 & 48 & & $100 \%$ & $1.28[0.81,2.02]$ \\
\hline \multicolumn{6}{|c|}{ Total events: 33 (Treatment), 17 (Control) } \\
\hline \multicolumn{6}{|c|}{ Heterogeneity: Not applicable } \\
\hline \multicolumn{6}{|c|}{ 1.1.2 Complementary therapy interventions } \\
\hline Chu 2011 & $16 / 56$ & $3 / 55$ & $\longrightarrow$ & $13.87 \%$ & $5.24[1.62,16.97]$ \\
\hline Liu 2006 & $23 / 39$ & $15 / 36$ & $=$ & $26.17 \%$ & $1.42[0.89,2.26]$ \\
\hline Song 2013 & $66 / 130$ & $15 / 64$ & $\rightarrow$ & $26.04 \%$ & $2.17[1.35,3.48]$ \\
\hline Zhang 2002 & $30 / 36$ & $2 / 28$ & $\longrightarrow$ & $11.85 \%$ & $11.67[3.04,44.71]$ \\
\hline Subtotal $(95 \% \mathrm{Cl})$ & 301 & 223 & & $100 \%$ & $2.82[1.57,5.07]$ \\
\hline \multicolumn{6}{|c|}{ Total events: 157 (Treatment), 43 (Control) } \\
\hline \multicolumn{6}{|c|}{ Heterogeneity: $\mathrm{Tau}^{2}=0.29 ; \mathrm{Chi}^{2}=13.6, \mathrm{df}=4(\mathrm{P}=0.01) ; \mathrm{I}^{2}=70.58 \%$} \\
\hline \multicolumn{6}{|c|}{ Test for overall effect: $Z=3.46(P=0)$} \\
\hline
\end{tabular}

Analysis 1.2. Comparison 1 Intervention versus no intervention/usual care, Outcome 2 Number of incontinent episodes in $\mathbf{2 4}$ hours (mean).

\begin{tabular}{|c|c|c|c|c|c|c|c|}
\hline \multirow[t]{2}{*}{ Study or subgroup } & \multicolumn{2}{|c|}{ Treatment } & \multicolumn{2}{|c|}{ Control } & \multirow{2}{*}{$\begin{array}{l}\text { Mean Difference } \\
\text { Random, } 95 \% \mathrm{Cl}\end{array}$} & \multirow[t]{2}{*}{ Weight } & \multirow{2}{*}{$\begin{array}{l}\text { Mean Difference } \\
\text { Random, } 95 \% \mathrm{Cl}\end{array}$} \\
\hline & $\mathbf{N}$ & Mean(SD) & $\mathbf{N}$ & Mean(SD) & & & \\
\hline \multicolumn{8}{|c|}{ 1.2.1 Behavioural interventions } \\
\hline Tibaek 2005 & 10 & $0.2(0.3)$ & 8 & $1.2(2.5)$ & + & $100 \%$ & $-1[-2.74,0.74]$ \\
\hline 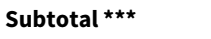 & 10 & & 8 & & $\uparrow$ & $100 \%$ & $-1[-2.74,0.74]$ \\
\hline \multicolumn{8}{|c|}{ Heterogeneity: Not applicable } \\
\hline \multicolumn{8}{|c|}{ Test for overall effect: $Z=1.12(P=0.26)$} \\
\hline \multicolumn{8}{|c|}{ 1.2.2 Physical therapy interventions } \\
\hline Guo 2014 & 32 & $1.6(0.3)$ & 29 & $3.9(0.7)$ & -1] & $35.77 \%$ & $-2.25[-2.54,-1.96]$ \\
\hline Liu 2016a & 27 & $2.1(0.8)$ & 14 & $9.5(3.5)$ & $=$ & $32.27 \%$ & $-7.44[-9.3,-5.58]$ \\
\hline Liu 2016b & 27 & $4.7(1.1)$ & 13 & $9.5(3.5)$ & \# & $31.96 \%$ & $-4.85[-6.8,-2.9]$ \\
\hline Subtotal $\star \star \star$ & 86 & & 56 & & $\checkmark$ & $100 \%$ & $-4.76[-8.1,-1.41]$ \\
\hline \multicolumn{8}{|c|}{ Heterogeneity: $\mathrm{Tau}^{2}=8.1 ; \mathrm{Chi}^{2}=35.2, \mathrm{df}=2(\mathrm{P}<0.0001) ; \mathrm{I}^{2}=94.32 \%$} \\
\hline
\end{tabular}


Analysis 1.3. Comparison 1 Intervention versus no intervention/usual care, Outcome 3 Number of participants cured of all four urinary symptoms.

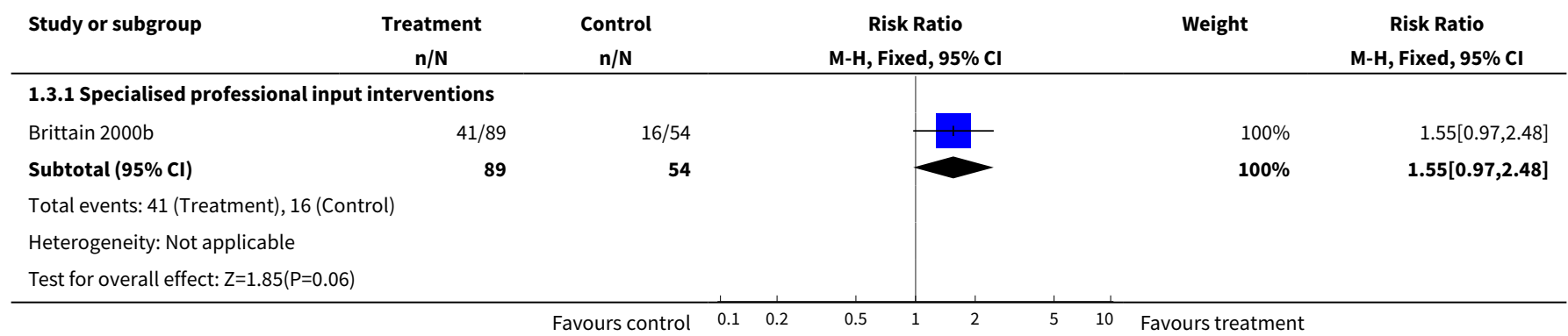

Analysis 1.4. Comparison 1 Intervention versus no intervention/usual care, Outcome 4 Urinary symptoms - frequency (continuous variables).

\begin{tabular}{|c|c|c|c|c|c|c|c|}
\hline \multirow[t]{2}{*}{ Study or subgroup } & \multicolumn{2}{|c|}{ Treatment } & \multicolumn{2}{|c|}{ Control } & \multirow{2}{*}{$\begin{array}{l}\text { Mean Difference } \\
\text { Random, } 95 \% \mathrm{Cl}\end{array}$} & \multirow[t]{2}{*}{ Weight } & \multirow{2}{*}{$\begin{array}{l}\text { Mean Difference } \\
\text { Random, } 95 \% \mathrm{Cl}\end{array}$} \\
\hline & $\mathbf{N}$ & $\operatorname{Mean}(S D)$ & $\mathbf{N}$ & $\operatorname{Mean}(\mathrm{SD})$ & & & \\
\hline \multicolumn{8}{|c|}{ 1.4.1 Behavioural interventions } \\
\hline Tibaek 2005 & 10 & $5.8(1)$ & 8 & $8.1(2.5)$ & +1 & $50.6 \%$ & $-2.3[-4.14,-0.46]$ \\
\hline Tibaek 2017 & 15 & $5.9(2.5)$ & 15 & $7(2.7)$ & & $49.4 \%$ & $-1.1[-2.96,0.76]$ \\
\hline Subtotal $\star \star \star$ & 25 & & 23 & & & $100 \%$ & $-1.71[-3.02,-0.4]$ \\
\hline \multicolumn{8}{|c|}{ Heterogeneity: $\mathrm{Tau}^{2}=0 ; \mathrm{Chi}^{2}=0.81, \mathrm{df}=1(\mathrm{P}=0.37) ; \mathrm{I}^{2}=0 \%$} \\
\hline \multicolumn{8}{|c|}{ 1.4.2 Complementary therapy interventions } \\
\hline Liu 2006 & 32 & $7(2.1)$ & 30 & $12.6(3.5)$ & & $100 \%$ & $-5.57[-7,-4.14]$ \\
\hline 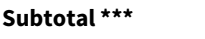 & 32 & & 30 & & & $100 \%$ & $-5.57[-7,-4.14]$ \\
\hline \multicolumn{8}{|c|}{ Heterogeneity: Not applicable } \\
\hline \multicolumn{8}{|c|}{ Test for overall effect: $Z=7.61(P<0.0001)$} \\
\hline \multicolumn{8}{|c|}{ 1.4.3 Physical therapy interventions } \\
\hline Guo 2014 & 32 & $0.7(0.6)$ & 29 & $1.6(0.3)$ & \# & $36.39 \%$ & $-0.9[-1.13,-0.67]$ \\
\hline Liu 2016a & 27 & $7.4(2.9)$ & 14 & $12.8(2.4)$ & & $32.59 \%$ & $-5.43[-7.1,-3.76]$ \\
\hline Liu 2016b & 27 & $10.4(4.1)$ & 13 & $12.8(2.4)$ & 世 & $31.02 \%$ & $-2.37[-4.4,-0.34]$ \\
\hline Subtotal $\star \star \star$ & 86 & & 56 & & & $100 \%$ & $-2.83[-5.75,0.09]$ \\
\hline \multicolumn{8}{|c|}{ Heterogeneity: $\mathrm{Tau}^{2}=6.09 ; \mathrm{Chi}^{2}=29.48, \mathrm{df}=2(\mathrm{P}<0.0001) ; \mathrm{I}^{2}=93.22 \%$} \\
\hline Test for overall effec & & & & & & & \\
\hline
\end{tabular}

Analysis 1.5. Comparison 1 Intervention versus no intervention/usual care, Outcome 5 Urinary symptoms - frequency (dichotomous variables).

\begin{tabular}{|c|c|c|c|c|c|}
\hline Study or subgroup & $\begin{array}{c}\text { Treatment } \\
\mathrm{n} / \mathrm{N} \\
\end{array}$ & $\begin{array}{c}\text { Control } \\
n / N\end{array}$ & $\begin{array}{c}\text { Risk Ratio } \\
\text { M-H, Fixed, } 95 \% \mathrm{Cl}\end{array}$ & Weight & $\begin{array}{c}\text { Risk Ratio } \\
\text { M-H, Fixed, 95\% Cl }\end{array}$ \\
\hline \multicolumn{6}{|c|}{ 1.5.1 Specialised professional input interventions } \\
\hline Brittain 2000b & $98 / 120$ & $59 / 67$ & - & $100 \%$ & $0.93[0.82,1.05]$ \\
\hline Subtotal $(95 \% \mathrm{CI})$ & 120 & 67 & & $100 \%$ & $0.93[0.82,1.05]$ \\
\hline \multicolumn{6}{|c|}{ Total events: 98 (Treatment), 59 (Control) } \\
\hline
\end{tabular}




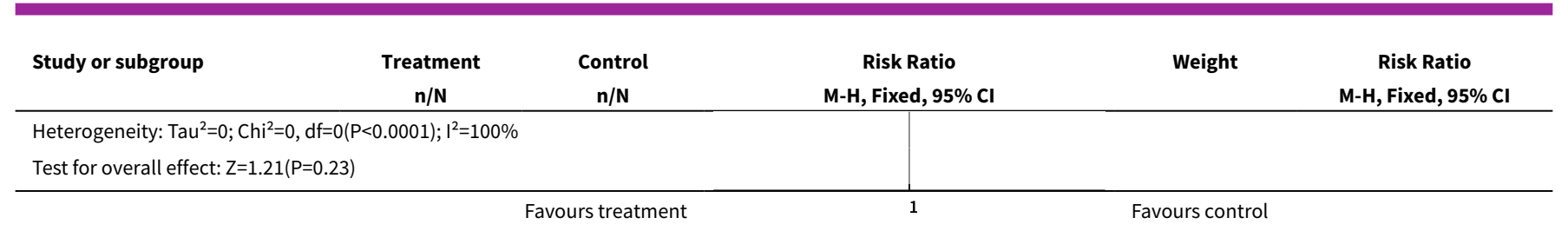

\section{Analysis 1.6. Comparison 1 Intervention versus no intervention/ usual care, Outcome 6 Urinary symptoms - urgency.}

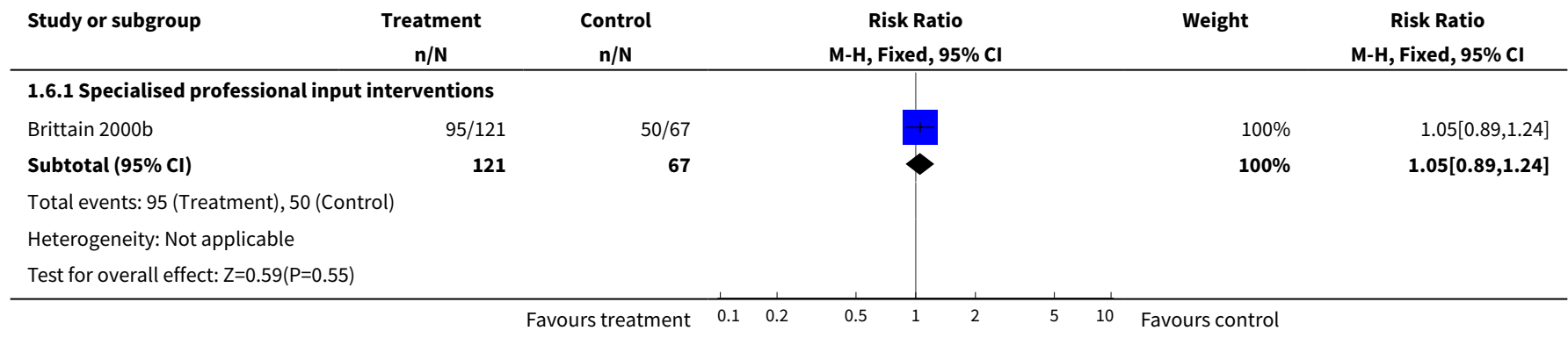

Analysis 1.7. Comparison 1 Intervention versus no intervention/usual care, Outcome 7 Urinary symptoms - nocturia (continuous variables).

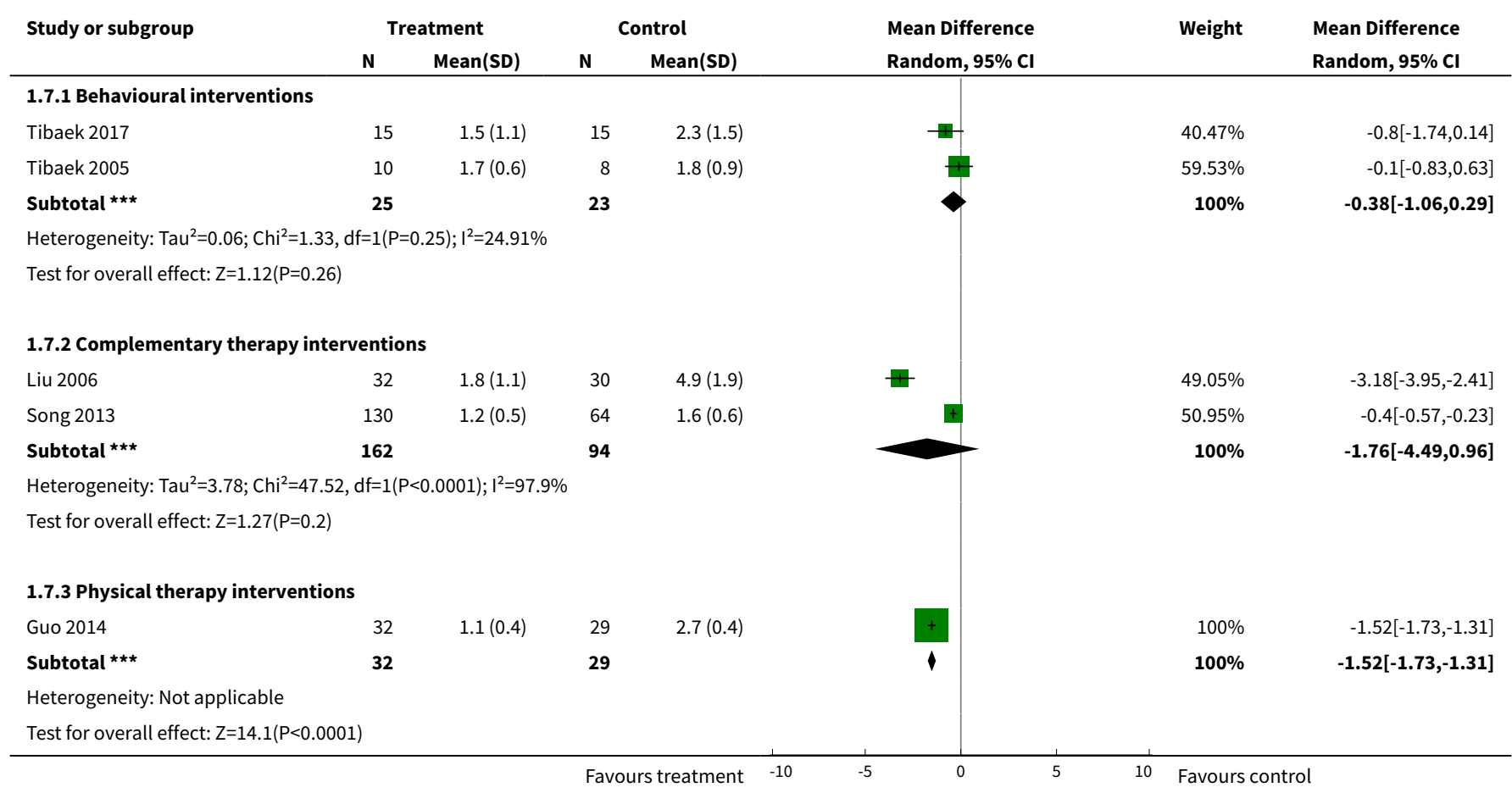


Analysis 1.8. Comparison 1 Intervention versus no intervention/usual care, Outcome 8 Urinary symptoms - nocturia (dichotomous variables).

\begin{tabular}{|c|c|c|c|c|c|}
\hline Study or subgroup & $\begin{array}{c}\text { Treatment } \\
\mathrm{n} / \mathrm{N} \\
\end{array}$ & $\begin{array}{c}\text { Control } \\
\mathrm{n} / \mathrm{N}\end{array}$ & $\begin{array}{c}\text { Risk Ratio } \\
\text { M-H, Fixed, } 95 \% \text { Cl }\end{array}$ & Weight & $\begin{array}{c}\text { Risk Ratio } \\
\text { M-H, Fixed, 95\% Cl }\end{array}$ \\
\hline \multicolumn{6}{|c|}{ 1.8.1 Specialised professional input interventions } \\
\hline Brittain 2000b & $102 / 119$ & $60 / 67$ & - & $100 \%$ & $0.96[0.86,1.07]$ \\
\hline Subtotal $(95 \% \mathrm{Cl})$ & 119 & 67 & & $100 \%$ & $0.96[0.86,1.07]$ \\
\hline \multicolumn{6}{|c|}{ Total events: 102 (Treatment), 60 (Control) } \\
\hline \multicolumn{6}{|c|}{ Heterogeneity: $\mathrm{Tau}^{2}=0 ; \mathrm{Chi}^{2}=0, \mathrm{df}=0(\mathrm{P}<0.0001) ; \mathrm{I}^{2}=100 \%$} \\
\hline
\end{tabular}

Analysis 1.9. Comparison 1 Intervention versus no intervention/usual care, Outcome 9 Physical measures: mean function of the pelvic floor muscle.

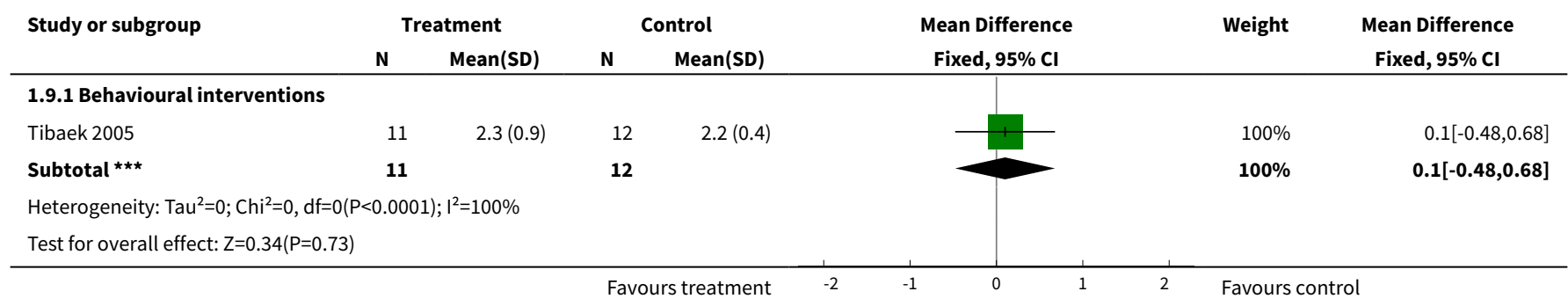

Analysis 1.10. Comparison 1 Intervention versus no intervention/usual care, Outcome 10 Health status and quality of life - health status, mean total score 36-Item Short Form.

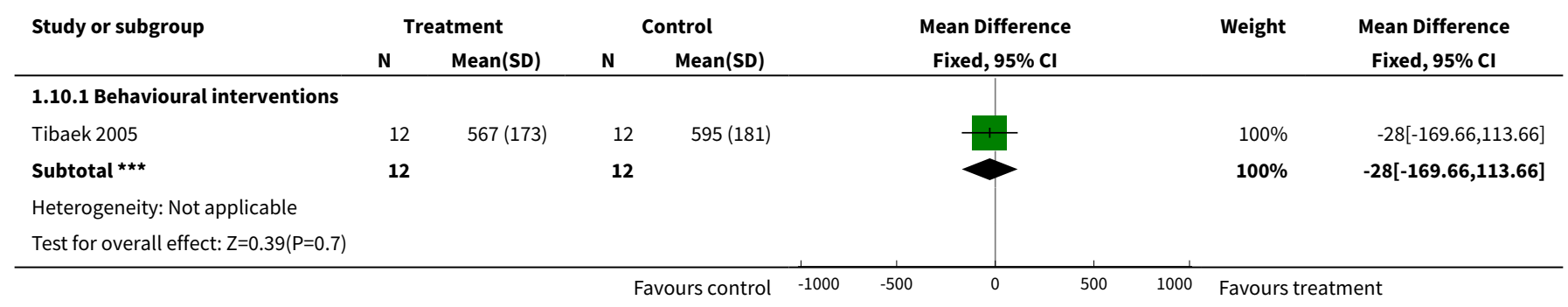

Analysis 1.11. Comparison 1 Intervention versus no intervention/ usual care, Outcome 11 Health status and quality of life - quality of life.

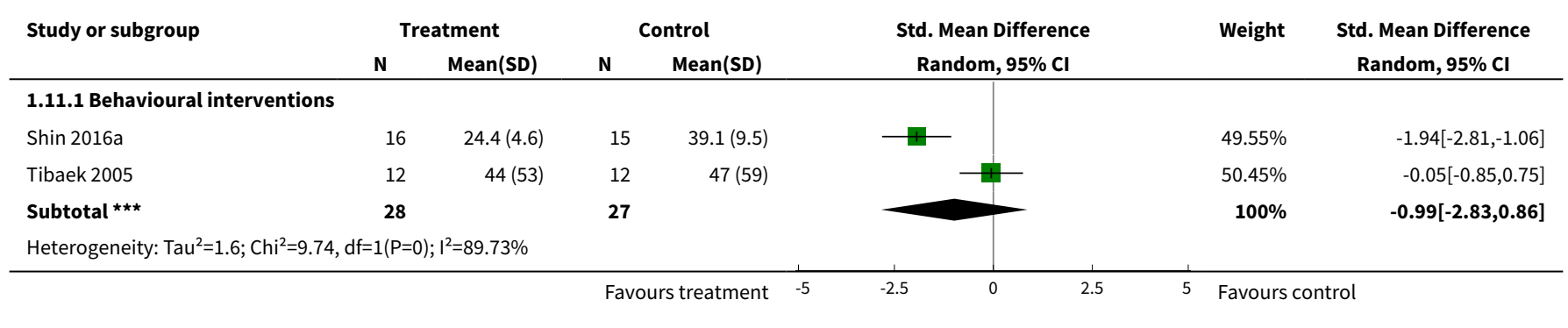




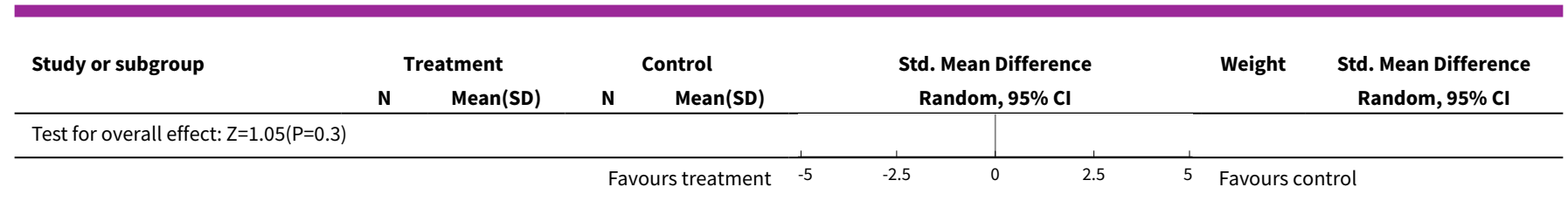

Analysis 1.12. Comparison 1 Intervention versus no intervention/usual care, Outcome 12 Functional ability - mean Barthel score (continuous variables).

\begin{tabular}{|c|c|c|c|c|c|c|c|c|}
\hline \multirow[t]{2}{*}{ Study or subgroup } & \multicolumn{2}{|c|}{ Treatment } & \multicolumn{2}{|c|}{ Control } & \multirow{2}{*}{\multicolumn{2}{|c|}{$\begin{array}{l}\text { Mean Difference } \\
\text { Random, } 95 \% \mathrm{Cl}\end{array}$}} & \multirow[t]{2}{*}{ Weight } & \multirow{2}{*}{$\begin{array}{l}\text { Mean Difference } \\
\text { Random, } 95 \% \mathrm{Cl}\end{array}$} \\
\hline & $\mathbf{N}$ & $\operatorname{Mean}(S D)$ & $\mathbf{N}$ & Mean(SD) & & & & \\
\hline \multicolumn{9}{|c|}{ 1.12.1 Physical therapy interventions } \\
\hline Liu 2016a & 27 & $65.8(16.6)$ & 14 & $52.5(8.3)$ & & $\rightarrow$ & $45.22 \%$ & $13.3[5.66,20.94]$ \\
\hline Liu 2016b & 27 & $57.9(10.3)$ & 13 & $52.5(8.3)$ & & $\rightarrow$ & $54.78 \%$ & $5.4[-0.55,11.35]$ \\
\hline Subtotal $\star \star \star$ & 54 & & 27 & & & & $100 \%$ & $8.97[1.27,16.68]$ \\
\hline \multicolumn{9}{|c|}{ Heterogeneity: $\mathrm{Tau}^{2}=19.01 ; \mathrm{Chi}^{2}=2.56, \mathrm{df}=1(\mathrm{P}=0.11) ; \mathrm{I}^{2}=60.93 \%$} \\
\hline \multicolumn{9}{|c|}{ Test for overall effect: $\mathrm{Z}=2.28(\mathrm{P}=0.02)$} \\
\hline
\end{tabular}

Analysis 1.13. Comparison 1 Intervention versus no intervention/usual care, Outcome 13 Participant satisfaction - numbers who were dissatisfied.

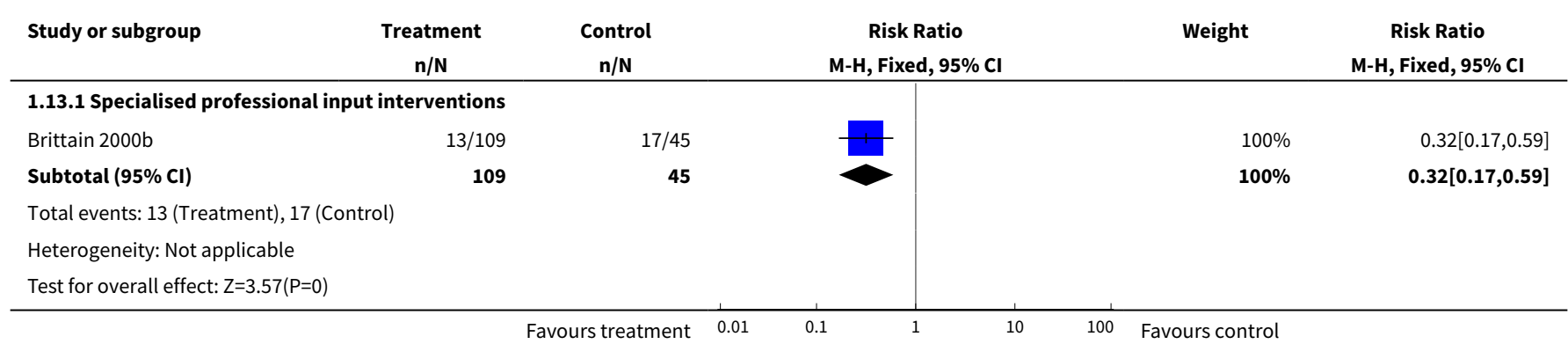

\section{Comparison 2. Intervention versus placebo}

\begin{tabular}{lllll}
\hline Outcome or subgroup title & No. of studies & $\begin{array}{l}\text { No. of partici- } \\
\text { pants }\end{array}$ & Statistical method & Effect size \\
\hline $\begin{array}{l}1 \text { Number of participants continent af- } \\
\text { ter treatment }\end{array}$ & 1 & 54 & $\begin{array}{l}\text { Risk Ratio (M-H, Fixed, 95\% } \\
\text { Cl) }\end{array}$ & $0.75[0.19,3.04]$ \\
\hline $\begin{array}{l}2 \text { Number of incontinent episodes - } \\
\text { mean per day }\end{array}$ & 1 & 39 & $\begin{array}{l}\text { Mean Difference (IV, Fixed, } \\
95 \% \text { Cl) }\end{array}$ & $-1.10[-3.99,1.79]$ \\
\hline $\begin{array}{l}3 \text { Number of incontinent episodes - } \\
\text { mean per week }\end{array}$ & 1 & & $\begin{array}{l}\text { Paired samples mean (Ran- } \\
\text { dom, 95\% Cl) }\end{array}$ & $-3.88[-8.47,0.70]$ \\
\hline $\begin{array}{l}\text { 3.1 Oestrogen vs placebo (mild inconti- } \\
\text { nence) }\end{array}$ & 1 & $\begin{array}{l}\text { Paired samples mean (Ran- } \\
\text { dom, 95\% Cl) }\end{array}$ & $-1.71[-3.51,0.09]$ \\
\hline
\end{tabular}




\begin{tabular}{lllll}
\hline Outcome or subgroup title & No. of studies & $\begin{array}{l}\text { No. of partici- } \\
\text { pants }\end{array}$ & Statistical method & Effect size \\
\hline $\begin{array}{l}\text { 3.2 Oestrogen vs placebo (severe in- } \\
\text { continence) }\end{array}$ & 1 & & $\begin{array}{l}\text { Paired samples mean (Ran- } \\
\text { dom, 95\% Cl) }\end{array}$ & $-6.4[-9.47,-3.33]$ \\
\hline $\begin{array}{l}4 \text { Health status and quality of life - } \\
\text { quality of life }\end{array}$ & 1 & 30 & $\begin{array}{l}\text { Mean Difference (IV, Fixed, } \\
95 \% \text { Cl) }\end{array}$ & $3.90[-4.25,12.05]$ \\
\hline
\end{tabular}

Analysis 2.1. Comparison 2 Intervention versus placebo, Outcome 1 Number of participants continent after treatment.

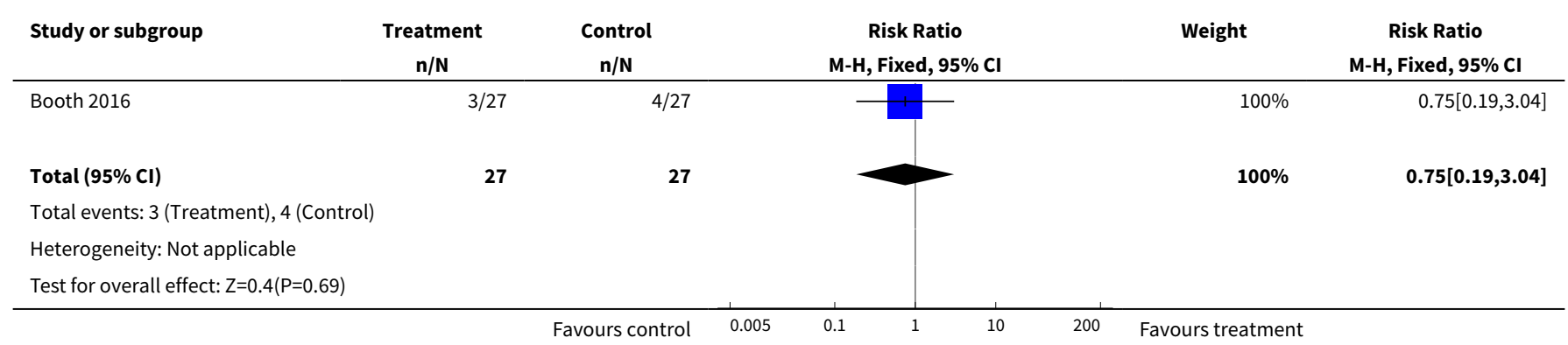

Analysis 2.2. Comparison 2 Intervention versus placebo, Outcome 2 Number of incontinent episodes - mean per day.

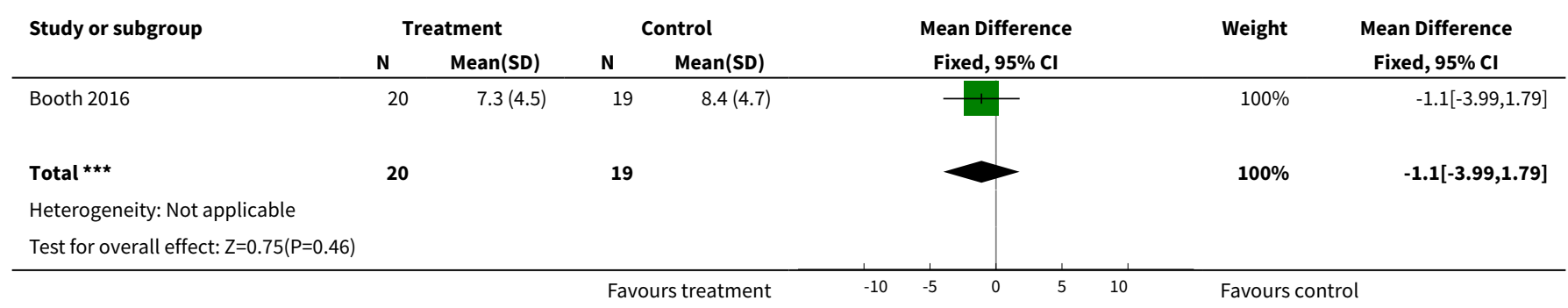

Analysis 2.3. Comparison 2 Intervention versus placebo, Outcome 3 Number of incontinent episodes - mean per week.

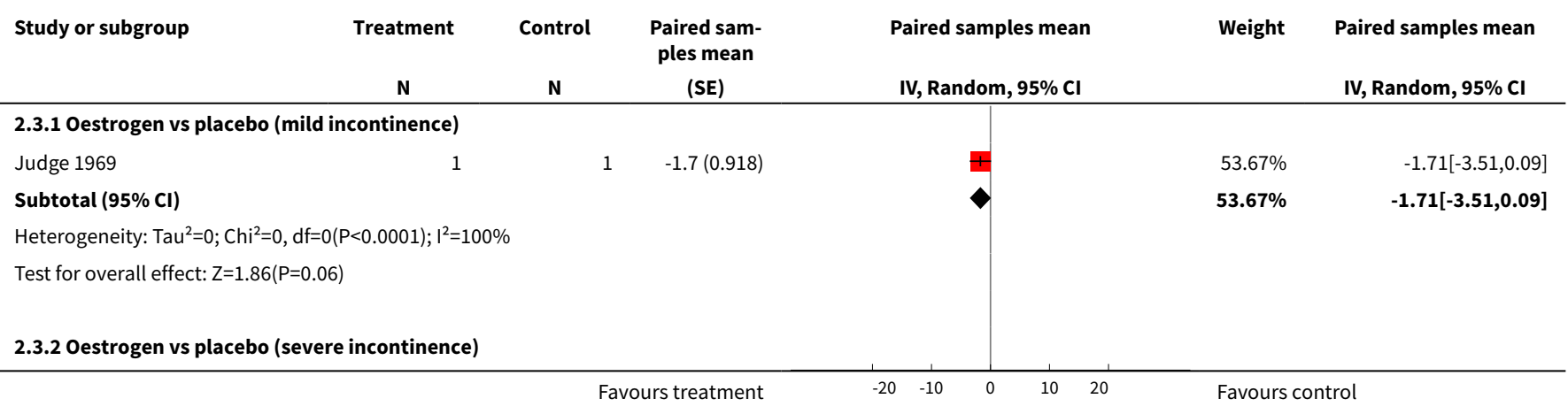




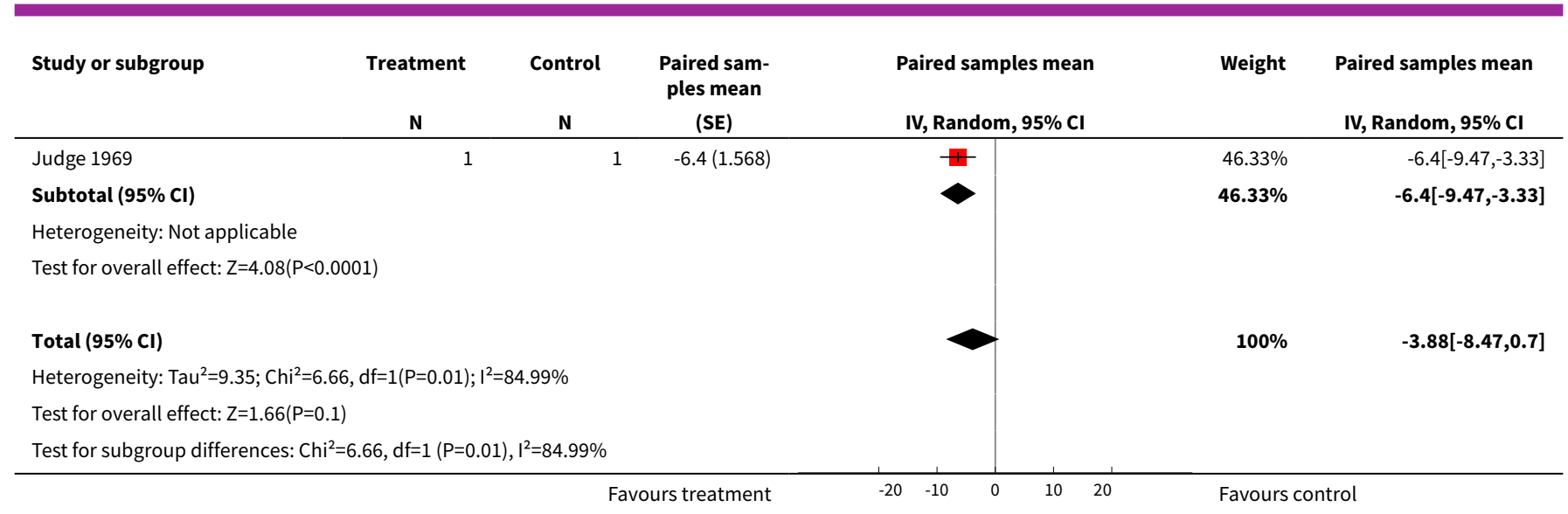

Analysis 2.4. Comparison 2 Intervention versus placebo, Outcome 4 Health status and quality of life - quality of life.

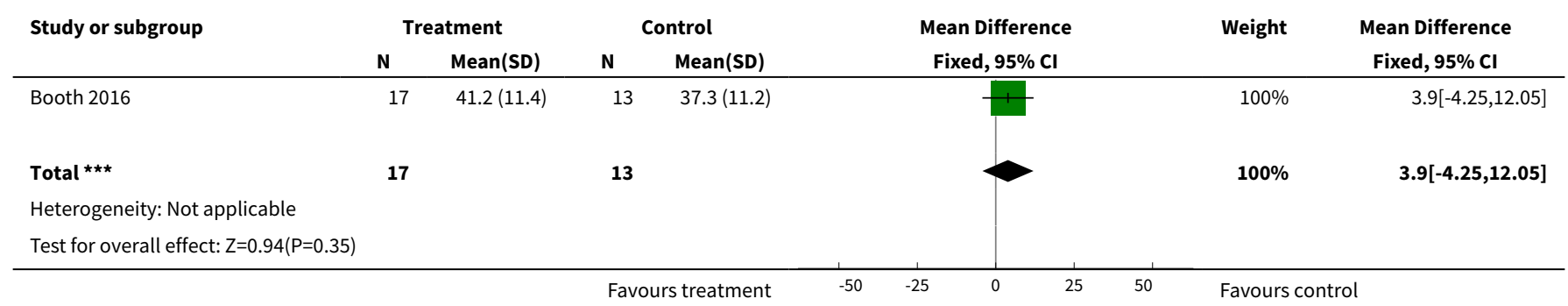

\section{Comparison 4. Combined intervention versus single intervention}

\begin{tabular}{|c|c|c|c|c|}
\hline Outcome or subgroup title & No. of studies & $\begin{array}{l}\text { No. of partici- } \\
\text { pants }\end{array}$ & Statistical method & Effect size \\
\hline $\begin{array}{l}1 \text { Number of participants continent after } \\
\text { treatment }\end{array}$ & 1 & & $\begin{array}{l}\text { Risk Ratio (M-H, Fixed, } \\
95 \% \mathrm{Cl})\end{array}$ & Subtotals only \\
\hline $\begin{array}{l}1.1 \text { Sensory-motor biofeedback device + } \\
\text { timed voiding vs timed voiding alone }\end{array}$ & 1 & 23 & $\begin{array}{l}\text { Risk Ratio (M-H, Fixed, } \\
95 \% \mathrm{Cl})\end{array}$ & $0.55[0.06,5.21]$ \\
\hline 2 Number of incontinent episodes - mean & 1 & & $\begin{array}{l}\text { Mean Difference (IV, Fixed, } \\
95 \% \mathrm{CI})\end{array}$ & Subtotals only \\
\hline $\begin{array}{l}\text { 2.1 Sensory-motor biofeedback device + } \\
\text { timed voiding vs timed voiding alone }\end{array}$ & 1 & 23 & $\begin{array}{l}\text { Mean Difference (IV, Fixed, } \\
95 \% \mathrm{CI})\end{array}$ & $2.2[0.12,4.28]$ \\
\hline
\end{tabular}


Analysis 4.1. Comparison 4 Combined intervention versus single intervention, Outcome 1 Number of participants continent after treatment.

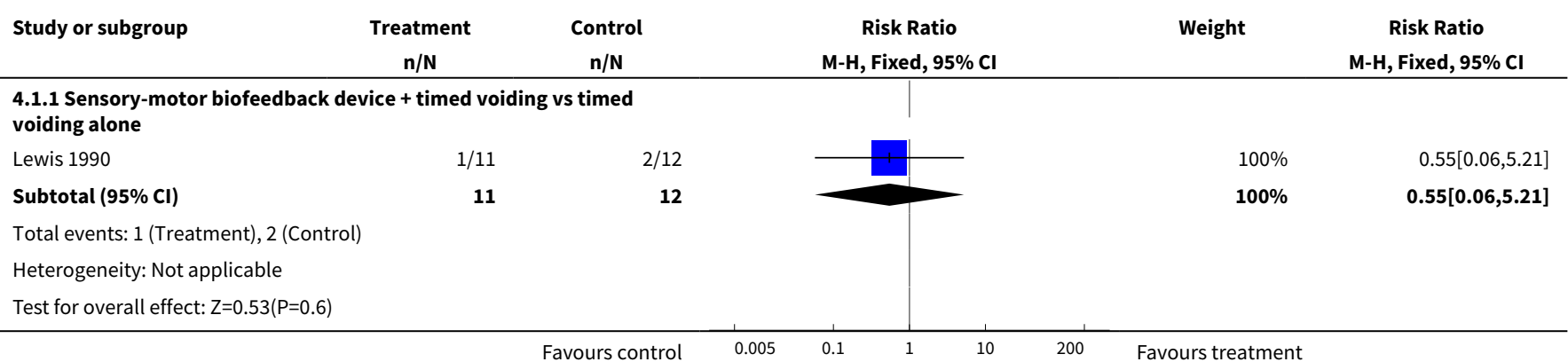

Analysis 4.2. Comparison 4 Combined intervention versus single intervention, Outcome 2 Number of incontinent episodes - mean.

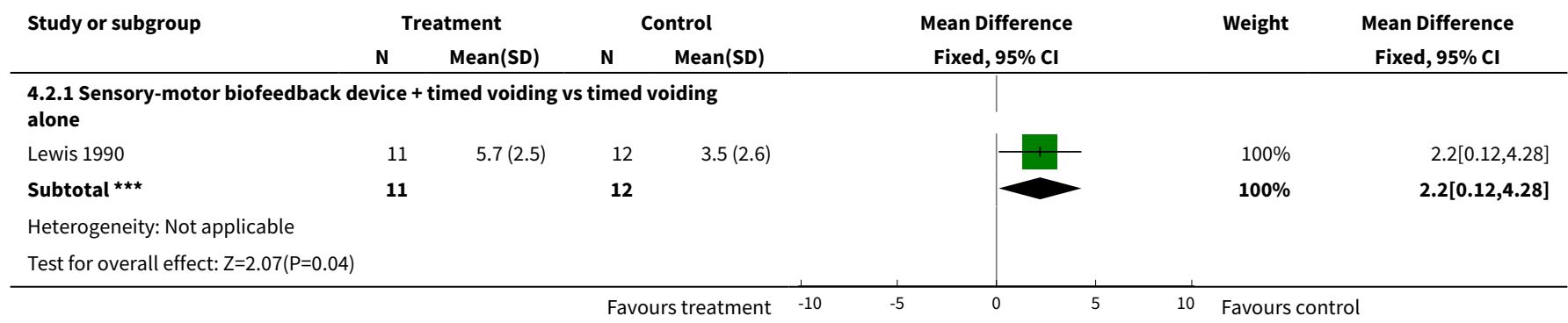

\section{Comparison 5. Specific intervention versus attention control}

\begin{tabular}{lllll}
\hline Outcome or subgroup title & No. of studies & $\begin{array}{l}\text { No. of partici- } \\
\text { pants }\end{array}$ & Statistical method & Effect size \\
\hline $\begin{array}{l}1 \text { Number of participants continent af- } \\
\text { ter treatment }\end{array}$ & 1 & & $\begin{array}{l}\text { Risk Ratio (M-H, Fixed, 95\% } \\
\mathrm{Cl})\end{array}$ & Subtotals only \\
\hline $\begin{array}{l}1.1 \text { TPTNS versus stretching exercises } \\
\text { n }\end{array}$ & 1 & 24 & $\begin{array}{l}\text { Risk Ratio (M-H, Fixed, 95\% } \\
\text { Cl) }\end{array}$ & $1.33[0.38,4.72]$ \\
\hline
\end{tabular}

Analysis 5.1. Comparison 5 Specific intervention versus attention control, Outcome 1 Number of participants continent after treatment.

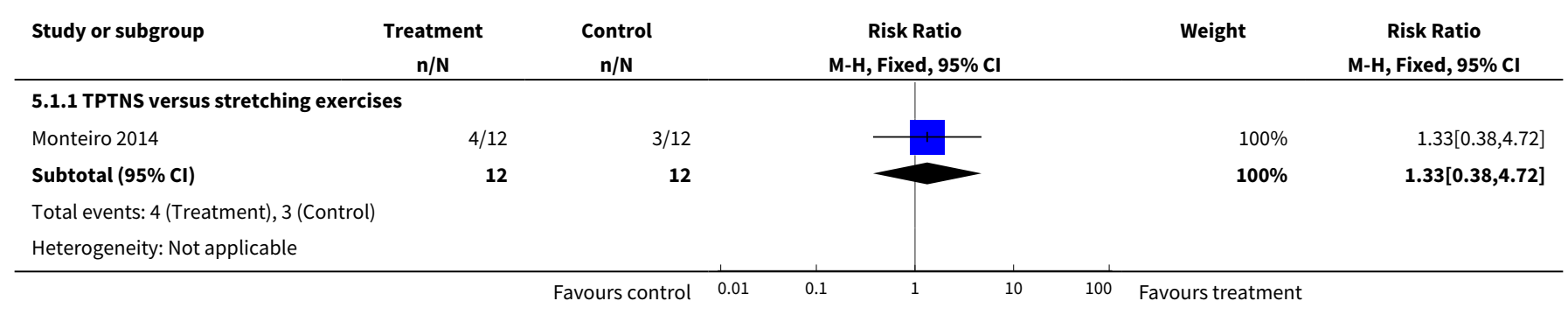




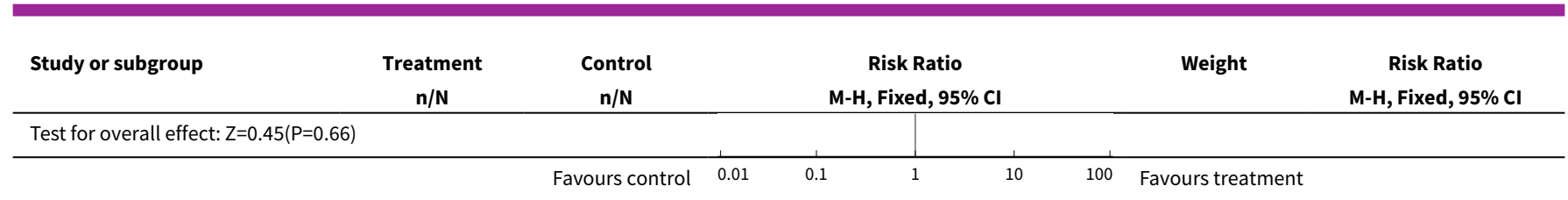

\section{ADDITIONAL TABLES}

Table 1. Continence status prior to stroke, diagnostic criteria for incontinence and description of incontinence by study authors

\begin{tabular}{|c|c|c|c|}
\hline Study IDs & $\begin{array}{l}\text { Continent prior to } \\
\text { stroke }\end{array}$ & $\begin{array}{l}\text { Diagnostic criteria for assess- } \\
\text { ment of incontinence as re- } \\
\text { ported by study authors }\end{array}$ & $\begin{array}{l}\text { Description of participant incontinence by study } \\
\text { authors }\end{array}$ \\
\hline Booth 2016 & Yes & Not reported & At least once weekly \\
\hline Brittain 2000b & Unclear & $\begin{array}{l}\text { Yes (structured 1-hour assess- } \\
\text { ment by continence nurse prac- } \\
\text { titioner) }\end{array}$ & $\begin{array}{l}\text { Self-reported clinical symptoms of leakage sever- } \\
\text { al times per month or more, frequency every } \geq 30 \\
\text { minutes, nocturia, urgency }\end{array}$ \\
\hline Chu 1997 & Unclear & Not reported & Not reported \\
\hline Chu 2011 & Yes & $\begin{array}{l}\text { Yes (according to "neurological } \\
\text { disease syndrome") }\end{array}$ & $\begin{array}{l}\text { According to "neurological disease syndrome" neu- } \\
\text { rogenic bladder with incontinence }\end{array}$ \\
\hline Gelber 1997a & Unclear & Not reported & Normal urodynamic studies with incontinence \\
\hline Gelber 1997b & Unclear & Not reported & Bladder hyper-reflexia \\
\hline Guo 2014 & Yes & Yes (OABSS) & Post-stroke urinary incontinence \\
\hline Judge 1969 & Unclear & Not reported & Mild or severe incontinence \\
\hline Lewis 1990 & Unclear & Not reported & Post-stroke urge urinary incontinence \\
\hline Liu 2006 & Yes & $\begin{array}{l}\text { Yes (Barthel Index - continence } \\
\text { item) }\end{array}$ & $\begin{array}{l}\text { Completely incontinent, partially incontinent and } \\
\text { self-controlling }\end{array}$ \\
\hline Liu 2013 & Yes & Yes (urodynamic assessment) & Post-stroke detrusor overactivity \\
\hline Liu 2016a & Yes & Yes (OABSS) & Post-stroke urinary incontinence \\
\hline Liu 2016b & Yes & Yes (OABSS) & Post-stroke urinary incontinence \\
\hline Monteiro 2014 & Yes & $\begin{array}{l}\text { Yes (Barthel Index - Bladder } \\
\text { item) }\end{array}$ & Post-stroke neurogenic overactive bladder \\
\hline Shin $2016 a$ & Yes & $\begin{array}{l}\text { Yes (Bristol Female Urinary } \\
\text { Symptoms Questionnaire) }\end{array}$ & Post-stroke stress urinary incontinence \\
\hline Song 2013 & Yes & Not reported & Post-stroke urinary incontinence \\
\hline Tibaek 2005 & Yes & Yes (ICS definition) & Post-stroke urinary incontinence \\
\hline
\end{tabular}


Table 1. Continence status prior to stroke, diagnostic criteria for incontinence and description of incontinence by study authors (Continued)

\begin{tabular}{llll} 
Tibaek 2017 & Yes & Yes (ICS definition) & Lower urinary tract symptoms \\
\hline Zhang 1996 & Unclear & Not reported & Post-stroke urinary incontinence \\
\hline Zhang 2002 & Unclear & Not reported & Post-stroke urinary incontinence \\
\hline Zhou 1999 & Unclear & Not reported & \\
\hline
\end{tabular}

ICS: International Continence Society; OABSS: Overactive Bladder Symptom Score.

Table 2. Stroke history, stroke diagnostic information and stroke description

\begin{tabular}{|c|c|c|c|}
\hline Study IDs & $\begin{array}{l}\text { Stroke history of } \\
\text { participants (any } \\
\text { previous stroke) }\end{array}$ & $\begin{array}{l}\text { Stroke diagnostic information provided } \\
\text { by study authors }\end{array}$ & $\begin{array}{l}\text { Stroke description of study partici- } \\
\text { pants }\end{array}$ \\
\hline Booth 2016 & Not reported & According to WHO 1989 criteria & $\begin{array}{l}\text { Ischaemic }(87.8 \%) \\
\text { Haemorrhagic }(10.2 \%) \\
\text { Other }(2 \%)\end{array}$ \\
\hline Brittain 2000b & Not reported & Postal screening questionnaire - self-report & Not reported \\
\hline Chu 1997 & Not reported & Not reported & Multi-focal cerebral infarction \\
\hline Chu 2011 & $\begin{array}{l}\text { First or repeated } \\
\text { episode of stroke }\end{array}$ & $\begin{array}{l}\text { According to the Fourth National Confer- } \\
\text { ence on Cerebrovascular Disease }\end{array}$ & $\begin{array}{l}\text { Ischaemic (73\%) } \\
\text { Haemorrhagic (27\%) }\end{array}$ \\
\hline Gelber 1997a & Not reported & Not reported & Unilateral stroke \\
\hline Gelber 1997b & Not reported & Not reported & Unilateral stroke \\
\hline Guo 2014 & Not reported & Not reported & $\begin{array}{l}\text { Ischaemic (77\%) } \\
\text { Haemorrhagic (23\%) }\end{array}$ \\
\hline Judge 1969 & Not reported & Not reported & Cerebrovascular accidents \\
\hline Lewis 1990 & Not reported & Not reported & $\begin{array}{l}\text { Ischaemic }(78.2 \%) \\
\text { Haemorrhagic }(21.8 \%)\end{array}$ \\
\hline Liu 2006 & Not reported & Not reported & $\begin{array}{l}\text { Ischaemic (80\%) } \\
\text { Haemorrhagic (20\%) }\end{array}$ \\
\hline Liu 2013 & Not reported & Confirmation by CT or MRI scan & $\begin{array}{l}\text { Ischaemic }(45.5 \%) \\
\text { Haemorrhagic }(54.5 \%)\end{array}$ \\
\hline Liu 2016a & Not reported & Not reported & $\begin{array}{l}\text { Ischaemic }(72.8 \%) \\
\text { Haemorrhagic }(27.2 \%)\end{array}$ \\
\hline Liu 2016b & Not reported & Not reported & Ischaemic (72.8\%) \\
\hline
\end{tabular}


Table 2. Stroke history, stroke diagnostic information and stroke description (Continued)

Haemorrhagic (27.2\%)

\begin{tabular}{lll}
\hline Monteiro $2014 \quad$ Not reported & $\begin{array}{l}\text { Clinical and neuroimaging evaluation ac- } \\
\text { cording to established criteria of Brazilian }\end{array} \quad$ Ischaemic (100\%)
\end{tabular}

Cerebrovascular Disease Society

\begin{tabular}{llll}
\hline Shin 2016a & Not reported & Not reported & Not reported \\
\hline Song 2013 & Not reported & $\begin{array}{l}\text { According 1995 National Meeting on Cere- } \\
\text { brovascular Accidents }\end{array}$ & Ischaemic (49.5\%) \\
\hline Tibaek 2005 & First ever stroke & According to WHO 1989 criteria & Ischaemic (100\%) \\
\hline Tibaek 2017 & $\begin{array}{l}\text { 16.7\% had 1 previ- } \\
\text { ous stroke }\end{array}$ & According to WHO 1989 criteria & Not reported \\
\hline Zhang 1996 & Not reported & Confirmation by CT or MRI scan & Ischaemic and \\
\hline Zhang 2002 & Not reported & Not reported & Haemorrhagic, figures not reported. \\
\hline Zhou 1999 & Not reported & $\begin{array}{l}\text { According to criteria from Chinese Diagnos- } \\
\text { tic Guidelines }\end{array}$ & Ischaemic (66.3\%) \\
\hline
\end{tabular}

CT: computed tomography; MRI: magnetic resonance imaging.

Table 3. Primary and secondary outcomes as described by study authors

\begin{tabular}{|c|c|c|c|c|}
\hline Study IDs & Primary outcome(s) & Measured by & Secondary outcome(s) & Measured by \\
\hline \multirow[t]{8}{*}{ Booth 2016} & Number of incontinent episodes & ICIQ-UI-SF & Severity & ICIQ-UI-SF \\
\hline & & & Urinary symptoms & AUASI* \\
\hline & & & $\begin{array}{l}\text { Urgency perception } \\
\text { scores }\end{array}$ & Bladder diary \\
\hline & & & $\begin{array}{l}\text { Postvoid residual vol- } \\
\text { ume }\end{array}$ & Bladder scan \\
\hline & & & Quality of life & EQ-5D-5L \\
\hline & & & & ICIQLUTSqol \\
\hline & & & Adverse events & Participant reports \\
\hline & & & $A D L$ & Barthel Index \\
\hline Brittain 2000b & Number of people incontinent & $\begin{array}{l}\text { Nurse assess- } \\
\text { ment and self-re- } \\
\text { port }\end{array}$ & $\begin{array}{l}\text { Urinary symptoms } \\
\text { Satisfaction with ser- } \\
\text { vice }\end{array}$ & $\begin{array}{l}\text { Nurse assessment and } \\
\text { self-report }\end{array}$ \\
\hline Chu 1997 & Number regaining continence & Not reported & None reported & - \\
\hline
\end{tabular}


Table 3. Primary and secondary outcomes as described by study authors (Continued)

\begin{tabular}{|c|c|c|c|c|}
\hline \multirow[t]{3}{*}{ Chu 2011} & \multirow[t]{3}{*}{ Number of people incontinent } & \multirow[t]{3}{*}{$\begin{array}{l}\text { Urinary Conti- } \\
\text { nence Status } \\
\text { Grading ( } 4 \text { cate- } \\
\text { gories) }\end{array}$} & Severity & $\begin{array}{l}\text { Urinary Continence } \\
\text { Status Grading ( } 4 \text { cate- } \\
\text { gories) } \\
\text { Participant reports }\end{array}$ \\
\hline & & & Urinary symptoms & $\begin{array}{l}\text { Clinical aggregate } \\
\text { score }\end{array}$ \\
\hline & & & Adverse events & Not reported \\
\hline Gelber 1997a & $\begin{array}{l}\text { Number of incontinent episodes per } \\
\text { day for each month of treatment for } 1 \\
\text { year }\end{array}$ & Not reported & None reported & - \\
\hline Gelber 1997b & $\begin{array}{l}\text { Number of incontinent episodes per } \\
\text { day for each month of treatment for } 1 \\
\text { year }\end{array}$ & Not reported & None reported & - \\
\hline \multirow[t]{2}{*}{ Guo 2014} & Primary outcome not stated & - & Urinary symptoms & OABSS \\
\hline & & & $A D L$ & Barthel Index \\
\hline Judge 1969 & $\begin{array}{l}\text { Number of incontinent episodes per } \\
\text { week }\end{array}$ & Not reported & None reported & - \\
\hline Lewis 1990 & Number of incontinent episodes & Not reported & - & - \\
\hline Liu 2006 & $\begin{array}{l}\text { Number of incontinent episodes day- } \\
\text { time and night-time, urinary symp- } \\
\text { toms }\end{array}$ & $\begin{array}{l}\text { Barthel Index - } \\
\text { continence item }\end{array}$ & - & - \\
\hline Liu 2013 & $\begin{array}{l}\text { Maximum cystometric capacity, blad- } \\
\text { der compliance, detrusor leak point } \\
\text { pressure. Number of incontinence } \\
\text { episodes per day, urinary symptoms }\end{array}$ & $\begin{array}{l}\text { Urodynamic as- } \\
\text { sessment ac- } \\
\text { cording to AUA/ } \\
\text { SUFU guidelines }\end{array}$ & None reported & - \\
\hline \multirow[t]{2}{*}{ Liu 2016a } & Primary outcome not stated & - & $\begin{array}{l}\text { Number of incontinent } \\
\text { episodes, urodynamic } \\
\text { assessment, voiding di- } \\
\text { ary }\end{array}$ & OABSS \\
\hline & & & $A D L$ & Barthel Index \\
\hline \multirow[t]{2}{*}{ Liu 2016b } & Primary outcome not stated & - & $\begin{array}{l}\text { Number of incontinent } \\
\text { episodes, urodynamic } \\
\text { assessment, voiding di- } \\
\text { ary }\end{array}$ & OABSS \\
\hline & & & $A D L$ & Barthel Index \\
\hline Monteiro 2014 & Primary outcome not stated & - & Urinary symptoms & $\begin{array}{l}\text { Barthel Index (Bladder } \\
\text { item) }\end{array}$ \\
\hline Shin 2016a & Primary outcome not stated & - & $\begin{array}{l}\text { Contractility and mus- } \\
\text { cle activity of the pelvic } \\
\text { floor muscle }\end{array}$ & $\begin{array}{l}\text { Perineometer and } \\
\text { pelvic floor elec- } \\
\text { tromyography }\end{array}$ \\
\hline
\end{tabular}


Table 3. Primary and secondary outcomes as described by study authors (Continued)

Urinary symptoms Bristol Female Lower Urinary Tract Symptoms Questionnaire

\begin{tabular}{|c|c|c|c|c|}
\hline \multirow[t]{2}{*}{ Song 2013} & \multirow[t]{2}{*}{ Primary outcome not stated } & \multirow[t]{2}{*}{-} & Urinary symptoms & $\begin{array}{l}\text { Scoring of urinary } \\
\text { symptoms control } \\
\text { score }(0=\text { totally con- } \\
\text { trolled, } 1=\text { partial con- } \\
\text { trol, } 2 \text { = fully no con- } \\
\text { trol) }\end{array}$ \\
\hline & & & Postvoid residual urine & Bladder scan \\
\hline \multirow[t]{2}{*}{ Tibaek 2005} & $\begin{array}{l}\text { Number of incontinent episodes, num- } \\
\text { ber of pads used, frequency }\end{array}$ & $\begin{array}{l}\text { 3-day voiding di- } \\
\text { ary }\end{array}$ & $\begin{array}{l}\text { Vaginal palpation of } \\
\text { PFM }\end{array}$ & Physical examination \\
\hline & 24-hour home pad test & & & \\
\hline \multirow[t]{3}{*}{ Tibaek 2017} & $\begin{array}{l}\text { Urinary symptoms, frequency and } \\
\text { severity }\end{array}$ & $\begin{array}{l}\text { DAN-PSS-1 ques- } \\
\text { tionnaire }\end{array}$ & $\begin{array}{l}\text { Digital anal palpation of } \\
\text { PFM }\end{array}$ & Physical examination \\
\hline & $\begin{array}{l}\text { Frequency, number of incontinence } \\
\text { episodes, number of pads used }\end{array}$ & $\begin{array}{l}\text { 3-day voiding di- } \\
\text { ary }\end{array}$ & Health status & SF-36 \\
\hline & 24-hour pad test & Pad test & & \\
\hline Zhang 1996 & $\begin{array}{l}\text { Number of people with urinary inconti- } \\
\text { nence }\end{array}$ & Not reported & - & - \\
\hline Zhang 2002 & $\begin{array}{l}\text { Number of people with urinary inconti- } \\
\text { nence }\end{array}$ & Not reported & - & - \\
\hline Zhou 1999 & $\begin{array}{l}\text { Number of people with urinary inconti- } \\
\text { nence }\end{array}$ & Not reported & - & - \\
\hline
\end{tabular}

ADL: activities of daily living; AUASI: American Urological Association Symptom Index; AUA/SUFU: American Urological Association/ Society of Urodynamics, Female Pelvic Medicine and Urogenital Reconstruction guidelines; DAN-PSS-1: Danish Prostatic Symptom Score; EQ-5D-5L: EuroQol Five-Dimensional Questionnaire; ICIQLUTSqol: International Consultation on Incontinence Questionnaire - Lower Urinary Tract Symptoms Quality of Life; ICIQ-UI-SF: International Consultation on Incontinence Questionnaire-Urinary Incontinence-Short Form; OABSS: Overactive Bladder Symptom Score; PFM: pelvic floor muscle; SF-36: 36-Item Short Form Survey.

\section{APPENDICES}

\section{Appendix 1. Search terms used to search the Cochrane Incontinence and Cochrane Stroke Specialised Registers}

Both the Cochrane Incontinence and Cochrane Stroke Specialised Registers were searched for this review update. The dates of the most recent searches of the Specialised Registers for this review update were: 30 October 2017 (Cochrane Stroke Register) and 1 November 2017 (Cochrane Incontinence Register).

\section{Cochrane Incontinence Specialised Register}

The terms used to search the Cochrane Incontinence Specialised Register are given below:

((\{Topic.urine.incon.Stroke.\} OR \{TOPIC.URINE.INCON.UNKNOWN.STROKE.\} OR \{TOPIC.URINE.INCON.NEUROGENIC.\} (in keywords field)) OR

$\left(\left\{\right.\right.$ stroke $\left.\backslash^{\star}\right\}$ OR $\left.\{\text { cerebrovascular }\rangle^{\star}\right\}$ (in title1 field))

OR

(\{TOPIC.URINE.NEUROGENIC.\} OR \{TOPIC.URINE.NEUROGENIC.stroke.\} (in keywords field))) 
AND

(\{DESIGN.CCT $\left.{ }^{\star}\right\}$ OR \{DESIGN.RCT* (in keywords field))

(All searches were in Reference Manager 2012).

\section{Cochrane Stroke Specialised Register}

Each study in the Cochrane Stroke Specialised Register is coded to reflect the stage of the condition at which the intervention is given, the type of condition being treated, and the type of intervention and control group.

To search the Cochrane Stroke Group register the following search terms (codes) were used:

Search method: 1

Stage: Not specified

Disease: Not specified

Condition: Incontinence (Urine/Faecal)

Intervention type: Not specified

Intervention code: Not specified

Search method: 2

Stage: Not specified

Disease: Not specified

Condition: Not specified

Intervention type: Nursing

Intervention code: Bladder care

Search method: 3

Stage: Not specified

Disease: Not specified

Condition: Urinary retention

Intervention type: Not specified

Intervention code: Not specified

Search method: 4

Stage: Not specified

Disease: Not specified

Condition: Urinary Tract Infection

Intervention type: Not specified

Intervention code: Not specified

\section{Appendix 2. Search methods and terms used for the extra specific searching for earlier versions of this review (for Thomas 2005 and Thomas 2008)}

For earlier versions of this review (Thomas 2005; Thomas 2008) the following additional searches were conducted. Due to the comprehensive nature of the searches already performed by the Stroke Collaborative Review Group, no additional searches were performed other than on CINAHL, combining stroke terms with terms for urinary incontinence without a research methods filter. This was done because of the potentially poor indexing of nursing research. The following strategy was used to search CINAHL (January 1982 to January 2007) on Dialog Datastar: 
1. Urination-disorders\#.de

2. Urinary-tract-infections\#.de

3. Bladder-neurogenic.de

4. Bowel-and-bladder-management\#.de

5. Urologic-nursing.de

6. Urologic-care.de

7. Catheters-urinary\#.de

8. Catheter-care-urinary\#.de

9. Urinary-catheterization\#.de

10. Urinary-bladder-irrigation.de

11. Catheter-irrigation-urinary.de

12. Diagnosis-urologic\#.de

13. Urinary-tract-physiology\#.de

14. Incontinence-aids\#.de

15. Urinary-incontinence-and frequency-comfort-questionnaire.de

16. Tube-care-urinary-IOWA-NIC.de

17. Urinary-catheterisation-IOWA-NIC.de

18. Urinary-elimination-management-IOWA-NIC.de

19. Urinary-incontinence-care-IOWA-NIC.de

20. Urinary-retention-care-IOWA-NIC.de

21. Urinary-continence-IOWA-NOC.de

22. Urinary-elimination-IOWA-NOC.de

23. Altered-urinary-elimination-NANDA\#.de

24. Urinary-elimination-component-SABA-HHCC\#.de

25. Genito-urinary-function-OMAHA.de

26. Urin\$ or bladder or urethra\$).ti,ab.

27. Catheterization.w..de

28. Catheters.w..de

29. Catheter-care.de

30. Catheter-occlusion.de

31. Catheter-removal.de

32. Tube-removal.de

33. Catheter-placement-determination.de

34. Catheter-related-complications\#.de

35.26 and ( 27 or 28 or 29 or 30 or 31 or 32 or 33 or 34 )

36. Void $\$$ near (difficult $\$$ or impair $\$$ or problem\$ or disorder $\$$ or impair\$ or control\$)

37. 36.ti or 36.ab

38. Urin\$ with (incontinen\$ or continen\$)

39. 38.ti or 38.ab

40. Bladder with (incontin\$ or continen\$)

41. 40.ti or 40.ab

42. Detrusor with (instability or stability or stable or unstable)

43. 42. ti or 42.ab

44. Bladder with (instability or stability or stable or unstable)

45. 44.ti or 44.ab

46. (stress or urge or pad\$) with (continen\$ or incontinen\$)

47. 46.ti or 46.ab

48. Urodynamic $\$$.ti or urodynamic $\$$.ab

49.37 or 39 or 41 or 43 or 45 or 47 or 48

50.1 or 2 or 3 or 4 or 5 or 6 or 7 or 8 or 9 or 10 or 11 or 12 or 13 or 14 or 15 or 16 or 17 or 18 or 19 or 20 or 21 or 22 or 23 or 24 or 25

51.35 or 49 or 50

Key: \# = explode,. de = index word, \$ = wild card.

The above search was combined with the CINAHL search from the Cochrane Stroke Group.

Recent unpublished trial data were also searched for on national and international databases i.e. NHS National Research Register, NHS Research Findings Register, US Community of Science NIH Grants, MetaRegister of Controlled Clinical Trials and CRISP, by adapting terms drawn from the existing search strategies of the Incontinence and Stroke Review Groups.

The reference lists of all relevant reviews and trial reports were searched to identify further relevant studies. Major investigators were contacted to ask for any other possible relevant trials, published or unpublished. In addition, contact was made with the authors of 
other relevant Cochrane reviews to ascertain whether defined subgroups of stroke survivors were identified in trials testing methods of promoting continence in a general population.

The review was publicised on the following websites: Joanna Briggs Institute, Royal College of Nursing Research Society, Royal College of Nursing Continence Interest Group, Association of Continence Advisors, Sigma Theta Tau.

We did not impose any language or other restrictions on any of these searches.

\section{Appendix 3. Results of the search - previously published versions of this review}

\section{Results of the search for the 2008 review update}

The search for the 2008 review update (Thomas 2008) identified a further five studies (Liu 2006; Tibaek 2005; Zhang 2002; Zhou 1999). With the exception of Tibaek 2005, all were published in Chinese and were translated by a native speaking Chinese nursing student undertaking postgraduate study in England.

Eleven trials were therefore included in the 2008 review update. Five were full reports (Brittain 2000b; Chu 1997; Judge 1969; Liu 2006; Tibaek 2005), although one Chinese trial was only briefly described in a one page report (Chu 1997). The remaining five trials were reported only in conference abstracts (Gelber 1997a; Gelber 1997b; Lewis 1990; Zhang 2002; Zhou 1999).

\section{Results of the search for the original version of the review (2005)}

The original search for the first published version of this review identified 1234 papers and 126 studies were retrieved (Thomas 2005). The search initially identified nine potential trials evaluating the effectiveness of methods to promote continence in adults after stroke (Brittain 2000b; Chu 1997; Cook 1998; Gelber 1997a; Gelber 1997b; Gross 1990; Judge 1969; Lewis 1990; Tekeoglu 1998).

One trial was published in Chinese, one in Korean and the rest in English. Information from the Chinese trial was translated and extracted by nursing lecturers from the Shanghai Military Medical University. On further examination, three trials were excluded for reasons listed in the Characteristics of excluded studies (Cook 1998; Gross 1990; Tekeoglu 1998).

WHAT'S NEW

Date Event Description

29 January $2019 \quad$ New search has been performed

For this update (published in 2019):

1. The search was updated to November 2017 and a further 11 studies were identified. Two previously included trials were excluded as they were found to be ineligible. Therefore, the review contains 20 studies.

2. This version of the review has been substantively modified to comply with current Cochrane recommendations, including full risk of bias assessment, the implementation of GRADE to assess the quality of the body of evidence and the development of 'Summary of findings' tables. The title of the review has also been updated to reflect current Cochrane standards.

28 January $2019 \quad$ New citation required but conclusions have not changed
1. The review identified more trials of complementary therapy and transcutaneous electrical nerve stimulation: these warrant further investigation with the stroke population in adequately powered, multicentre trials.

2. There has been a change to the authorship.

\section{H I S T O R Y}

Protocol first published: Issue 4, 2003

Review first published: Issue 3, 2005 


\begin{tabular}{lll}
\hline Date & Event & Description \\
\hline 13 October 2008 & Amended & Converted to new review format. \\
\hline 14 November 2007 & $\begin{array}{l}\text { New citation required and conclusions } \\
\text { have changed }\end{array}$ & Substantive amendment. Five new studies added \\
\hline
\end{tabular}

\section{CONTRIBUTIONS OFAUTHORS}

LT: conceiving and designing the review, study screening, data extraction, assessment of risk of bias and writing of the review. LT acts as the guarantor of this review.

JC: study screening, data extraction, assessment of risk of bias and writing of the review.

LC: study screening, data extraction and assessment of risk of bias.

AT: translation of Chinese studies, interpretation of inclusion and exclusion criteria, data extraction and assessment of risk of bias.

CW: provided clinical input and contributed to constructing the review authors' conclusions.

\section{DECLARATIONSOF INTEREST}
LT: none known.
JC: none known.
LC: none known.
AT: none known.
CW: none known.

\section{SOURCES OF SUPPORT}

\section{Internal sources}

- Faculty of Health and Wellbeing, University of Central Lancashire, UK.

\section{External sources}

- National Institute of Health Research (NIHR), UK.

This project was supported by the National Institute for Health Research, via Cochrane Incentive Scheme and Infrastructure funding to Cochrane Incontinence. The views and opinions expressed therein are those of the authors and do not necessarily reflect those of the Systematic Reviews Programme, NIHR, National Health Service or the Department of Health. The NIHR is the largest single funder of Cochrane Incontinence.

\section{DIFFERENCES BETWEEN PROTOCOLANDREVIEW}

2019: This version of the review has been substantively modified to comply with current Cochrane recommendations, including full risk of bias assessment, the implementation of GRADE to assess the quality of the body of evidence and the development of 'Summary of findings' tables. The title of the review was updated to reflect current Cochrane standards. In addition, we made the following changes.

- The additional search of CINAHL was not updated, predominantly because both Specialised Register searches include CINAHL but also because this search did not yield additional studies for previous updates of the review.

- Additional searches of trials registries were not performed as the searches for both the Cochrane Stroke and Cochrane Incontinence Specialised Registers already cover a number of trials registries and platforms, including those specified in the Cochrane MECIR standards.

- Two previously included studies were excluded (Wikander 1998; Zhu 2003). Further inspection of these studies revealed participants were recruited within one month of stroke (acute phase), which is not covered by the scope of this review.

- Two secondary outcome measures originally listed in the protocol, economic outcomes and other outcomes, have now been removed.

- We have removed catheters and pads from the "physical aids" category of intervention; these are not designed to treat UI and catheters may be harmful. 


\section{N D EX TERMS}

\section{Medical Subject Headings (MeSH)}

Acupuncture Therapy [methods]; Complementary Therapies [methods]; Physical Therapy Modalities; Quality of Life; Randomized Controlled Trials as Topic; Stroke [ ${ }^{*}$ complications]; Stroke Rehabilitation; Urinary Incontinence [drug therapy] [etiology] [^therapy]

\section{MeSH check words}

Adult; Female; Humans; Male 